hep-th $/ 9811140$

SLAC-PUB-7992

UCLA $/ 98 / \mathrm{TEP} / 28$

UFIFT-HEP-98-33

November, 1998

\title{
Multi-Leg One-Loop Gravity Amplitudes from Gauge Theory
}

\author{
Z. Bern ${ }^{\star, 1}$, L. Dixon ${ }^{\dagger, 2}$, M. Perelstein ${ }^{\dagger, 2}$ and J.S. Rozowsky ${ }^{\ddagger, 3}$ \\ *Department of Physics, University of California at Los Angeles, Los Angeles, CA 90095-1547 \\ ${ }^{\dagger}$ Stanford Linear Accelerator Center, Stanford University, Stanford, CA 94309 \\ ${ }^{\ddagger}$ Institute for Fundamental Theory, Department of Physics, \\ University of Florida, Gainesville, FL 32611
}

\begin{abstract}
By exploiting relations between gravity and gauge theories, we present two infinite sequences of one-loop $n$-graviton scattering amplitudes: the 'maximally helicity-violating' amplitudes in $N=8$ supergravity, and the 'all-plus' helicity amplitudes in gravity with any minimally coupled massless matter content. The all-plus amplitudes correspond to self-dual field configurations and vanish in supersymmetric theories. We make use of the tree-level Kawai-Lewellen-Tye (KLT) relations between open and closed string theory amplitudes, which in the low-energy limit imply relations between gravity and gauge theory tree amplitudes. For $n \leq 6$, we determine the all-plus amplitudes explicitly from their unitarity cuts. The KLT relations, applied to the cuts, allow us to extend to gravity a previously found 'dimension-shifting' relation between (the cuts of) the all-plus amplitudes in gauge theory and the maximally helicity-violating amplitudes in $N=4$ super-Yang-Mills theory. The gravitational version of the relation lets us determine the $n \leq 6$ $N=8$ supergravity amplitudes from the all-plus gravity amplitudes. We infer the two series of amplitudes for all $n$ from their soft and collinear properties, which can also be derived from gauge theory using the KLT relations.
\end{abstract}

Submitted to Nuclear Physics B

\footnotetext{
${ }^{1}$ Research supported by the US Department of Energy under grant DE-FG03-91ER40662.

${ }^{2}$ Research supported by the US Department of Energy under grant DE-AC03-76SF00515.

${ }^{3}$ Research supported by the US Department of Energy under grants DE-FG03-91ER40662 and DEFG02-97ER41029.
} 


\section{Introduction}

Of the four known forces, three are well-described by gauge theories, and one by general relativity. While the quantum-mechanical properties of the former are reasonably well understood, the same cannot be said for the latter. In particular, gravity contains a dimensionful coupling parameter, $G_{N}=1 / M_{\text {Planck }}^{2}$, and therefore it is non-renormalizable at the level of power-counting. On the other hand, in perturbation theory certain relations have been found expressing gravity amplitudes in terms of gauge theory amplitudes. Such relations may lead to a better understanding of the properties of quantum gravity. At the classical (tree) level, Kawai, Lewellen and Tye (KLT) [1] found explicit general relations between closed- and open-string amplitudes. In the infinite-string-tension limit the KLT relations provide a representation of tree-level gravity amplitudes as the 'square' of tree-level gauge-theory amplitudes (where appropriate permutation sums and kinematic prefactors also have to be applied to the latter). These relationships, however, are not obvious from the point of view of the Lagrangian and the associated Feynman diagrams.

As an example of how such relations can lead to more explicit information about gravity amplitudes, one can consider special helicity assignments for the external gravitons and gauge bosons ('gluons'). The 'maximally helicity-violating' (MHV) configurations are those where exactly two particles have a helicity opposite to that of the remaining $n-2$ particles. (Tree amplitudes where all of the external particles have the same helicity, or just one has the opposite helicity, vanish trivially by a supersymmetry Ward identity (SWI) [2].) In gauge theory, the MHV tree amplitudes are known for all $n$, and are remarkably compact [3]. As we review in section 2, Berends, Giele and Kuijf (BGK) [4] used these gauge theory results together with the KLT relations to find a closed-form expression for the tree-level MHV gravity amplitudes. They also verified the universal behavior of these amplitudes as one of the graviton momenta becomes soft [5], providing a non-trivial consistency check on their results.

At the quantum level gravity should presumably be regarded as only an effective low-energy limit of some more fundamental theory, such as string or $M$ theory. It is nevertheless interesting to examine more carefully the quantum (loop) behavior of gravity, and its connection with gauge theory. The amplitudes that we investigate in this paper are ultraviolet finite in four dimensions, and should match the low-energy limit of any fundamental gravity theory.

The relation between gravity and gauge theory amplitudes has recently been exploited at the loop level for four-point amplitudes in the maximally supersymmetric versions of the two theories, namely $N=8$ supergravity and $N=4$ super-Yang-Mills theory [6]. It was shown that a squaring relation exists for the coefficients of various multi-loop integrals appearing in the two answers, similar to a relation found at one loop [7, 8]. This result was obtained for all terms in the two-loop amplitudes, and for some of the terms in higher-loop amplitudes (those that can be reconstructed entirely from two-particle unitarity cuts). The multi-loop relations have led to an improved understanding of the ultraviolet divergence structure of $N=8$ supergravity. In particular, at two loops the theory is finite for $D<7$, in contrast to a previous expectation of divergences beginning at $D=5$, which was based on an $N=4$ superspace power-counting [9]. Although the entire structure of the $N=8$ amplitudes is not yet known beyond two loops, the entirely-two-particle-constructible terms suggest 
that $N=8$ supergravity in $D=4$ is finite at three loops, and should only begin to diverge at five loops $[6]$; this is again in contrast to previous expectations $[10,7,9]$.

In this paper we continue the program of exploiting the relationship between gravity and gauge theory amplitudes to obtain non-trivial results for gravity. We use this relationship to obtain oneloop amplitudes with an arbitrary number $n$ of external gravitons. We consider two special types of helicity configurations: (1) the same MHV configurations that were considered at tree level by BGK, for the maximally supersymmetric theories of $N=8$ supergravity and $N=4$ super-YangMills; and (2) the 'all-plus' configurations where all the gravitons (or gauge bosons) have the same helicity, for non-supersymmetric theories with arbitrary massless matter content. (The all-plus amplitudes vanish for all supersymmetric theories). For gauge theory, both infinite sequences (1) and (2) are known [11, 12,13], and are reviewed in section 3. The all-plus gravity amplitudes have been presented in ref. [14]; here we provide details of the construction, as well as new results for the $N=8$ amplitudes.

These two sequences of gravity (gauge theory) amplitudes are not as different as one might expect, even though they involve different helicity states and different matter content. Indeed, in ref. [15] a 'dimension-shifting' relation was exhibited between the gauge theory amplitudes of types (1) and (2): The all-plus gauge amplitudes can be obtained from the $N=4 \mathrm{MHV}$ amplitudes by shifting the dimension of the loop integration upward by 4 units, $\int d^{D} L \rightarrow \int d^{D+4} L$, and multiplying by an overall prefactor. This relation was explicitly verified for $n=4,5,6$. In section 4.2 we use a combination of the KLT relations and unitarity to extend this relation from gauge theory to gravity, where it implies that the all-plus gravity amplitudes can be obtained from one-loop MHV $N=8$ supergravity amplitudes by shifting the dimension upward by 8 units, $\int d^{D} L \rightarrow \int d^{D+8} L$. (Ref. [15] also speculated on this relation for gravity, but only provided evidence for $n=4$.)

The dimension-shifting relation can be applied in either direction. Here we shall explicitly determine the all-plus $n$-graviton amplitudes for $n=4,5,6$, by calculating their unitarity cuts in an arbitrary dimension $D$ (see section 4 ). The cut calculations may be effectively performed by recycling the analogous gauge theory cut calculations. By working in an arbitrary dimension, we can determine the complete amplitudes from the cuts, free of the subtraction ambiguities frequently associated with dispersion relations [16]. Then (in section 4.4) we use the dimension-shifting relation of section 4.2 to obtain the MHV $N=8$ supergravity amplitudes. This provides a non-trivial example, beyond those in ref. [6], where gauge theory properties may be used to derive analogous results for gravity theories.

The one-loop MHV $N=4$ amplitudes can be written as linear combinations of a restricted class of scalar box (four-point) integrals. The one-loop MHV $N=8$ supergravity amplitudes can also be expressed in terms of the same class of box integrals. The relation between the coefficients of these integrals for the $N=8$ and $N=4$ cases is reminiscent of the tree-level KLT relations.

The unitarity-based results for gravity for $n \leq 6$ provide the starting point for constructing an ansatz for all $n$ which satisfies all known analytic properties. In order to go beyond the explicit graviton amplitude calculations for $n \leq 6$, we use the analytic properties of the two series of amplitudes as the $n$-point kinematics approaches special regions. In particular, we study the soft limits 
noted above, as well as the limits where two gravitons become collinear. Both of these limits for gravity can be understood at tree-level from the corresponding limits in gauge theory, by exploiting the KLT relations, as we show in section 5. We also show that there are no loop corrections to the structure of these limits in the case of gravity (unlike gauge theory). In section 6 we obtain ansatze that satisfy the appropriate limits for both the all-plus helicity (self-dual) gravity and $N=8$ supergravity series of amplitudes. Although we do not have a proof that the $n>6$ amplitudes are the unique expressions with the proper limits, we know of no counterexample with six- or higher-point kinematics where this method of obtaining amplitudes has failed to produce the correct expression.

The all-plus gravity and gauge amplitudes in $D=4$ are of interest in part because of their connection with self-dual gravity (SDG) [17] and self-dual Yang-Mills theory (SDYM) [18], i.e. gravity and gauge theory restricted to self-dual configurations of the respective field strengths, $R_{\mu \nu \rho \sigma}=\frac{i}{2} \epsilon_{\mu \nu}{ }^{\alpha \beta} R_{\alpha \beta \rho \sigma}$ and $F_{\mu \nu}=\frac{i}{2} \epsilon_{\mu \nu}{ }^{\alpha \beta} F_{\alpha \beta}$, with $\epsilon_{0123}=+1$. This connection is simple to see at the linearized (free) level of superpositions of plane waves of identical helicity. It has been further studied at tree level [19, 20, 21] and at the one-loop level [20, 22]. Chalmers and Siegel [22, 23] have presented self-dual actions for gravity and gauge theory which reproduce the all-plus scattering amplitudes at both tree level and one loop. Their actions have no amplitudes beyond one loop, and the tree-level amplitudes vanish on-shell. Thus the one-loop all-plus amplitudes constitute a complete perturbative solution to the theories defined by the Chalmers-Siegel self-dual actions. (See also ref. [14].)

In fact the one-loop gravity-gauge-theory relations can be stated in terms of just the components of the gauge amplitudes that dominate in the limit of a large number of colors, $N_{c}$. (These components are the $A_{n ; 1}$ partial amplitudes defined in section 3.) The large- $N_{c}$ limit of $N=4$ super-Yang-Mills theory has recently attracted much attention through its connection to superstring configurations in anti-de-Sitter space [24]. In this context, gauge theories with $N<4$ supersymmetry have been constructed by an orbifold-style [25] truncation of the $N=4$ spectrum, and it has been argued that at large- $N_{c}$ their amplitudes actually coincide (up to overall constants) with those of the $N=4$ theory [26]. These results may provide some additional motivation for studying the relation between large- $N_{c} N=4$ amplitudes and supergravity amplitudes, although we know of no direct connection between the purely perturbative relations found here and the anti-de-Sitter-space results, which are non-perturbative, involving a weak $\leftrightarrow$ strong coupling duality.

\section{Review of Tree-Level Properties}

In this section we review the tree-level KLT relations [1], and the known analytic properties of treelevel amplitudes in gauge theory and in gravity. These properties, as well as additional ones derived in section 5, will be used in section 6 to obtain an ansatz for the all-plus gravity and MHV $N=8$ supergravity amplitudes with an arbitrary number of external legs. 


\section{$2.1 \quad$ KLT Relations}

The KLT relations are between tree-level amplitudes in closed and open string theories, and arise from the representation of any closed string vertex operator as a product of open string vertex operators,

$$
V^{\text {closed }}\left(z_{i}, \bar{z}_{i}\right)=V_{\text {left }}^{\text {open }}\left(z_{i}\right) \bar{V}_{\text {right }}^{\text {open }}\left(\bar{z}_{i}\right) .
$$

The left and right string oscillators appearing in $V_{\text {left }}$ and $\bar{V}_{\text {right }}$ are distinct, but the zero mode momentum is shared. In the open string tree amplitude, the $z_{i}$ are real variables, to be integrated over the boundary of the disk, while in the closed string tree amplitude the $z_{i}$ are complex and integrated over the sphere. The closed-string integrand is thus a product of two open string integrands. This statement holds for any set of closed-string states, since they can all be written as tensor products of open-string states. KLT evaluated the $(n-3)$ two-dimensional closed-string world-sheet integrals, via a set of contour-integral deformations, in terms of the $(n-3)$ open-string integrals, and thereby related the two sets of string amplitudes.

After taking the field-theory limit [7, 8], $\alpha^{\prime} k_{i} \cdot k_{j} \rightarrow 0$, the KLT relations for four-, five- and six-point amplitudes are [4],

$$
\begin{gathered}
M_{4}^{\text {tree }}(1,2,3,4)=-i s_{12} A_{4}^{\text {tree }}(1,2,3,4) A_{4}^{\text {tree }}(1,2,4,3) \\
M_{5}^{\text {tree }}(1,2,3,4,5)=i s_{12} s_{34} A_{5}^{\text {tree }}(1,2,3,4,5) A_{5}^{\text {tree }}(2,1,4,3,5) \\
+i s_{13} s_{24} A_{5}^{\text {tree }}(1,3,2,4,5) A_{5}^{\text {tree }}(3,1,4,2,5), \\
M_{6}^{\text {tree }}(1,2,3,4,5,6)=-i s_{12} s_{45} A_{6}^{\text {tree }}(1,2,3,4,5,6)\left[s_{35} A_{6}^{\text {tree }}(2,1,5,3,4,6)\right. \\
\left.+\left(s_{34}+s_{35}\right) A_{6}^{\text {tree }}(2,1,5,4,3,6)\right] \\
+\mathcal{P}(2,3,4) .
\end{gathered}
$$

Here the $M_{n}$ 's are the amplitudes in a gravity theory stripped of couplings, the $A_{n}$ 's are the colorordered amplitudes in a gauge theory $[27,28], s_{i j} \equiv\left(k_{i}+k_{j}\right)^{2}$, and $\mathcal{P}(2,3,4)$ instructs one to sum over all permutations of the labels 2, 3 and 4 . The $n$ arguments of $M_{n}$ and $A_{n}$ are the external states $j$, which have momentum $k_{j}$. The $n$-point generalization of eq. $(2.2)[1,4]$ is presented in appendix A.

Each gravity state $j$ appearing in $M_{n}$ is the tensor product of the corresponding two gauge theory states appearing in the $A_{n}$ 's on the right-hand side of the equation. In particular, each of the 256 states of $N=8$ supergravity multiplet, consisting of 1 graviton, 8 gravitinos, 28 gauge bosons, 56 gauginos, and 70 real scalars, can be interpreted as a tensor product of two sets of the 16 states of the $N=4$ super-Yang-Mills multiplet, consisting of 1 gluon, 4 gluinos and 6 real scalars. (In string theory, this correspondence may be understood in terms of the factorization of the closed string vertex operator for each $N=8$ state into a product of $N=4$ open string vertex operators.) Thus a sum over the $N=8$ supergravity states can be interpreted as a double sum over a tensor product of $N=4$ super-Yang-Mills states. 
Full amplitudes are obtained from $M_{n}^{\text {tree }}$ and $A_{n}^{\text {tree }}$ via,

$$
\begin{aligned}
\mathcal{M}_{n}^{\text {tree }}(1,2, \ldots, n) & =\left(\frac{\kappa}{2}\right)^{(n-2)} M_{n}^{\text {tree }}(1,2, \ldots, n), \\
\mathcal{A}_{n}^{\text {tree }}(1,2, \ldots, n) & =g^{(n-2)} \sum_{\sigma \in S_{n} / Z_{n}} \operatorname{Tr}\left(T^{a_{\sigma(1)}} T^{a_{\sigma(2)}} \cdots T^{a_{\sigma(n)}}\right) A_{n}^{\text {tree }}(\sigma(1), \sigma(2), \ldots, \sigma(n)),
\end{aligned}
$$

where $\kappa^{2}=32 \pi G_{N}$, and $S_{n} / Z_{n}$ is the set of all permutations, but with cyclic rotations removed. The $T^{a_{i}}$ are fundamental representation matrices for the Yang-Mills gauge group $S U\left(N_{c}\right)$, normalized so that $\operatorname{Tr}\left(T^{a} T^{b}\right)=\delta^{a b}$.

The relations (2.2) hold for arbitrary external states. For external gravitons and gluons it is convenient to quote the results in a helicity basis, using the spinor helicity formalism [29]. At treelevel - and to all orders for supersymmetric theories - helicity amplitudes where all, or all but one, of the external particles have the same helicity vanish by a SWI [2],

$$
M_{n}( \pm,+,+, \ldots,+)=A_{n}( \pm,+,+, \ldots,+)=0
$$

where the helicity assignments are for outgoing particles. These relations hold for any states in the respective $N=8$ and $N=4$ multiplets. (For scalar states, one interprets 'helicity' as particle vs. anti-particle.)

For a given number of external legs $n$, the simplest non-vanishing tree amplitudes — and supersymmetric loop amplitudes - are the maximally helicity-violating (MHV) amplitudes, where exactly two helicities are opposite to the majority. At tree-level, and to all loop orders in $N=4$ super-Yang-Mills theory, the MHV $n$-gluon amplitudes are all related to each other by the $N=4$ SWI [15],

$$
\frac{1}{\langle i j\rangle^{4}} \mathcal{A}_{n}\left(1^{+}, 2^{+}, \ldots, i^{-}, \ldots, j^{-}, \ldots, n^{+}\right)=\frac{1}{\langle a b\rangle^{4}} \mathcal{A}_{n}\left(1^{+}, 2^{+}, \ldots, a^{-}, \ldots, b^{-}, \ldots, n^{+}\right),
$$

where $i$ and $j$ are the only negative helicity legs on the left-hand side and $a$ and $b$ are the only negative helicities on the right-hand side. (We will generally indicate the type of external particle by a subscript; e.g. $1_{g}^{-}$for a negative-helicity gluon. However, for gluons in gauge theory and gravitons in gravity, we will usually omit the subscript.) Thus at tree-level it suffices to give the formula [3]

$$
A_{n}^{\text {tree }}\left(1^{-}, 2^{-}, 3^{+}, 4^{+}, \ldots, n^{+}\right)=i \frac{\langle 12\rangle^{4}}{\langle 12\rangle\langle 23\rangle\langle 34\rangle \cdots\langle n 1\rangle} .
$$

We use the notation $\left\langle k_{i}^{-} \mid k_{j}^{+}\right\rangle=\langle i j\rangle$ and $\left\langle k_{i}^{+} \mid k_{j}^{-}\right\rangle=[i j]$, where $\left|k_{i}^{ \pm}\right\rangle$are massless Weyl spinors, labeled with the sign of the helicity and normalized by $\langle i j\rangle[j i]=s_{i j}=2 k_{i} \cdot k_{j}$. For the case where both energies are positive the spinor inner products are given by

$$
\langle i j\rangle=\sqrt{\left|s_{i j}\right|} e^{i \phi_{i j}}, \quad[i j]=\sqrt{\left|s_{i j}\right|} e^{-i\left(\phi_{i j}+\pi\right)},
$$

where

$$
\cos \phi_{i j}=\frac{k_{i}^{1} k_{j}^{+}-k_{j}^{1} k_{i}^{+}}{\sqrt{\left|s_{i j}\right| k_{i}^{+} k_{j}^{+}}}, \quad \quad \sin \phi_{i j}=\frac{k_{i}^{2} k_{j}^{+}-k_{j}^{2} k_{i}^{+}}{\sqrt{\left|s_{i j}\right| k_{i}^{+} k_{j}^{+}}}
$$


and $k_{i}^{+}=k_{i}^{0}+k_{i}^{3}$. (The cases where one or both of the energies are negative are similar, except for additional overall phases.) For later use, we also define the spinor strings

$$
\begin{aligned}
\left\langle i^{-}|(l+m)| j^{-}\right\rangle & \equiv\left\langle k_{i}^{-}\left|\left(k_{l}+\not k_{m}\right)\right| k_{j}^{-}\right\rangle, \\
\left\langle i^{+}|l m \cdots| j^{-}\right\rangle & \equiv\left\langle k_{i}^{+}\left|\not k_{l} k_{m} \cdots\right| k_{j}^{-}\right\rangle, \\
\left\langle i^{-}\left|\ell_{m}\right| j^{-}\right\rangle & \equiv\left\langle k_{i}^{-}\left|\ell_{m}\right| k_{j}^{-}\right\rangle,
\end{aligned}
$$

etc., where $\ell_{m}$ is a loop momentum.

The MHV graviton tree (and $N=8$ loop) amplitudes satisfy an $N=8$ SWI analogous to eq. (2.5),

$$
\frac{1}{\langle i j\rangle^{8}} \mathcal{M}_{n}\left(1^{+}, 2^{+}, \ldots, i^{-}, \ldots, j^{-}, \ldots, n^{+}\right)=\frac{1}{\langle a b\rangle^{8}} \mathcal{M}_{n}\left(1^{+}, 2^{+}, \ldots, a^{-}, \ldots, b^{-}, \ldots, n^{+}\right) .
$$

The MHV four- and five-graviton tree amplitudes, ${ }^{1}$ which satisfy eq. (2.10) as well as the appropriate KLT relations $(2.2)$, are [4]

$$
\begin{aligned}
M_{4}^{\text {tree }}\left(1^{-}, 2^{-}, 3^{+}, 4^{+}\right) & =i\langle 12\rangle^{8} \frac{[12]}{\langle 34\rangle N(4)}, \\
M_{5}^{\text {tree }}\left(1^{-}, 2^{-}, 3^{+}, 4^{+}, 5^{+}\right) & =i\langle 12\rangle^{8} \frac{\varepsilon(1,2,3,4)}{N(5)},
\end{aligned}
$$

where

$$
\varepsilon(i, j, m, n) \equiv 4 i \varepsilon_{\mu \nu \rho \sigma} k_{i}^{\mu} k_{j}^{\nu} k_{m}^{\rho} k_{n}^{\sigma}=[i j]\langle j m\rangle[m n]\langle n i\rangle-\langle i j\rangle[j m]\langle m n\rangle[n i],
$$

and

$$
N(n) \equiv \prod_{i=1}^{n-1} \prod_{j=i+1}^{n}\langle i j\rangle .
$$

For $n>4$, Berends, Giele and Kuijf [4] presented the expression,

$$
\begin{aligned}
M_{n}^{\text {tree }}\left(1^{-}\right. & \left., 2^{-}, 3^{+}, \ldots, n^{+}\right)=-i\langle 12\rangle^{8} \\
& \times\left[\frac{[12][n-2 n-1]}{\langle 1 n-1\rangle N(n)}\left(\prod_{i=1}^{n-3} \prod_{j=i+2}^{n-1}\langle i j\rangle\right) \prod_{l=3}^{n-3}\left(-\left\langle n^{-}\left|K_{l+1, n-1}\right| l^{-}\right\rangle\right)+\mathcal{P}(2,3, \ldots, n-2)\right],
\end{aligned}
$$

where $K_{i, j}^{\mu} \equiv \sum_{s=i}^{j} k_{s}^{\mu}$, and $+\mathcal{P}(M)$ instructs one to sum the quantity inside the brackets over all permutations of the set $M$. They numerically verified its correctness for $n \leq 11$. The expression in brackets is totally symmetric (although this is not manifest), as is required for consistency with eq. (2.10).

\subsection{Soft and Collinear Properties at Tree Level}

There are two important universal limits of color-ordered $n$-gluon tree amplitudes. In the limit that the gluon $s$ becomes soft, $A_{n}^{\text {tree }}$ has the universal behavior [30],

$$
A_{n}^{\text {tree }}\left(\ldots, a, s^{ \pm}, b, \ldots\right) \stackrel{k_{s} \rightarrow 0}{\longrightarrow} \mathcal{S}^{\text {tree }}\left(a, s^{ \pm}, b\right) \times A_{n-1}^{\text {tree }}(\ldots, a, b, \ldots),
$$

\footnotetext{
${ }^{1}$ Our overall phase conventions differ from those of ref. [4] by a ' $-i$ '.
} 
where the soft (eikonal) factors are

$$
\begin{aligned}
\mathcal{S}^{\text {tree }}\left(a, s^{+}, b\right) & =\frac{\langle a b\rangle}{\langle a s\rangle\langle s b\rangle}, \\
\mathcal{S}^{\text {tree }}\left(a, s^{-}, b\right) & =\frac{-[a b]}{[a s][s b]} .
\end{aligned}
$$

In the collinear limit where two gluon momenta $k_{a}$ and $k_{b}$ become parallel (denoted by $a \| b$ ), we have $k_{a} \approx z k_{P}$ and $k_{b} \approx(1-z) k_{P}$ for some $z \in[0,1]$, where $k_{P} \equiv k_{a}+k_{b}$. The behavior of a tree amplitude in this limit is [31]

$$
A_{n}^{\text {tree }}\left(\ldots, a^{\lambda_{a}}, b^{\lambda_{b}}, \ldots\right) \stackrel{a \| b}{\longrightarrow} \sum_{\lambda= \pm} \operatorname{Split}_{-\lambda}^{\text {tree }}\left(z, a^{\lambda_{a}}, b^{\lambda_{b}}\right) \times A_{n-1}^{\text {tree }}\left(\ldots, P^{\lambda}, \ldots\right)
$$

where the gluon splitting amplitudes are

$$
\begin{aligned}
& \operatorname{Split}_{+}^{\text {tree }}\left(z, a^{+}, b^{+}\right)=0, \\
& \operatorname{Split}_{-}^{\text {tree }}\left(z, a^{+}, b^{+}\right)=\frac{1}{\sqrt{z(1-z)}\langle a b\rangle}, \\
& \operatorname{Split}_{+}^{\text {tree }}\left(z, a^{-}, b^{+}\right)=\sqrt{\frac{z^{3}}{1-z}} \frac{1}{\langle a b\rangle} ;
\end{aligned}
$$

the remaining ones may be obtained by parity. Similar expressions exist including fermions. For example, for a gluon splitting into two fermions, the color-ordered splitting amplitudes are

$$
\operatorname{Split}_{+}^{\text {tree }}\left(z, a_{\bar{q}}^{-}, b_{q}^{+}\right)=\frac{z}{\langle a b\rangle}, \quad \quad \operatorname{Split}_{-}^{\text {tree }}\left(z, a_{\bar{q}}^{-}, b_{q}^{+}\right)=\frac{1-z}{[a b]} .
$$

A more complete discussion of splitting amplitudes may be found in reviews [31, 32, 16]. (Our sign conventions in the splitting functions are the ones used in ref. [33].)

Gravity amplitudes, like gauge amplitudes, are known to satisfy universal soft limits[5, 4]. The gravitational soft limits have the form,

$$
M_{n}^{\text {tree }}\left(\ldots, a, s^{ \pm}, b, \ldots\right) \stackrel{k_{s} \rightarrow 0}{\longrightarrow} \mathcal{S}^{\text {gravity }}\left(s^{ \pm}\right) \times M_{n-1}^{\text {tree }}(\ldots, a, b, \ldots) .
$$

For the limit $k_{n} \rightarrow 0$ in $M_{n}^{\text {tree }}(1,2, \ldots, n)$, the gravitational soft factor (for positive helicity) is

$$
\mathcal{S}_{n} \equiv \mathcal{S}^{\text {gravity }}\left(n^{+}\right)=\frac{-1}{\langle 1 n\rangle\langle n, n-1\rangle} \sum_{i=2}^{n-2} \frac{\langle 1 i\rangle\langle i, n-1\rangle[i n]}{\langle i n\rangle} .
$$

Although it is not manifest, $\mathcal{S}_{n}$ is also symmetric under the interchange of legs 1 and $n-1$ with the others. For all $n$, BGK verified that the MHV amplitudes (2.14) have the correct behavior as an external momentum becomes soft.

In section 5 we will show that gravity tree amplitudes also have universal behavior as two external momenta become collinear, and that the splitting amplitudes are composed of products of pairs of the ones for gauge theory. We shall further demonstrate that the tree-level soft factors and splitting amplitudes for gravity do not incur any higher loop corrections, in contrast to the situation for gauge theory. 


\section{One-Loop MHV Amplitudes in Gauge Theory}

Before proceeding to gravity, it is useful to review the structure of the one-loop maximally helicityviolating (MHV) amplitudes in gauge theory $[12,13,11,34]$. These amplitudes were constructed by techniques similar to those used in the following sections for the corresponding gravity amplitudes. In particular we shall use unitarity or cutting techniques $[35,11,34,36]$, as well as the factorization bootstrap approach $[3,12]$ of finding ansatze for amplitudes based on their known kinematic poles. In appendix B we outline an alternative approach to the all-plus gravity amplitudes (as well as to various tree-level gravity amplitudes), one based on recursive techniques [37, 38, 13].

The unitarity cuts in the $N=4 \mathrm{MHV}$ gauge case are simple enough that the direct computation can be performed for all $n$ simultaneously. We have not been able to do that yet for the analogous $N=8 \mathrm{MHV}$ supergravity computation, and so we shall resort to an ansatz for $n>6$, based on soft and collinear limits.

\subsection{General Properties of One-Loop Amplitudes}

We first define one-loop amplitudes $M_{n}$ for gravity and $A_{n ; j}$ gauge theory, from which all couplings have been removed. Color has also been removed from the $A_{n ; j}$, according to the one-loop color decomposition [39]. For the case where all states are in the adjoint representation, the full amplitudes are given by,

$$
\begin{aligned}
\mathcal{M}_{n}^{1 \text { loop }}(1,2, \ldots, n) & =\left(\frac{\kappa}{2}\right)^{n} M_{n}(1,2, \ldots, n), \\
\mathcal{A}_{n}^{1 \text {-loop }}(1,2, \ldots, n) & =g^{n} \sum_{j=1}^{\lfloor n / 2\rfloor+1} \sum_{\sigma \in S_{n} / S_{n ; j}} \operatorname{Gr}_{n ; j}(\sigma) A_{n ; j}(\sigma(1), \ldots, \sigma(n)),
\end{aligned}
$$

where $\lfloor x\rfloor$ denotes the greatest integer less than or equal to $x$, the (unpermuted) color trace structures are $\mathrm{Gr}_{n ; 1}(1) \equiv N_{c} \operatorname{Tr}\left(T^{a_{1}} \cdots T^{a_{n}}\right)$ and $\mathrm{Gr}_{n ; j}(1)=\operatorname{Tr}\left(T^{a_{1}} \cdots T^{a_{j-1}}\right) \operatorname{Tr}\left(T^{a_{j}} \cdots T^{a_{n}}\right)$ for $j>1$, and $S_{n ; j}$ is the subset of permutations $S_{n}$ that leaves the trace structure $\mathrm{Gr}_{n ; j}$ invariant. Similar color decompositions exist for the cases with fundamental representation particles in the loop. In fact, the partial amplitudes $A_{n ; j}$ for $j>1$ can be expressed in terms of the $A_{n ; 1}$ through the formula [11],

$$
A_{n ; j}(1,2, \ldots, j-1 ; j, j+1, \ldots, n)=(-1)^{j-1} \sum_{\sigma \in C O P\{\alpha\}\{\beta\}} A_{n ; 1}(\sigma) .
$$

Here $\alpha_{i} \in\{\alpha\} \equiv\{j-1, j-2, \ldots, 2,1\}, \beta_{i} \in\{\beta\} \equiv\{j, j+1, \ldots, n-1, n\}$, and $C O P\{\alpha\}\{\beta\}$ is the set of all permutations of $\{1,2, \ldots, n\}$ with $n$ held fixed that preserve the cyclic ordering of the $\alpha_{i}$ within $\{\alpha\}$ and of the $\beta_{i}$ within $\{\beta\}$, while allowing for all possible relative orderings of the $\alpha_{i}$ with respect to the $\beta_{i}$.

Thus the full gauge amplitude can be constructed just from the $A_{n ; 1}$, which are color-ordered (i.e, they only receive contributions from planar graphs with a fixed ordering of the external legs), and therefore have simpler analytic properties than the remaining $A_{n ; j}$. For this reason we need

only explicitly discuss the case of $A_{n ; 1}$. The $A_{n ; 1}$ contributions are the ones which dominate the amplitude for a large number of colors $N_{c}$. 
We consider one-loop amplitudes where the external momenta are taken to lie in four dimensions, but the number of dimensions $D$ appearing in the loop-momentum integration measure $d^{D} L$ remains arbitrary (for the time being). (To maintain supersymmetry we leave the number of states at their four-dimensional values.) In general, the $m$-point loop integrals with $m \geq 5$ which appear in such one-loop amplitudes can be reduced down to at most box (four-point) integrals and pentagon (fivepoint) integrals, where the pentagon integrals are scalar integrals (i.e., they contain no loop momenta in the numerator of the integrand) and are evaluated in $D+2$ dimensions [40]. Furthermore, if we now set $D=4-2 \epsilon$, then to $\mathcal{O}\left(\epsilon^{0}\right)$ the pentagon contributions may be neglected, because the scalar pentagon integrals in $D=6-2 \epsilon$ have no poles as $\epsilon \rightarrow 0$ (they are infrared and ultraviolet finite in $D=6$ ), and because they are generated in the integral reduction procedure with a manifest $\epsilon$ prefactor [40].

For an amplitude in a generic theory, after applying these reductions the box integrals may have powers of the loop momentum $L$ inserted in the numerator of the integrand, in addition to the four scalar propagators which make up the denominator. The amplitude may also contain triangle and bubble integrals arising from the corresponding Feynman diagrams. The all-plus helicity and $N=4$ supersymmetric cases which we discuss below are special, however, and do not contain the full set of possible scalar integrals.

\subsection{Review of One-Loop Soft and Collinear Properties in Gauge Theory}

The collinear limits for the leading color-ordered one-loop amplitudes, $A_{n ; 1}$, are similar to the treelevel case and have the form

$$
\begin{aligned}
A_{n ; 1}\left(\ldots, a^{\lambda_{a}}, b^{\lambda_{b}}, \ldots\right) \stackrel{a \| b}{\longrightarrow} \sum_{\lambda= \pm}( & \operatorname{Split}_{-\lambda}^{\text {tree }}\left(z, a^{\lambda_{a}}, b^{\lambda_{b}}\right) A_{n-1 ; 1}\left(\ldots, P^{\lambda}, \ldots\right) \\
& \left.+\operatorname{Split}_{-\lambda}^{1-\text { loop }}\left(z, a^{\lambda_{a}}, b^{\lambda_{b}}\right) A_{n-1}^{\text {tree }}\left(\ldots, P^{\lambda}, \ldots\right)\right) .
\end{aligned}
$$

In addition to the tree-level splitting amplitudes, $\operatorname{Split}_{-\lambda}^{\mathrm{tree}}\left(z, a^{\lambda_{a}}, b^{\lambda_{b}}\right)$, one-loop corrections now also appear, $\mathrm{Split}_{-\lambda}^{1-\mathrm{loop}}\left(z, a^{\lambda_{a}}, b^{\lambda_{b}}\right)$. Both quantities are universal, depending only on the two momenta becoming collinear, and not upon the specific amplitude under consideration [41]. The explicit values of the $\operatorname{Split}_{-\lambda}^{1-\text { loop }}\left(z, a^{\lambda_{a}}, b^{\lambda_{b}}\right)$ (which we shall not need here) were originally determined [11] from the four- [42] and five-point [43,33] one-loop gauge amplitudes. Their universality for an arbitrary number of external legs was demonstrated in ref. [41].

Similarly, as the momentum of an external leg becomes soft the color-ordered one-loop amplitudes behave as,

$$
\begin{aligned}
A_{n ; 1}\left(\ldots, a, s^{ \pm}, b, \ldots\right) \stackrel{k_{s \rightarrow 0} \rightarrow}{\longrightarrow} \mathcal{S}^{\text {tree }}( & \left(a, s^{ \pm}, b\right) A_{n-1 ; 1}(\ldots, a, b, \ldots) \\
& +\mathcal{S}^{1-\operatorname{loop}}\left(a, s^{ \pm}, b\right) A_{n-1}^{\text {tree }}(\ldots, a, b, \ldots),
\end{aligned}
$$

where $\mathcal{S}^{1 \text {-loop }}\left(a, s^{ \pm}, b\right)$ is universal.

In the application of eqs. (3.3) and (3.4) to the collinear and soft limits of the one-loop allplus gauge amplitudes, the second term always drops out, because of the vanishing of the tree-level 
amplitudes with all plus helicities, or all but one plus. In the $N=4$ super-Yang-Mills MHV case, however, the contributions of $\operatorname{Split}_{-\lambda}^{1-\text { loop }}\left(z, a^{\lambda_{a}}, b^{\lambda_{b}}\right)$ and $\mathcal{S}^{1-\text { loop }}\left(a, s^{ \pm}, b\right)$ survive.

\subsection{All-Plus Amplitudes}

The analytic properties of the one-loop all-plus amplitudes in gauge theory $[12,13]$ are remarkably simple. First of all, the unitarity cuts vanish in four dimensions, since eq. (2.4) implies that at least one of the two tree amplitudes on either side of a unitarity cut vanishes, for every possible helicity assignment for the two gluons crossing the cut. Similarly, by considering their factorization on particle poles, one finds that the one-loop all-plus amplitudes cannot contain multi-particle poles, i.e., factors of the form $1 /\left(k_{i_{1}}+k_{i_{2}}+\cdots+k_{i_{m}}\right)^{2}$ with $m>2$. The only permitted kinematic singularities are the ones where one external momentum becomes soft, or two external momenta become collinear. Finally, the loop-momentum integration does not generate any infrared nor ultraviolet divergences or associated logarithms. In summary, $A_{n ; 1}\left(1^{+}, 2^{+}, \ldots, n^{+}\right)$is a finite rational function of the momenta, cyclically symmetric in its $n$ arguments, with singularities only in the regions where a momentum is soft or two cyclically (color) adjacent momenta are collinear.

These analytic properties were crucial in obtaining an ansatz for the explicit form of the amplitudes [12], which was verified by Mahlon via recursive techniques [13]. The one-loop amplitudes for $n$ identical-helicity gluons in pure Yang-Mills theory are,

$$
A_{n ; 1}\left(1^{+}, 2^{+}, \ldots, n^{+}\right)=-\frac{i}{48 \pi^{2}} \sum_{1 \leq i_{1}<i_{2}<i_{3}<i_{4} \leq n} \frac{\operatorname{tr}_{-}\left[i_{1} i_{2} i_{3} i_{4}\right]}{\langle 12\rangle\langle 23\rangle \cdots\langle n 1\rangle},
$$

where $\operatorname{tr}_{ \pm}\left[i_{1} i_{2} i_{3} i_{4}\right] \equiv \frac{1}{2} \operatorname{tr}\left[\left(1 \pm \gamma_{5}\right) \not k_{i_{1}} \not k_{i_{2}} \not k_{i_{3}} \not k_{i_{4}}\right]$. These amplitudes are generated by actions for selfdual Yang-Mills theory [20, 22] as well as ordinary gauge theory.

The all-plus amplitudes vanish in any supersymmetric theory by the SWI (2.4). Thus the contribution of a gluon circulating around the loop is the negative of that from an adjoint fermion in the loop, and equal to that from an adjoint scalar. For a fundamental representation fermion one must divide the contribution to $A_{n ; 1}$ by an additional factor of $N_{c}$. In particular, for QCD with $n_{f}$ flavors of quarks we have

$$
A_{n ; 1}^{\mathrm{QCD}}\left(1^{+}, 2^{+}, \ldots, n^{+}\right)=\left(1-\frac{n_{f}}{N_{c}}\right) A_{n ; 1}\left(1^{+}, 2^{+}, \ldots, n^{+}\right) .
$$

For $n \leq 6$, the all-plus amplitudes have also been computed to all orders in the dimensional regularization parameter $\epsilon$ (but with four-dimensional external momenta) via their unitarity cuts [15]. 
These amplitudes may be compactly expressed as

$$
\begin{aligned}
A_{4 ; 1}\left(1^{+}, 2^{+}, 3^{+}, 4^{+}\right)= & \frac{-2 s_{12} s_{23}}{\langle 12\rangle\langle 23\rangle\langle 34\rangle\langle 41\rangle} \mathcal{I}_{4}^{1234}\left[\mu^{4}\right], \\
A_{5 ; 1}\left(1^{+}, 2^{+}, 3^{+}, 4^{+}, 5^{+}\right)= & \frac{1}{\langle 12\rangle\langle 23\rangle\langle 34\rangle\langle 45\rangle\langle 51\rangle}\left\{\left[-s_{12} s_{23} \mathcal{I}_{4}^{123(45)}\left[\mu^{4}\right]+\text { cyclic }\right]\right. \\
& \left.+2 \varepsilon(1,2,3,4) \mathcal{I}_{5}^{12345}\left[\mu^{6}\right]\right\}, \\
A_{6 ; 1}\left(1^{+}, 2^{+}, 3^{+}, 4^{+}, 5^{+}, 6^{+}\right)= & \frac{1}{\langle 12\rangle\langle 23\rangle\langle 34\rangle\langle 45\rangle\langle 56\rangle\langle 61\rangle}\left\{\left[-s_{12} s_{23} \mathcal{I}_{4}^{123(456)}\left[\mu^{4}\right]\right.\right. \\
& \left.-\frac{1}{2}\left(t_{123} t_{234}-s_{23} s_{56}\right) \mathcal{I}_{4}^{1(23) 4(56)}\left[\mu^{4}\right]+\varepsilon(1,2,3,4) \mathcal{I}_{5}^{1234(56)}\left[\mu^{6}\right]+\text { cyclic }\right] \\
& \left.-\operatorname{tr}[123456] \mathcal{I}_{6}^{123456}\left[\mu^{6}\right]\right\},
\end{aligned}
$$

where $s_{i j}=\left(k_{i}+k_{j}\right)^{2}, t_{i j l}=\left(k_{i}+k_{j}+k_{l}\right)^{2}$, and ' + cyclic' implies a sum over the $n$ cyclic permutations (for $A_{n ; 1}$ ) of the quantity within the brackets ([ ]) in which the phrase appears.

In eq. (3.7), $\mathcal{I}_{5}^{12345}$ and $\mathcal{I}_{6}^{123456}$ are pentagon and hexagon integrals where all the external legs are massless. The integral $\mathcal{I}_{5}^{1234(56)}$ is a one-mass pentagon integral, where legs 5 and 6 form the one external mass. The parentheses in the arguments of the one- and two-mass box integrals $\mathcal{I}_{4}$ similarly indicate the grouping of massless external legs for the amplitude into massive legs for the integral. (See appendix D for further exposition of our notation for the integrals.) In the integration measure $d^{D} L$ for the integrals, the $(D=4-2 \epsilon)$-dimensional loop momentum $L$ can be decomposed into a 4-dimensional part $\ell$ and a $(-2 \epsilon)$-dimensional part $\mu$, as $L=\ell+\mu$. Following the prescriptions of 't Hooft and Veltman [44], we take the four- and $(-2 \epsilon)$-dimensional parts of the loop momenta to be orthogonal, so that $L^{2}=\ell^{2}-\mu^{2}$. The symbol ' $\left[\mu^{2 r}\right]$ ' instructs one to insert an extra factor of $\mu^{2 r} \equiv\left(\mu^{2}\right)^{r}$ into the loop integrand before performing the integral, i.e.,

$$
\mathcal{I}_{m}\left[\mu^{2 r}\right] \equiv \int \frac{d^{4} \ell}{(2 \pi)^{4}} \frac{d^{-2 \epsilon} \mu}{(2 \pi)^{-2 \epsilon}} \frac{\left(\mu^{2}\right)^{r}}{\left.\left(\ell^{2}-\mu^{2}\right)\left(\left(\ell-K_{1}\right)^{2}-\mu^{2}\right) \ldots\left(\ell-\sum_{i=1}^{m-1} K_{i}\right)^{2}-\mu^{2}\right)} .
$$

Carrying out the $\mu$ integration explicitly leads to the formula [13, 36, 15]

$$
\mathcal{I}_{m}\left[\mu^{2 r}\right]=-\epsilon(1-\epsilon) \cdots(r-1-\epsilon)(4 \pi)^{r} \mathcal{I}_{m}^{D=4+2 r-2 \epsilon},
$$

where $\mathcal{I}_{m}^{D=4+2 r-2 \epsilon}$ is $\mathcal{I}_{m}$ with the number of dimensions in the loop-momentum integration shifted upward by $2 r$; i.e., one replaces $D \rightarrow D+2 r$ in eq. (D.1).

The fact that the all-orders-in- $\epsilon$ formulas (3.7) for the all-plus amplitudes contain insertions of the $(-2 \epsilon)$-dimensional components of the loop-momentum is just a reflection of the vanishing of the amplitudes' unitarity cuts for $D=4(\epsilon \rightarrow 0)$. It is straightforward to show that eq. (3.7) reduces to the $n \leq 6$ cases of eq. (3.5) as $\epsilon \rightarrow 0$. The explicit $\epsilon$ in the prefactor of $\mathcal{I}_{m}^{D=4+2 r-2 \epsilon}$ in eq. (3.9) means that only the $1 / \epsilon$ pole coming from the ultraviolet divergence of $\mathcal{I}_{m}^{D=4+2 r-2 \epsilon}$ will contribute. These contributions, which are pure numbers, are given in appendix D. 


\section{4 $N=4$ Super Yang-Mills Amplitudes}

The one-loop MHV amplitudes of $N=4$ super-Yang-Mills theory provide another example of amplitudes that may be evaluated for an arbitrary number of external legs. The higher degree of supersymmetry present in $N=4$ super-Yang-Mills theory considerably simplifies the analytic properties of its loop amplitudes. (Infinite sequences of MHV amplitudes have also been determined for $N=1$ supersymmetric theories, but their analytic structure is more complicated [34].)

In particular, supersymmetry cancellations forbid all triangle and bubble integrals, and only scalar box integrals (with no loop momenta in the numerator) may appear [11]. These supersymmetry cancellations, which may be seen in $N=1$ superspace [45], or in components by using a string-based approach [46,47], imply a maximum of $m-4$ powers of loop momentum in the numerator of an $m$-point integral. The integral reduction procedure mentioned in section 3.1 uses equations such as $L \cdot k_{i}=-\frac{1}{2}\left(\left(L-k_{i}\right)^{2}-L^{2}-k_{i}^{2}\right)$, where $L$ is the loop momentum and $k_{i}$ is an external momentum. The factors $L^{2}$ and $\left(L-k_{i}\right)^{2}$ cancel denominator factors from scalar propagators and reduce the number of external legs for the integral by one [48]. Thus the degree of the loop-momentum polynomial in the numerator of the integral is reduced by one whenever the number of legs for the integral is reduced by one. As a consequence, the $m$-point integrals in $N=4$ superYang-Mills theory can lead, after reduction, to at most scalar box integrals. Later, in section 4.5, we shall compare this loop-momentum power counting to what we find from inspecting the $N=8$ MHV supergravity amplitudes.

Although the $N=4$ power-counting allows any scalar box integral to appear at one loop, for the MHV helicity configurations one finds only the two-mass scalar box integrals where the two massive legs are diagonally opposite [11]. (Massless legs of the integral correspond directly to external momenta of the amplitude, while massive legs correspond to sums of external momenta.) Denoting the massless legs by $a$ and $b$, we define

$$
\mathcal{I}_{4}^{a K_{1} b K_{2}} \equiv \mathcal{I}_{4}^{a(a+1, \ldots, b-1) b(b+1, \ldots, a-1)}=\int \frac{d^{D} L}{(2 \pi)^{D}} \frac{1}{L^{2}\left(L-k_{a}\right)^{2}\left(L-k_{a}-K_{1}\right)^{2}\left(L+K_{2}\right)^{2}},
$$

where $K_{1}=k_{a+1}+k_{a+2}+\cdots+k_{b-1}$ is the sum of the adjacent momenta between $a$ and $b$ (in the cyclic sense) and $K_{2}=-k_{a}-K_{1}-k_{b}$. (See fig. 1.) In general we label the one-loop integrals by their external legs, following the cyclic ordering around the loop. The parentheses group together those legs of the amplitude which combine together to form a massive leg of the integral. As a more compact notation, we sometimes label the massive legs just by their total momentum (e.g., $K_{1}$ or $K_{2}$ ). See appendix D for more details. We use the same labeling for the coefficients of the integrals.

The explicit form for the integrals (3.10) near $D=4$ [40] is given in eq. (D.4). In terms of these integrals, the $N=4 \mathrm{MHV}$ amplitudes are given by [11]

$$
A_{n ; 1}\left(1^{-}, 2^{-}, 3^{+}, \ldots, n^{+}\right)=\frac{1}{2}\langle 12\rangle^{4} \sum_{\substack{a, b \\ \text { cyclic }}} \alpha_{a K_{1} b K_{2}} \mathcal{I}_{4}^{a K_{1} b K_{2}}+\mathcal{O}(\epsilon)
$$

where the sum is over all integrals with the standard $123 \ldots n$ cyclic ordering of external legs, and 


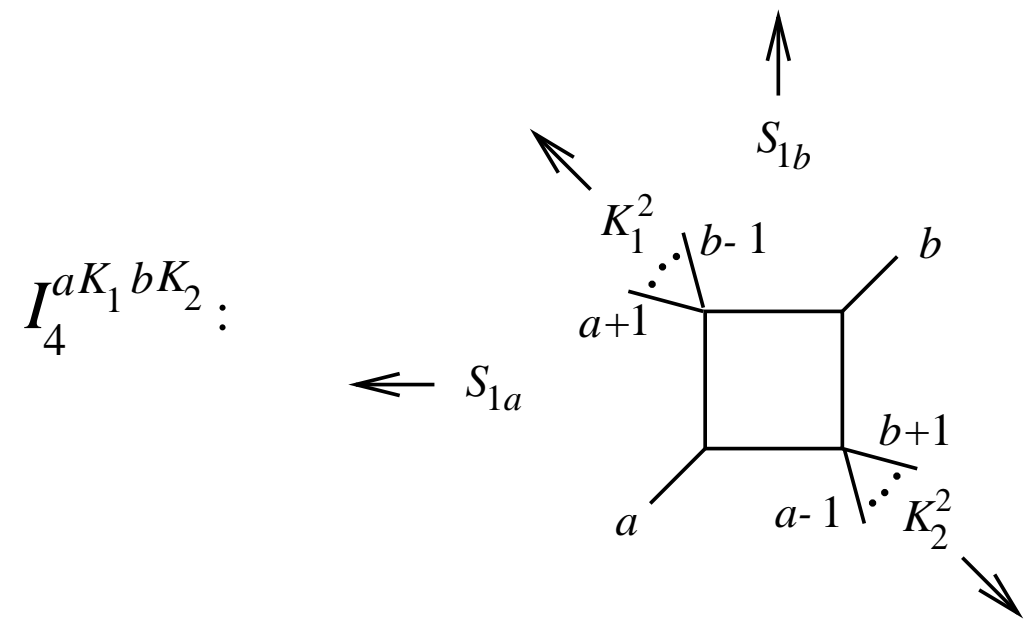

Figure 1: Kinematics of the two-mass box integrals $\mathcal{I}_{4}^{a K_{1} b K_{2}}$ that enter $n$-point MHV amplitudes in both $N=4$ super-Yang-Mills theory and $N=8$ supergravity. Here $a$ and $b$ label the external massless legs for the integral, which coincide with two external momenta for the amplitude, $k_{a}$ and $k_{b}$. The massive legs carry momenta $K_{1}$ and $K_{2}$, which are sums of the remaining external momenta. The four Lorentz invariants are the masses $K_{1}^{2}$ and $K_{2}^{2}$, and the Mandelstam invariants $S_{1 a}=\left(K_{1}+k_{a}\right)^{2}$ and $S_{1 b}=\left(K_{1}+k_{b}\right)^{2}$.

over all distinct non-adjacent pairs of massless legs $a, b$. The coefficients of the box integrals are

$$
\alpha_{a K_{1} b K_{2}}=-\frac{\left(K_{1}+k_{a}\right)^{2}\left(K_{1}+k_{b}\right)^{2}-K_{1}^{2} K_{2}^{2}}{\langle 12\rangle\langle 23\rangle\langle 34\rangle \cdots\langle n 1\rangle}=\frac{\left\langle a^{-}\left|K_{1}\right| b^{-}\right\rangle\left\langle b^{-}\left|K_{2}\right| a^{-}\right\rangle}{\langle 12\rangle\langle 23\rangle\langle 34\rangle \cdots\langle n 1\rangle}=\frac{1}{2} \frac{\operatorname{tr}\left[a K_{1} b K_{2}\right]}{\langle 12\rangle\langle 23\rangle\langle 34\rangle \cdots\langle n 1\rangle},
$$

where $\left\langle i^{-}|K| j^{-}\right\rangle \equiv\left\langle i^{-}|\mathbb{K}| j^{-}\right\rangle$and $\operatorname{tr}\left[a K_{1} b K_{2}\right] \equiv \operatorname{tr}\left[k_{a} \not K_{1} \not k_{b} \not K_{2}\right]$.

For the purpose of facilitating comparisons to the gravity results, an alternative representation for the coefficients is

$$
\alpha_{a K_{1} b K_{2}}=\frac{1}{2} g\left(a, K_{1}, b\right) g\left(b, K_{2}, a\right) \operatorname{tr}\left[a K_{1} b K_{2}\right],
$$

where the functions $g$ are

$$
g\left(a, K_{1}, b\right) \equiv g(a,\{a+1, a+2, \ldots, b-1\}, b)=\frac{1}{\langle a, a+1\rangle\langle a+1, a+2\rangle \cdots\langle b-1, b\rangle} .
$$

For $n=4$, eq. (3.11) is the exact answer, to all orders in $\epsilon$. For $n>4$, the $\mathcal{O}(\epsilon)$ terms contain pentagon and higher-point integrals evaluated in $D=6-2 \epsilon$, with a manifest $\epsilon$ prefactor. These terms are currently known only for $n=5,6[15]$ - see below.

\section{5 'Dimension-Shifting' Relations}

The one-loop MHV amplitudes (3.11) in $N=4$ super-Yang-Mills theory bear a curious 'dimensionshifting' relation to the sequence of one-loop 'all-plus' amplitudes (3.5). The relation between $N=4$ MHV and all-plus amplitudes may be expressed in two different ways [15],

$$
\begin{aligned}
A_{n ; 1}\left(1^{+}, 2^{+}, \ldots, n^{+}\right) & =-2 \epsilon(1-\epsilon)(4 \pi)^{2}\left[\left.\frac{A_{n ; 1}^{N=4}\left(1^{+}, 2^{+}, \ldots, i^{-}, \ldots, j^{-}, \ldots, n^{+}\right)}{\langle i j\rangle^{4}}\right|_{D \rightarrow D+4}\right] \\
& =2 \frac{A_{n ; 1}^{N=4}\left(1^{+}, 2^{+}, \ldots, i^{-}, \ldots, j^{-}, \ldots, n^{+}\right)\left[\mu^{4}\right]}{\langle i j\rangle^{4}}
\end{aligned}
$$


Here ' $D \rightarrow D+4$ ' instructs one to replace the $D$ appearing in the loop-momentum integration measure $d^{D} L$ by $D+4$ (where $D=4-2 \epsilon$ ) in all integrals appearing in $A_{n ; 1}$. The notation ' $\left[\mu^{4}\right]$ ' means that an extra factor of $\mu^{4}$ should be inserted into the numerator of every loop integral in the amplitude. The equivalence of the two forms follows from eq. (3.9). This relation (3.15) has been established for $n=4,5,6$ but remains a conjecture for $n \geq 7$.

A few comments about the dimension-shifting relation (3.15) are in order. First of all, the manifest symmetry of the all-plus amplitudes on the left-hand side of the relation under the cyclic substitution $1 \rightarrow 2 \rightarrow \ldots \rightarrow n \rightarrow 1$ is also present on the right-hand side, as a consequence of the SWI (2.5). Secondly, in four dimensions the all-plus amplitudes have no unitarity cuts, and hence are pure rational functions [12,13], while the $N=4 \mathrm{MHV}$ amplitudes have cuts in all channels. These facts are consistent with eq. (3.15) because of the manifest $\epsilon$ on the right-hand side (in the first form of the relation): Only the ultraviolet $1 / \epsilon$ poles in the higher-dimensional box, pentagon, etc., integrals for the $N=4$ amplitudes contribute as $D \rightarrow 4(\epsilon \rightarrow 0)$, and these have rational-function coefficients.

The dimension-shifting relation (3.15) is a statement about all orders in $\epsilon$, so its complete verification requires all-orders evaluation of both sides. The all- $n$ formulae (3.11) and (3.5), which are only valid through $\mathcal{O}\left(\epsilon^{0}\right)$, are not sufficient for this purpose. However, for $n=4,5,6$ the relation was verified in ref. [15], with the all-plus amplitudes given in eq. (3.7). The $N=4$ amplitudes may be obtained from this equation by applying the dimension-shifting formula; the net effect on the integrals is to remove four powers of $\mu$ from their arguments.

For the all-plus amplitudes, the pentagon terms in eq. (3.7) do contribute as $\epsilon \rightarrow 0$, since these integrals are ultraviolet divergent at $D=10$, canceling the $\epsilon$ prefactor in eq. (3.9). On the other hand, for the $N=4$ amplitudes the pentagon and hexagon terms are finite and do not cancel the overall $\epsilon$ implied by the remaining $\mu^{2}$ arguments. This leaves only box integrals in the expression (3.11) for the $N=4 \mathrm{MHV}$ amplitudes in $D=4-2 \epsilon$ as $\epsilon \rightarrow 0$.

Eqs. (3.7) — or rather the cuts of eqs. (3.7) in various channels — will play a role in section 4 as we construct the analogous amplitudes in $N=8$ supergravity and pure (or self-dual) gravity, using in part the KLT relations between tree amplitudes on either side of the cuts.

In direct analogy to eq. (3.15), one may conjecture a relation between one-loop MHV amplitudes in $N=8$ supergravity, and all-plus amplitudes in pure gravity [15]:

$$
\begin{aligned}
M_{n}\left(1^{+}, 2^{+}, \ldots, n^{+}\right) & =-2 \epsilon(1-\epsilon)(2-\epsilon)(3-\epsilon)(4 \pi)^{4}\left[\left.\frac{M_{n}^{N=8}\left(1^{+}, 2^{+}, \ldots, i^{-}, \ldots, j^{-}, \ldots, n^{+}\right)}{\langle i j\rangle^{8}}\right|_{D \rightarrow D+8}\right] \\
& =2 \frac{M_{n}^{N=8}\left(1^{+}, 2^{+}, \ldots, i^{-}, \ldots, j^{-}, \ldots, n^{+}\right)\left[\mu^{8}\right]}{\langle i j\rangle^{8}} .
\end{aligned}
$$

This equation respects the heuristic relation 'gravity $\sim$ (gauge theory) $)^{2}$, since the $\mu^{4}$ gauge theory factor becomes a $\mu^{8}$ factor in the gravity case. Here $M_{n}^{N=8}$ refers to an entire $N=8$ multiplet circulating in the loop, while $M_{n}\left(1^{+}, 2^{+}, \ldots, n^{+}\right)$gives the contribution of a graviton in the loop. For the same reasons as in the gauge case, this $M_{n}$ could equally well be calculated with a massless scalar in the loop instead [49]. In ref. [15] eq. (3.16) was only verified for the simplest case, $n=4$. 
In the following section we shall see that it holds for $n=5$ and 6 as well, thus strengthening the all- $n$ conjecture.

\section{Cut Construction of One-Loop MHV $N=8$ Supergravity and All-Plus Gravity Amplitudes}

In this section we construct the one-loop $n$-point all-plus gravity amplitudes from their unitarity cuts, for $n \leq 6$. (The case of $n=4$ has been computed previously in refs. [49, 50].) Then we exploit the gauge dimension-shifting relations of section 3.5 to obtain the $N=8 \mathrm{MHV}$ supergravity amplitudes. These calculations will provide a firm basis from which we shall construct ansatze for an arbitrary number of external legs in section 6 , using the soft and collinear behavior of gravity amplitudes to be discussed in section 5 .

\subsection{Brief Review of Cutting Method}

The cutting method that we use has been discussed extensively for the case of gauge theory amplitudes, and reviewed in ref. [16], so we only briefly describe it. This is a proven technology, having been used in the calculation of analytic expressions for the QCD one-loop helicity amplitudes for $Z \rightarrow 4$ partons [51], in the construction of infinite sequences [12,11,34] of one-loop MHV amplitudes and for two-loop four-point amplitudes in maximally supersymmetric cases [52,6]. This technique allows for a complete reconstruction of the amplitudes from the cuts, provided that all cuts are known in arbitrary dimension. Because on-shell expressions are used throughout, gauge invariance, Lorentz covariance and unitarity are manifest.

The unitarity cuts of one-loop amplitudes are given simply by phase-space integrals of products of tree amplitudes, summing over all intermediate states that can cross the cut. For example, the cut in the channel carrying momentum $k_{m_{1}}+\cdots+k_{m_{2}}$ for $M_{n}(1,2, \ldots, n)$ is given by

$$
\begin{aligned}
C_{m_{1} \ldots m_{2}}=i \sum_{\lambda_{1}, \lambda_{2}} \int d \operatorname{LIPS}\left(-L_{1}, L_{2}\right) & M_{m_{2}-m_{1}+3}^{\mathrm{tree}}\left(\left(-L_{1}\right)^{-\lambda_{1}}, m_{1}, \ldots, m_{2}, L_{2}^{\lambda_{2}}\right) \\
\times & M_{n+m_{1}-m_{2}+1}^{\mathrm{tree}}\left(\left(-L_{2}\right)^{-\lambda_{2}}, m_{2}+1, \ldots, m_{1}-1, L_{1}^{\lambda_{1}}\right),
\end{aligned}
$$

where the integration is over the two-particle $D$-dimensional Lorentz-invariant phase-space, and $\lambda_{1,2}$ denote the helicity/particle-type of the states crossing the cut. (Polarization labels for the external graviton states have been suppressed.) One can replace [16] the phase-space integral with an unrestricted loop momentum integral $\int d^{D} L$, yet continue to apply the on-shell conditions $L_{1}^{2}=$ $L_{2}^{2}=0$, so long as one remembers that only functions with a cut in the given channel are reliably computed in this way. (The positive energy conditions are automatically imposed with the use of Feynman propagators.)

A principal advantage of the cutting approach for gauge theory calculations is that the tree amplitudes on either side of the cut can be simplified before attempting to evaluate the cut integral [16]. In the case of gravity, the KLT relations provide convenient representations of the tree

amplitudes. In the supersymmetric case, on-shell supersymmetry Ward identities can also be used to 
reduce the amount of work required. (To maintain the supersymmetry cancellations the dimensional regularization scheme should not alter the number of states from their four-dimensional values [53].)

For the non-supersymmetric all-plus calculation, the SWI (2.4) allow us to replace gravitons or any other massless particles in the loop with massless scalars [49]. That is, at one loop we have,

$$
M_{n}^{\text {any states }}\left(1^{+}, 2^{+}, \ldots, n^{+}\right)=N_{s} M_{n}^{\text {scalar }}\left(1^{+}, 2^{+}, \ldots, n^{+}\right),
$$

where $N_{s}$ is the number of bosonic states minus fermionic states circulating in the loop in $M_{n}^{\text {any states }}$. (We have taken the normalization of the 'scalar' amplitude to be that for a single real scalar state.) For scalars (or fermions) crossing the cuts, a detailed study of the effect of the $D$-dimensional loop momentum has previously been presented $[36,16,15]$, and the requisite gauge theory tree amplitudes, where the scalar carries non-zero momenta in the extra $(-2 \epsilon)$ dimensions, have been computed for $n \leq 6$ [15]. We shall obtain the scalar+graviton tree amplitudes from the scalar+gluon tree amplitudes using the KLT relations. Thus we shall be able to directly evaluate the cuts for the all-plus amplitudes to all orders in $\epsilon$, which in turn gives the full amplitudes to all orders in $\epsilon[54,16,36]$.

One good way to obtain the $N=8 \mathrm{MHV}$ amplitudes with up to six legs, through $\mathcal{O}\left(\epsilon^{0}\right)$, is to make use of a 'cut constructible' criterion that allows one to use four-dimensional momenta in the cuts without introducing any errors in $\mathcal{O}\left(\epsilon^{0}\right)$ rational functions, assuming that certain power counting criteria are satisfied [34]. In the present case, however, we can avoid explicit computations of the $N=8 \mathrm{MHV}$ cuts by instead obtaining the $N=8$ amplitudes from the all-plus amplitudes using the gravitational version (3.16) of the gauge theory 'dimension-shifting' results presented in section 3.5. We now explain how the gravitational relation can be derived from the gauge theory one.

\subsection{Dimension-Shifting Relations between Gravity Cuts}

In ref. [15] the dimension-shifting formula (3.16) was shown to hold for the four-graviton amplitudes, and was conjectured to hold for $n$-point amplitudes. Here we demonstrate that it does hold at $n$ points, if the gauge theory relation (3.15) holds at $n$-points. Since the latter relation has been proven for $n \leq 6$, this establishes the gravity dimension-shifting relation (3.16) up to six points, but leaves the $n \geq 7$ cases as a conjecture.

We begin with the cuts of the gauge theory dimension-shifting formula (3.15),

$$
\begin{aligned}
& A_{m+2}^{\text {tree }}\left(-L_{1}^{s}, i_{1}^{+}, i_{2}^{+}, \ldots, L_{2}^{s}, \ldots, i_{m}^{+}\right) \times A_{n-m+2}^{\text {tree }}\left(-L_{2}^{s}, i_{m+1}^{+}, i_{m+2}^{+}, \ldots, L_{1}^{s}, \ldots, i_{n}^{+}\right) \\
& =\frac{\mu^{4}}{\langle i j\rangle^{4}} \sum_{N=4 \text { states }} A_{m+2}^{\text {tree }}\left(-L_{1}, i_{1}^{+}, i_{2}^{+}, \ldots, L_{2}, \ldots, i_{m}^{+}\right) \times A_{n-m+2}^{\text {tree }}\left(-L_{2}, i_{m+1}^{+}, i_{m+2}^{+}, \ldots, L_{1}, \ldots, i_{n}^{+}\right),
\end{aligned}
$$

where the superscript $s$ denotes a scalar line, the sum on the right-hand side runs over all $N=4$ super-Yang-Mills states that can cross the cut, and we have suppressed the $\lambda_{1,2}$ helicity/state labels for these states. (The reason the ' 2 ' in eq. (3.15) has disappeared from eq. (4.3) is that $A_{n ; 1}$ in the former equation corresponds to 2 real scalars circulating in the loop.) Eq. (3.15) was originally derived in its loop-momentum integrated version. Nevertheless, it turns out that the manipulations 
used in ref. [15] to verify the relations between the amplitudes can be arranged so as not to introduce any total derivatives. This means that eq. (3.15) holds point-by-point in the integrands.

Also, the cuts of eq. (3.15) (a leading-in- $N_{c}$ equation) only correspond directly to the configurations where the cut loop momenta $L_{1}$ and $L_{2}$ are adjacent, i.e. $A_{m+2}^{\text {tree }}\left(-L_{1}^{s}, i_{1}^{+}, \ldots, i_{m}^{+}, L_{2}^{s}\right) \times$ $A_{n-m+2}^{\text {tree }}\left(-L_{2}^{s}, i_{m+1}^{+}, \ldots, i_{n}^{+}, L_{1}^{s}\right)$ on the left-hand side of eq. (4.3). However, it is possible to obtain all the other permutations of this equation, by using the following relation among color-ordered tree amplitudes [55],

$$
A^{\text {tree }}(1,\{\alpha\}, 2,\{\beta\})=(-1)^{n_{\beta}} \sum_{\sigma \in O P\{\alpha\}\left\{\beta^{T}\right\}} A^{\text {tree }}\left(1, \sigma\left(\{\alpha\}\left\{\beta^{T}\right\}\right), 2\right),
$$

where $n_{\beta}$ is the number of elements in $\{\beta\}$, the set $\beta^{T}$ is $\beta$ with the ordering reversed, and $O P\{\alpha\}\left\{\beta^{T}\right\}$ is the set of all permutations of $\{\alpha\} \cup\left\{\beta^{T}\right\}$ that preserve the ordering of elements within each of the two sets. Eq. (4.4) can be inserted twice each into the left- and right-hand sides of eq. (4.3), in order to reduce the general case to the case where $L_{1}$ and $L_{2}$ are adjacent.

We may now use the $n$-point KLT equation (A.1) to rewrite the cuts of the all-plus gravity amplitudes in terms of gauge theory cuts,

$$
\begin{aligned}
& M_{m+2}^{\text {tree }}\left(-L_{1}^{s}, 1^{+}, 2^{+}, \ldots, m^{+}, L_{2}^{s}\right) \times M_{n-m+2}^{\text {tree }}\left(-L_{2}^{s},(m+1)^{+}, \ldots, n^{+}, L_{1}^{s}\right) \\
& =\left(\sum_{\text {perms }} f \bar{f} A_{m+2}^{\text {tree }}\left(-L_{1}^{s}, 1^{+}, 2^{+}, \ldots, m^{+}, L_{2}^{s}\right) A_{m+2}^{\text {tree }}\left(i_{1}^{+}, \ldots,-L_{1}^{s}, m^{+}, i_{m-1}^{+}, \ldots, L_{2}^{s}\right)\right) \\
& \quad \times\left(\sum_{\text {perms }} f^{\prime} \bar{f}^{\prime} A_{n-m+2}^{\text {tree }}\left(-L_{2}^{s},(m+1)^{+}, \ldots, n^{+}, L_{1}^{s}\right) A_{n-m+2}^{\text {tree }}\left(i_{m+1}^{+}, \ldots,-L_{2}^{s}, n^{+}, i_{n-1}^{+}, \ldots, L_{1}^{s}\right)\right),
\end{aligned}
$$

where 'perms' and 'perms' stand for the full sum over KLT permutations in eq. (A.1), and $f, \bar{f}, f^{\prime}$ and $\bar{f}^{\prime}$ are the functions $f, \bar{f}$ defined in eq. (A.2), for the appropriate sets of arguments. (The KLT equations hold in any dimension $D \leq 10$ where string constructions exist and may be analytically continued to arbitrary dimensions.) After rearranging the right-hand side of eq. (4.5) and applying the cut version of the gauge theory dimension-shifting formula (4.3), we obtain

$$
\begin{aligned}
& M_{m+2}^{\text {tree }}\left(-L_{1}^{s}, 1^{+}, 2^{+}, \ldots, m^{+}, L_{2}^{s}\right) \times M_{n-m+2}^{\text {tree }}\left(-L_{2}^{s},(m+1)^{+}, \ldots, n^{+}, L_{1}^{s}\right) \\
& =\sum_{\text {perms }} \sum_{\text {perms }} f \bar{f} f^{\prime} \bar{f}^{\prime}\left[A_{m+2}^{\text {tree }}\left(-L_{1}^{s}, 1^{+}, 2^{+}, \ldots, m^{+}, L_{2}^{s}\right) A_{n-m+2}^{\text {tree }}\left(-L_{2}^{s},(m+1)^{+}, \ldots, n^{+}, L_{1}^{s}\right)\right] \\
& \times\left[A_{m+2}^{\mathrm{tree}}\left(i_{1}^{+}, \ldots,-L_{1}^{s}, m^{+}, i_{m-1}^{+}, \ldots, L_{2}^{s}\right) A_{n-m+2}^{\mathrm{tree}}\left(i_{m+1}^{+}, \ldots,-L_{2}^{s}, n^{+}, i_{n-1}^{+}, \ldots, L_{1}^{s}\right)\right] \\
& =\sum_{\text {perms }} \sum_{\text {perms }} f \bar{f} f^{\prime} \bar{f}^{\prime} \\
& \times \sum_{N=4 \text { states }}\left[\frac{\mu^{4}}{\langle i j\rangle^{4}} A_{m+2}^{\text {tree }}\left(-L_{1}, 1^{+}, 2^{+}, \ldots, m^{+}, L_{2}\right) A_{n-m+2}^{\text {tree }}\left(-L_{2},(m+1)^{+}, \ldots, n^{+}, L_{1}\right)\right] \\
& \times \sum_{N=4 \text { states }}\left[\frac{\mu^{4}}{\langle i j\rangle^{4}} A_{m+2}^{\text {tree }}\left(i_{1}^{+}, \ldots,-L_{1}, m^{+}, i_{m-1}^{+}, \ldots, L_{2}\right) A_{n-m+2}^{\text {tree }}\left(i_{m+1}^{+}, \ldots,-L_{2}, n^{+}, i_{n-1}^{+}, \ldots, L_{1}\right)\right] .
\end{aligned}
$$

As discussed in section 2.1, in terms of a $D=4$ decomposition of states the double sum over the 16 $N=4$ super-Yang-Mills states may be reassembled as a single sum over the $256 N=8$ supergravity 
states. (In higher dimensions up to $D=10$, the sum over $N=4$ states can be reassembled into a sum over the appropriate multiplet in the higher dimensional theory.) This yields,

$$
\begin{aligned}
& M_{m+2}^{\text {tree }}\left(-L_{1}^{s}, 1^{+}, \ldots, m^{+}, L_{2}^{s}\right) \times M_{n-m+2}^{\text {tree }}\left(-L_{2}^{s},(m+1)^{+}, \ldots, n^{+}, L_{1}^{s}\right) \\
& \quad=\frac{\mu^{8}}{\langle i j\rangle^{8}} \sum_{N=8 \text { states }} M_{m+2}^{\text {tree }}\left(-L_{1}, 1^{+}, 2^{+}, \ldots, m^{+}, L_{2}\right) \times M_{n-m+2}^{\text {tree }}\left(-L_{2},(m+1)^{+}, \ldots, n^{+}, L_{1}\right) .
\end{aligned}
$$

Note that the precise details of which permutation sums are included, or what exactly the $f$ functions are, are unimportant in the derivation of eq. (4.7), because the same permutations and $f$ functions appear in the KLT expressions for any of the tree amplitudes appearing in the cuts, regardless of the particle type of the states crossing the cut. Since eq. (4.7) can be used to obtain all cuts of the amplitudes and is valid to all orders in $\epsilon$, the gravity dimension-shifting relation (3.16) is established for all values of $n$ for which eq. (4.3) has been proven (currently this is for $n \leq 6$ ).

The relationships between the infinite sequences of one-loop all-plus (or self-dual) amplitudes and MHV amplitudes in maximally supersymmetric theories, and between gravity and gauge theory, are summarized in fig. 2. The horizontal arrows correspond to the gauge-gravity relations that follow from the KLT equations, and the vertical arrows represent the dimension-shifting relations.

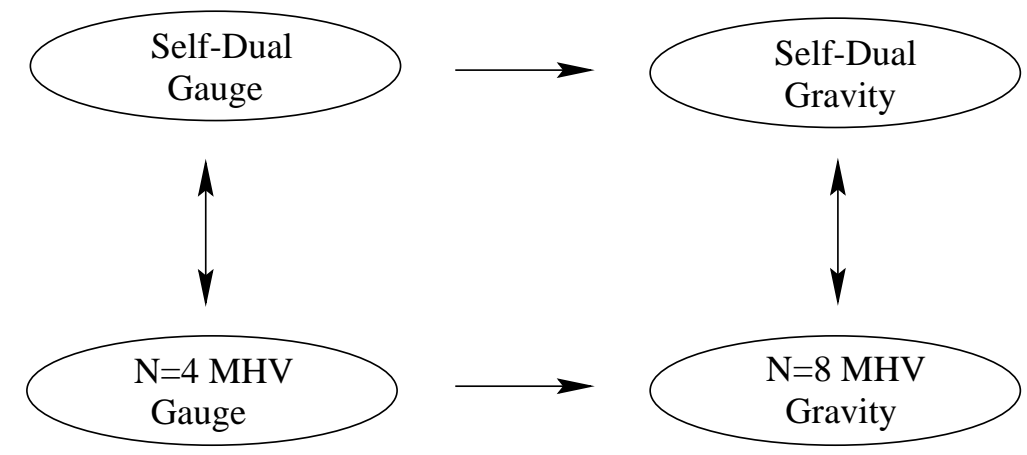

Figure 2: Relations between infinite sequences of one-loop amplitudes in four different theories. The vertical arrows correspond to the 'dimension-shifting' relations of ref. [15], within (super-) Yang-Mills theory and (super-) gravity. (These remain a conjecture for $n \geq 7$ legs.) The horizontal arrows correspond to the gauge-gravity relations which follow from the KLT equations.

Using eq. (3.16) it is then sufficient to calculate the all-plus graviton amplitudes as a function of $\epsilon$ (i.e., for arbitrary $D=4-2 \epsilon$ ), in order to obtain the $N=8$ supergravity MHV amplitudes.

\subsection{All-Plus Amplitudes for $n \leq 6$}

As a warmup we first calculate the four-graviton all-plus amplitude, before proceeding to the fiveand six-graviton cases. Using eq. (4.2) we can replace the graviton in the loop with two real scalars. Thus, the cut in the $s_{12}$ channel is

$$
\left.M_{4}\left(1^{+}, 2^{+}, 3^{+}, 4^{+}\right)\right|_{s_{12}-\text { cut }}=\left.\int \frac{d^{D} L_{1}}{(2 \pi)^{D}} \frac{i}{L_{1}^{2}} M_{4}^{\text {tree }}\left(-L_{1}^{s}, 1^{+}, 2^{+}, L_{3}^{s}\right) \frac{i}{L_{3}^{2}} M_{4}^{\text {tree }}\left(-L_{3}^{s}, 3^{+}, 4^{+}, L_{1}^{s}\right)\right|_{s_{12}-\text { cut }},
$$

where the superscript $s$ denotes that the cut lines are scalars, and $L_{3}=L_{1}-k_{1}-k_{2}$. An overall 2 , from two real scalars propagating in the loop, is canceled by an identical-particle phase-space factor 
of $1 / 2$. Using the KLT expressions (2.2) we may replace the gravity tree amplitudes appearing in the cuts with products of gauge theory amplitudes. The required gauge theory tree amplitudes, with two external scalar legs and two gluons, are relatively simple to obtain $[36,16]$,

$$
\begin{aligned}
& A_{4}^{\text {tree }}\left(-L_{1}^{s}, 1^{+}, 2^{+}, L_{3}^{s}\right)=i \frac{\mu^{2}[12]}{\langle 12\rangle\left[\left(\ell_{1}-k_{1}\right)^{2}-\mu^{2}\right]}, \\
& A_{4}^{\text {tree }}\left(-L_{1}^{s}, 1^{+}, L_{3}^{s}, 2^{+}\right)=-i \frac{\mu^{2}[12]}{\langle 12\rangle}\left[\frac{1}{\left(\ell_{1}-k_{1}\right)^{2}-\mu^{2}}+\frac{1}{\left(\ell_{1}-k_{2}\right)^{2}-\mu^{2}}\right],
\end{aligned}
$$

where the gluon momenta are four-dimensional, but the scalar momenta are allowed to have a $(-2 \epsilon)$ dimensional component $\vec{\mu}$, with $\vec{\mu} \cdot \vec{\mu}=\mu^{2}>0$. The overall factor of $\mu^{2}$ appearing in these tree amplitudes means that they vanish in the four-dimensional limit, in accord with the SWI (2.4). In the KLT relation (2.2), one of the propagators cancels, leaving

$$
M_{4}\left(-L_{1}^{s}, 1^{+}, 2^{+}, L_{3}^{s}\right)=-i\left(\frac{\mu^{2}[12]}{\langle 12\rangle}\right)^{2}\left[\frac{1}{\left(\ell_{1}-k_{1}\right)^{2}-\mu^{2}}+\frac{1}{\left(\ell_{1}-k_{2}\right)^{2}-\mu^{2}}\right] .
$$

Inserting eq. (4.10) and the permuted formula for $M_{4}\left(-L_{3}^{s}, 3^{+}, 4^{+}, L_{1}^{s}\right)$ into the cut (4.8) yields

$$
\begin{aligned}
&\left.M_{4}\left(1^{+}, 2^{+}, 3^{+}, 4^{+}\right)\right|_{s_{12}-\text { cut }} \\
&=\frac{[12]^{2}[34]^{2}}{\langle 12\rangle^{2}\langle 34\rangle^{2}} \int \frac{d^{4} \ell}{(2 \pi)^{4}} \int \frac{d^{-2 \epsilon} \mu}{(2 \pi)^{-2 \epsilon}} \mu^{8} \frac{1}{\ell^{2}-\mu^{2}}\left[\frac{1}{\left(\ell-k_{1}\right)^{2}-\mu^{2}}+\frac{1}{\left(\ell-k_{2}\right)^{2}-\mu^{2}}\right] \\
& \times\left.\frac{1}{\left(\ell-k_{1}-k_{2}\right)^{2}-\mu^{2}}\left[\frac{1}{\left(\ell+k_{4}\right)^{2}-\mu^{2}}+\frac{1}{\left(\ell+k_{3}\right)^{2}-\mu^{2}}\right]\right|_{s_{12}-\text { cut }},
\end{aligned}
$$

which corresponds to a sum of $(12-2 \epsilon)$-dimensional scalar integrals, using (3.9). By symmetry, the other cuts are the same up to relabelings. Combining all three cuts into a single function that has the correct cuts in all channels yields

$$
M_{4}\left(1^{+}, 2^{+}, 3^{+}, 4^{+}\right)=2 \frac{[12]^{2}[34]^{2}}{\langle 12\rangle^{2}\langle 34\rangle^{2}}\left(\mathcal{I}_{4}^{1234}\left[\mu^{8}\right]+\mathcal{I}_{4}^{3124}\left[\mu^{8}\right]+\mathcal{I}_{4}^{2314}\left[\mu^{8}\right]\right),
$$

where

$$
\mathcal{I}_{4}^{1234}\left[\mu^{8}\right]=\int \frac{d^{4} \ell}{(2 \pi)^{4}} \int \frac{d^{-2 \epsilon} \mu}{(2 \pi)^{-2 \epsilon}} \mu^{8} \frac{1}{\left[\ell^{2}-\mu^{2}\right]\left[\left(\ell-k_{1}\right)^{2}-\mu^{2}\right]\left[\left(\ell-k_{1}-k_{2}\right)^{2}-\mu^{2}\right]\left[\left(\ell+k_{4}\right)^{2}-\mu^{2}\right]}
$$

is the scalar box integral with the external legs arranged in the order 1234. The two other scalar integrals that appear correspond to the two other distinct orderings of the four external legs. (See appendix D for our notation for general one-loop integrals.) Using eq. (4.2), this result can be applied to any set of massless fields circulating in the loop.

The spinor factor $[12]^{2}[34]^{2} /\left(\langle 12\rangle^{2}\langle 34\rangle^{2}\right)$ in eq. (4.12) is actually completely symmetric, although not manifestly so. By rewriting this factor and using eq. (D.9) for the box integral, the final one-loop result in $D=4$ is

$$
M_{4}\left(1^{+}, 2^{+}, 3^{+}, 4^{+}\right)=-\frac{i}{(4 \pi)^{2}}\left(\frac{s t}{\langle 12\rangle\langle 23\rangle\langle 34\rangle\langle 41\rangle}\right)^{2} \frac{s^{2}+t^{2}+u^{2}}{120}+\mathcal{O}(\epsilon),
$$


in agreement with a previous calculation [50].

For the purpose of constructing an ansatz for $n \geq 7$, it is useful to write the $n=4$ amplitude as

$$
\begin{aligned}
M_{4}\left(1^{+}, 2^{+}, 3^{+}, 4^{+}\right)= & -\frac{i}{(4 \pi)^{2}} \frac{1}{480}\left[h(1,\{3\}, 2) h(2,\{4\}, 1) \operatorname{tr}^{3}[1324]\right. \\
& \left.+h(1,\{2\}, 3) h(3,\{4\}, 1) \operatorname{tr}^{3}[1234]+h(1,\{2\}, 4) h(4,\{3\}, 1) \operatorname{tr}^{3}[1243]\right]+\mathcal{O}(\epsilon),
\end{aligned}
$$

where $\operatorname{tr}\left[i_{1} i_{2} i_{3} i_{4}\right] \equiv \operatorname{tr}\left[\not k_{i_{1}} \not k_{i_{2}} \not k_{i_{3}} \not k_{i_{4}}\right]$ and

$$
h(a,\{1\}, b)=\frac{1}{\langle a 1\rangle^{2}\langle 1 b\rangle^{2}} .
$$

Next we compute $M_{5}\left(1^{+}, 2^{+}, 3^{+}, 4^{+}, 5^{+}\right)$to all orders in $\epsilon$. Its total symmetry implies that the $s_{12}$ cut again suffices for its complete reconstruction. Thus we require the tree amplitudes for two scalars and either two or three gravitons, $M_{4}^{\text {tree }}\left(-L_{1}^{s}, 1^{+}, 2^{+}, L_{3}^{s}\right)$ from eq. (4.10), and $M_{5}^{\text {tree }}\left(-L_{3}^{s}, 3^{+}, 4^{+}, 5^{+}, L_{1}^{s}\right)$, which may be constructed from the gauge amplitudes for two scalars and three gluons [15],

$$
\begin{gathered}
A_{5}^{\text {tree }}\left(L_{1}^{s},-L_{3}^{s}, 3^{+}, 4^{+}, 5^{+}\right)=i \mu^{2} \frac{\left\langle 5^{+}\left|(3+4) \ell_{3}\right| 3^{-}\right\rangle}{\left(L_{3}-k_{3}\right)^{2}\langle 34\rangle\langle 45\rangle\left(L_{1}+k_{5}\right)^{2}}, \\
A_{5}^{\text {tree }}\left(L_{1}^{s}, 3^{+},-L_{3}^{s}, 4^{+}, 5^{+}\right)=-A_{5}^{\text {tree }}\left(L_{1}^{s},-L_{3}^{s}, 3^{+}, 4^{+}, 5^{+}\right)-A_{5}^{\text {tree }}\left(L_{1}^{s},-L_{3}^{s}, 4^{+}, 3^{+}, 5^{+}\right) \\
-A_{5}^{\text {tree }}\left(L_{1}^{s},-L_{3}^{s}, 4^{+}, 5^{+}, 3^{+}\right),
\end{gathered}
$$

using the five-point KLT relation (2.2). The second equation (a special case of eq. (4.4)) follows from the $U(1)$ decoupling identity $[28,37,31]$.

After applying several spinor-product identities to the right-hand side of the five-point KLT relation, we obtain the manifestly symmetric form

$$
\begin{gathered}
M_{5}^{\text {tree }}\left(-L_{3}^{s}, 3^{+}, 4^{+}, 5^{+}, L_{1}^{s}\right)=-i \frac{\mu^{4}}{\langle 34\rangle^{2}\langle 35\rangle^{2}\langle 45\rangle^{2}}\left[\frac{s_{34} s_{45}}{\left(L_{3}-k_{3}\right)^{2}\left(L_{1}+k_{5}\right)^{2}}\left(\left\langle 5^{-}\left|3(4+5) \ell_{1}\right| 5^{-}\right\rangle+\mu^{2} s_{35}\right)\right. \\
+\mathcal{P}(3,4,5)] .
\end{gathered}
$$

Inserting eqs. (4.10) and (4.18) into the $s_{12}$-channel cut of the five-point all-plus amplitude gives

$$
\begin{aligned}
\left.M_{5}\left(1^{+}, 2^{+}, \ldots, 5^{+}\right)\right|_{s_{12} \text {-cut }}=\frac{[12]^{2}}{\langle 12\rangle^{2}}\left\langle\frac{d^{D} L}{\langle 34\rangle^{2}\langle 35\rangle^{2}\langle 45\rangle^{2}} \mu^{8}\right. \\
\times\left\{\frac { 1 } { ( 2 \pi ) ^ { D } } \left[\frac{s_{34} s_{45}}{L_{4}^{2} L_{5}^{2}}\left(\left\langle 5^{-}\left|3(4+5) \ell_{1}\right| 5^{-}\right\rangle+\mu^{2} s_{35}\right)\right.\right. \\
+\mathcal{P}(3,4,5)]+\mathcal{P}(1,2)\}\left.\right|_{s_{12} \text {-cut }},
\end{aligned}
$$

where $L_{i}=L-k_{1}-\cdots-k_{i-1}$ and $L_{i}^{2}=\ell_{i}^{2}-\mu^{2}$. The $L_{1}^{2} L_{2}^{2} L_{3}^{2} L_{4}^{2} L_{5}^{2}$ denominator factors signal the presence of a pentagon integral with a 12345 cyclic ordering of external legs. All other orderings appearing in the $s_{12}$ cut are generated by the permutation sums. 
By using standard integration formulas, and combining all cuts into a single function, we find that the five-point all-plus gravity amplitude may be put into a form similar to the all-plus gauge amplitude (eq. (3.5)),

$$
M_{5}\left(1^{+}, 2^{+}, \ldots, 5^{+}\right)=\beta_{123(45)} \mathcal{I}_{4}^{123(45)}\left[\mu^{8}\right]-2 \frac{[12][23][34][45][51]}{\langle 12\rangle\langle 23\rangle\langle 34\rangle\langle 45\rangle\langle 51\rangle} \mathcal{I}_{5}^{12345}\left[\mu^{10}\right]+\text { perms }
$$

where the permutation sum is over all distinct one-mass box integrals and massless pentagon integrals (no cyclic ordering is imposed, in contrast to the gauge case). There are 30 different box-integral terms and 12 pentagons in the sum. The box coefficient is

$$
\beta_{123(45)}=-\frac{[12]^{2}[23]^{2}[45]}{\langle 14\rangle\langle 15\rangle\langle 34\rangle\langle 35\rangle\langle 45\rangle} .
$$

We can rewrite this coefficient in terms of the one given for the five-point all-plus gauge amplitude in eq. (3.7) (or equivalently, for the MHV amplitude in $N=4$ super-Yang-Mills theory in eq. (3.12)):

$$
\begin{aligned}
\beta_{123(45)} & =-s_{45} \frac{s_{12} s_{23}}{\langle 12\rangle\langle 23\rangle\langle 34\rangle\langle 45\rangle\langle 51\rangle} \frac{s_{12} s_{23}}{\langle 12\rangle\langle 23\rangle\langle 35\rangle\langle 54\rangle\langle 41\rangle} \\
& =-s_{45} \alpha_{123(45)} \alpha_{123(54)} .
\end{aligned}
$$

This relation is reminiscent of the tree-level four-point KLT relation in eq. (2.2), in that

(1) a quantity in gravity is expressed as a product of gauge quantities,

(2) one $s_{i j}$ appears as a prefactor on the gauge side of the relation, and

(3) the indices on the $s_{i j}$ coincide with the arguments which are permuted between the two gaugetheory factors.

Through $\mathcal{O}\left(\epsilon^{0}\right)$ the expression for the amplitude can be simplified considerably by non-trivial rearrangements to yield,

$$
\begin{aligned}
M_{5}\left(1^{+}, 2^{+}, 3^{+}, 4^{+}, 5^{+}\right) & \\
& =\frac{i}{(4 \pi)^{2}} \frac{1}{960} h(1,\{2\}, 3) h(3,\{4,5\}, 1) \operatorname{tr}^{3}[123(4+5)]+\text { perms }+\mathcal{O}(\epsilon),
\end{aligned}
$$

where $\operatorname{tr}[\cdots(i+j) \cdots] \equiv \operatorname{tr}\left[\cdots\left(\not k_{i}+\not k_{j}\right) \cdots\right], h(a,\{1\}, b)$ is defined in eq. (4.16),

$$
h(a,\{1,2\}, b)=\frac{[12]}{\langle 12\rangle\langle a 1\rangle\langle 1 b\rangle\langle a 2\rangle\langle 2 b\rangle},
$$

and the sum is over $10 \times 3=30$ distinct permutations. (There are $\left(\begin{array}{l}5 \\ 2\end{array}\right)=10$ possible choices for the pair of arguments in braces in the second $h$ function, and for each of these there are 3 more choices for the argument in braces in the first $h$ function.)

We have also obtained the six-point all-plus amplitude to all orders in $\epsilon$ from the cuts. The result of this computation is

$$
M_{6}\left(1^{+}, \ldots, 6^{+}\right)=\beta_{1(23) 4(56)} \mathcal{I}_{4}^{1(23) 4(56)}\left[\mu^{8}\right]+\beta_{123(456)} \mathcal{I}_{4}^{123(456)}\left[\mu^{8}\right]+\rho_{1234(56)} \mathcal{I}_{5}^{1234(56)}\left[\mu^{10}\right]+\text { perms }
$$


where the sum over permutations again runs over all distinct integral functions. The coefficients of the integrals are

$$
\begin{aligned}
\beta_{1(23) 4(56)=} & \frac{[23]}{\langle 23\rangle} \frac{[56]}{\langle 56\rangle} \frac{\left\langle 1^{-}|(2+3)| 4^{-}\right\rangle^{2}\left\langle 4^{-}|(5+6)| 1^{-}\right\rangle^{2}}{\langle 12\rangle\langle 24\rangle\langle 13\rangle\langle 34\rangle\langle 45\rangle\langle 51\rangle\langle 46\rangle\langle 61\rangle}, \\
\beta_{123(456)=} & \frac{[12]^{2}[23]^{2}}{\langle 14\rangle\langle 43\rangle\langle 15\rangle\langle 53\rangle\langle 16\rangle\langle 63\rangle} \\
& \quad \times\left(\langle 14\rangle\langle 43\rangle \frac{[45][46]}{\langle 45\rangle\langle 46\rangle}+\langle 15\rangle\langle 53\rangle \frac{[45][56]}{\langle 45\rangle\langle 56\rangle}+\langle 16\rangle\langle 63\rangle \frac{[46][56]}{\langle 46\rangle\langle 56\rangle}\right), \\
\rho_{1234(56)}= & 2 \frac{s_{12} s_{23} s_{34}\left\langle 1^{-}|(2+3)| 4^{-}\right\rangle\left\langle 4^{-}|(5+6)| 1^{-}\right\rangle}{\operatorname{tr}_{5}[1234]}\left[c_{1}+c_{2}+\left.c_{2}\right|_{5 \leftrightarrow 6}\right],
\end{aligned}
$$

where $\operatorname{tr}_{5}[\cdots] \equiv \operatorname{tr}\left[\gamma_{5} \cdots\right]$ and

$$
\begin{aligned}
c_{1} & =\frac{\operatorname{tr}_{+}[1234][56]}{\langle 12\rangle^{2}\langle 23\rangle^{2}\langle 34\rangle^{2}\langle 45\rangle\langle 51\rangle\langle 46\rangle\langle 61\rangle\langle 56\rangle}, \\
c_{2} & =\frac{1}{\operatorname{tr}_{5}[123456]} \frac{[12][23][34][45][56][61]}{\langle 12\rangle\langle 23\rangle\langle 34\rangle\langle 45\rangle\langle 56\rangle\langle 61\rangle} .
\end{aligned}
$$

Again the expression for the amplitude through $\mathcal{O}\left(\epsilon^{0}\right)$ can be simplified, to

$$
\begin{gathered}
M_{6}\left(1^{+}, 2^{+}, 3^{+}, 4^{+}, 5^{+}, 6^{+}\right)=-\frac{i}{(4 \pi)^{2}} \frac{1}{960}\left\{h(1,\{2,3\}, 4) h(4,\{5,6\}, 1) \operatorname{tr}^{3}[1(2+3) 4(5+6)]\right. \\
\left.+h(1,\{2\}, 3) h(3,\{4,5,6\}, 1) \operatorname{tr}^{3}[123(4+5+6)]+\text { perms }\right\}
\end{gathered}
$$

where the permutation sum is over distinct terms and the new $h$ function that appears is

$$
\begin{aligned}
h(a,\{1,2,3\}, b)= & \frac{[12][23]}{\langle 12\rangle\langle 23\rangle\langle a 1\rangle\langle 1 b\rangle\langle a 3\rangle\langle 3 b\rangle}+\frac{[23][31]}{\langle 23\rangle\langle 31\rangle\langle a 2\rangle\langle 2 b\rangle\langle a 1\rangle\langle 1 b\rangle} \\
& +\frac{[31][12]}{\langle 31\rangle\langle 12\rangle\langle a 3\rangle\langle 3 b\rangle\langle a 2\rangle\langle 2 b\rangle} .
\end{aligned}
$$

\section{4 $N=8 \mathrm{MHV}$ Amplitudes from Dimension-Shifting}

By using the gravitational dimension-shifting relation (3.16), we may obtain the $N=8$ four-, five-, and six-point MHV amplitudes from the all-plus (self-dual) amplitudes (4.12), (4.20), and (4.25), by dividing out a factor of $\mu^{8}$ from each integrand and multiplying by an overall factor of $\langle i j\rangle^{8} / 2$, where $i$ and $j$ are the two negative helicity legs. After removing a factor of $\mu^{8}$, the pentagon integrals are no longer ultraviolet divergent and are suppressed by an overall power of $\epsilon$ near $D=4$, since a power of $\mu^{2}$ remains. Hence, the $N=8$ amplitudes through $\mathcal{O}\left(\epsilon^{0}\right)$ are given just by the box integral contributions.

A representation of the four-, five- and six-point amplitudes which is convenient for extending 
the result to an arbitrary number of external legs is

$$
\begin{gathered}
M_{4}^{N=8}\left(1^{-}, 2^{-}, 3^{+}, 4^{+}\right)=\frac{1}{4}\langle 12\rangle^{8}\left[h(1,\{2\}, 3) h(3,\{4\}, 1) \operatorname{tr}^{2}[1234] \mathcal{I}_{4}^{1234}+\text { perms }\right]+\mathcal{O}(\epsilon), \\
\begin{aligned}
M_{5}^{N=8}\left(1^{-}, 2^{-}, 3^{+}, 4^{+}, 5^{+}\right)=-\frac{1}{8}\langle 12\rangle^{8}\left[h(1,\{2\}, 3) h(3,\{4,5\}, 1) \operatorname{tr}^{2}[123(4+5)] \mathcal{I}_{4}^{123(45)}\right. \\
+ \text { perms }]+\mathcal{O}(\epsilon), \\
M_{6}^{N=8}\left(1^{-}, 2^{-}, 3^{+}, 4^{+}, 5^{+}, 6^{+}\right)=\frac{1}{8}\langle 12\rangle^{8}\left[h(1,\{2\}, 3) h(3,\{4,5,6\}, 1) \operatorname{tr}^{2}[123(4+5+6)] \mathcal{I}_{4}^{123(456)}\right. \\
+h(1,\{2,3\}, 4) h(4,\{5,6\}, 1) \operatorname{tr}^{2}[1(2+3) 4(5+6)] \mathcal{I}_{4}^{1(23) 4(56)} \\
+ \text { perms }]+\mathcal{O}(\epsilon),
\end{aligned}
\end{gathered}
$$

where the permutation sums are over all distinct permutations.

As a check, we have explicitly calculated the cuts of the $N=8$ MHV supergravity amplitudes up to six legs in $D=4$ (i.e., through $\mathcal{O}\left(\epsilon^{0}\right)$ ). We find complete agreement with the results (4.30) obtained via eq. (3.16). This cut calculation is similar to the one performed for the all-plus amplitudes in section 4.3, and makes use of the KLT relations (2.2) to express the the gravity tree amplitudes appearing in the cuts in terms of gauge theory amplitudes.

\subsection{Power-Counting for $N=8 \mathrm{MHV}$ Amplitudes vs. $N=4$}

Let us compare the structure of the $N=8 \mathrm{MHV}$ results (4.30) with general expectations from loopmomentum power-counting. First recall from section 3.4 that in a one-loop amplitude in $N=4$ super-Yang-Mills theory, a maximum of $m-4$ powers of loop momentum can appear in the numerator of each $m$-point integral. In a string-based approach $[56,50]$, the loop-momentum integrand for $N=8$ supergravity is just the product of two $N=4$ integrands. Therefore one expects a maximum of $2(m-4)$ powers of loop momentum to appear in the numerator of an $m$-point integral for $N=8$ supergravity. After carrying out the same integral reductions sketched in section 3.4, this powercounting allows for box integrals with up to $n-4$ powers of loop momentum in the numerator [57], for an $n$-point amplitude. Such integrals can be reduced to scalar box integrals, but (for $n \geq 5$ ) only at the expense of introducing scalar triangle and perhaps bubble integrals. On the other hand, we find no such integrals in eq. (4.30), only scalar box integrals. Nor will we find any need for integrals besides scalar boxes in the all- $n$ ansatz in section 6 .

In other words, all the $N=8 \mathrm{MHV}$ amplitudes are consistent with having at most $(m-4)$ powers of loop momentum for each $m$-point integral, instead of the $2(m-4)$ powers expected from 'squaring' gauge theory. This better-than-expected ultraviolet behavior can be contrasted with the recent analysis of multi-loop four-point amplitudes [6], in which the multi-loop $N=8$ amplitudes had exactly the same number of powers of loop momentum as expected from $N=4$ gauge theory. The additional cancellations we find in one-loop MHV amplitudes in $N=8$ supergravity amplitudes for $n \geq 5$ legs presumably arise from sums over different orderings of external legs. It would be interesting to know whether they can be understood at the Lagrangian level, or in a string-based framework, and whether they might extend to non-MHV helicity configurations as well, or to theories 
with less supersymmetry.

\section{Soft and Collinear Behavior of Gravity Amplitudes}

In order to extend the results of the previous section beyond the six-point level we will make use of the analytic behavior of gravity amplitudes as momenta become soft or collinear. A feature that the all-plus gravity and $N=8 \mathrm{MHV}$ amplitudes have in common with the all-plus gauge and $N=4$ MHV amplitudes is the absence of multi-particle kinematic poles. (This may be demonstrated using the SWI (2.4), which implies the vanishing of each product of amplitudes, tree $\times$ loop, that forms the residue of a multi-particle pole.) It is therefore sufficient to focus on the soft and collinear limits, which determine the two-particle poles. We perform our analysis in Minkowski space-time with signature $(1,3)$.

The behavior of tree-level gravity amplitudes as momenta become soft is well known [5, 4], and was reviewed in section 2.2. However, the behavior as momenta become collinear - outlined previously in ref. [14] — is more subtle. ${ }^{2}$ In the following subsection we obtain the graviton collinear splitting amplitudes from the gauge theory ones using the KLT expressions (2.2). We then show that these splitting amplitudes are universal: they apply to collinear limits of amplitudes with an arbitrary number of external legs. Furthermore, we shall argue in section 5.2 that the tree-level soft functions and collinear splitting amplitudes suffer no higher loop corrections. That is, we shall show that at any loop order (including tree level) a gravity amplitude behaves as

$$
M_{n}^{\text {loop }}\left(\ldots, a^{\lambda_{a}}, b^{\lambda_{b}}, \ldots\right) \stackrel{a \| b}{\longrightarrow} \sum_{\lambda} \operatorname{Split}_{-\lambda}^{\text {gravity }}\left(z, a^{\lambda_{a}}, b^{\lambda_{b}}\right) \times M_{n-1}^{\text {loop }}\left(\ldots, P^{\lambda}, \ldots\right),
$$

when $k_{a}$ and $k_{b}$ are collinear, and as

$$
M_{n}^{\text {loop }}\left(\ldots, a, s^{ \pm}, b, \ldots\right) \stackrel{k_{s} \rightarrow 0}{\longrightarrow} \mathcal{S}^{\text {gravity }}\left(s^{ \pm}\right) \times M_{n-1}^{\text {loop }}(\ldots, a, b, \ldots),
$$

when $k_{s}$ becomes soft.

\subsection{Collinear Behavior of Gravity from Gauge Theory}

Assuming that the collinear behavior of graviton amplitudes is universal, the splitting amplitudes in eq. (5.1) can be computed in terms of the gauge splitting amplitudes, using the four- and five-point KLT relations. Taking $1 \| 2$ in the five-point gravity amplitude (2.2) and applying eq. (2.17) we have,

$$
\begin{aligned}
\operatorname{Split}_{-(\lambda+\tilde{\lambda})}^{\text {gravity }}\left(z, 1^{\lambda_{1}+\tilde{\lambda}_{1}}, 2^{\lambda_{2}+\tilde{\lambda}_{2}}\right)=-s_{12} & \times \operatorname{Split}_{-\lambda}^{\mathrm{tree}}\left(z, 1^{\lambda_{1}}, 2^{\lambda_{2}}\right) \\
& \times \operatorname{Split}_{-\tilde{\lambda}}^{\mathrm{tree}}\left(z, 2^{\tilde{\lambda}_{2}}, 1^{\tilde{\lambda}_{1}}\right),
\end{aligned}
$$

where Split ${ }_{-\lambda}^{\text {gravity }}$ is a tree-level gravity splitting amplitude and the $\mathrm{Split}_{-\lambda}^{\text {tree }}$ are gauge theory splitting amplitudes, such as those given in eqs. (2.18) and (2.19).

\footnotetext{
${ }^{2}$ The suggestion that collinear limits in gravity are universal was made by Chalmers and Siegel [23].
} 
Equation (5.3) may be applied to arbitrary $N=8$ supergravity states by factorizing them into products of states in $N=4$ gauge theory, as discussed in section 2.1; the addition of helicities in the equation, $\lambda_{i}+\tilde{\lambda}_{i}$, corresponds to this factorization. For example, the pure graviton splitting amplitudes are obtained by substituting the values of the pure gluon splitting amplitudes (2.18) into eq. (5.3), yielding

$$
\begin{aligned}
& \operatorname{Split}_{+}^{\text {gravity }}\left(z, a^{+}, b^{+}\right)=0 \\
& \operatorname{Split}_{-}^{\text {gravity }}\left(z, a^{+}, b^{+}\right)=-\frac{1}{z(1-z)} \frac{[a b]}{\langle a b\rangle}, \\
& \operatorname{Split}_{+}^{\text {gravity }}\left(z, a^{-}, b^{+}\right)=-\frac{z^{3}}{1-z} \frac{[a b]}{\langle a b\rangle} .
\end{aligned}
$$

As a second example, the splitting amplitudes for a graviton into two gravitinos $\left(h^{+} \rightarrow \tilde{h}^{-} \tilde{h}^{+}\right)$follow from eqs. (5.3), (2.18) and (2.19),

$$
\operatorname{Split}_{+}^{\text {gravity }}\left(z, a_{\tilde{h}}^{-}, b_{\tilde{h}}^{+}\right)=-s_{a b} \times \operatorname{Split}_{+}^{\text {tree }}\left(z, a^{-}, b^{+}\right) \times \operatorname{Split}_{+}^{\text {tree }}\left(z, b_{q}^{+}, a_{\bar{q}}^{-}\right)=-\sqrt{\frac{z^{5}}{1-z}} \frac{[a b]}{\langle a b\rangle},
$$

and so forth.

In terms of its implication for subleading terms, eq. (5.1) has a slightly different meaning from the corresponding equations for the collinear limits in gauge theory, or for the soft limits in either gauge theory or gravity. In these other limits, the leading power-law behavior is determined; subleading, non-universal behavior is down by a power of either $\sqrt{k_{s}}$ or $\sqrt{s_{a b}}$. In the case of eq. (5.1), there are other terms of the same order as $[a b] /\langle a b\rangle$ as $s_{a b} \rightarrow 0$, namely any term that does not vanish as $s_{a b} \rightarrow 0$. However, these terms do not acquire any phase as $\vec{k}_{a}$ and $\vec{k}_{b}$ are rotated around their sum $\vec{P}$, as depicted in fig. 3, and thus they can be meaningfully separated from the terms described by eq. (5.1). (In space-time signature $(2,2)$, the spinor products $\langle a b\rangle$ and $[a b]$ are not complex conjugates of each other, so that $\langle a b\rangle$ can be taken to zero independently of $[a b]$, in order to separate out the $[a b] /\langle a b\rangle$ terms [23].)

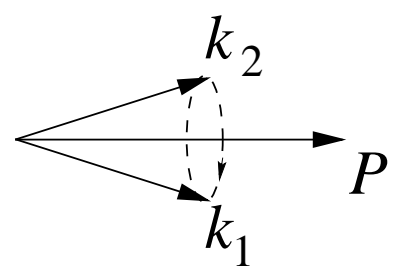

Figure 3: As two momenta become collinear the gravity $S$-matrix develops a phase singularity which can be detected by rotating the two momenta about the axis formed by their sum.

For example, consider the two factors,

$$
\begin{array}{ll}
\text { (a) } \frac{[12]}{\langle 12\rangle}, & \text { (b) } \frac{[13]}{\langle 13\rangle} \text {. }
\end{array}
$$

If we take $\vec{k}_{1}$ to be nearly collinear with $\vec{k}_{2}$ and rotate $\vec{k}_{1}$ and $\vec{k}_{2}$ around the vector $\vec{P}=\vec{k}_{1}+\vec{k}_{2}$ the factor (b) undergoes only a slight numerical variation. On the other hand, from eq. (2.7), the factor (a) undergoes a large phase variation, proportional to the angle of rotation. Thus a Fourier analysis 
in this azimuthal rotation angle will extract the universal terms in eq. (5.1) from the (approximately) constant non-universal terms, giving meaning to this equation.

The universality of the tree-level splitting amplitudes for gravity amplitudes with any number of external legs may be understood in terms of Feynman diagrams in any gauge which does not introduce extra singularities into the vertices or propagators, besides the usual $1 / p^{2}$ propagator factor (for example, de Donder gauge [58]). Although use of Feynman diagrams generally obscures the relationships between gravity and gauge theory scattering amplitudes, here we only require the diagrams' factorization properties. Terms with a phase singularity for $a \| b$ must contain a factor of the form $[a b]^{2} / s_{a b}$. The only tree-level Feynman diagrams that contain a pole in $s_{a b}$ (from a propagator) are of the type shown in fig. 4; they all contain the same three-point vertex. ${ }^{3}$ The splitting amplitudes are given by a straightforward evaluation of the three-vertex (multiplied by the $s_{a b}$ pole) in a helicity basis in the collinear limit. (For the analogous gauge theory computation, see refs. [31, 41].)

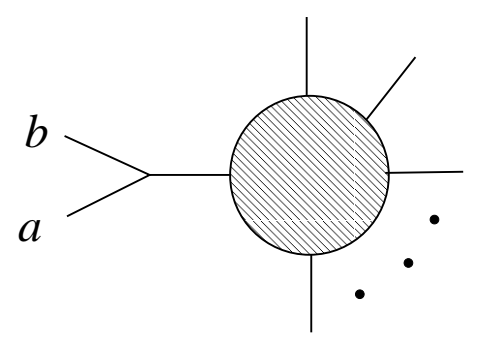

Figure 4: The class of tree diagrams in a gravity theory that can have a phase singularity factorizes in the collinear limit $a \| b$. The appearance of the same three-vertex for any number of external legs implies the universality of the tree-level splitting amplitudes. The splitting amplitudes are given by evaluating the three-vertex in the collinear limit in a helicity basis.

Similarly, the validity of the soft factor (2.21) for an arbitrary number of external legs also follows from the factorization properties of Feynman diagrams. In this case the tree diagrams that contribute to the soft factors are of the form shown in fig. 5, and the complete soft factor is given by summing over all three-point vertices with a soft leg (multiplied by the respective propagator).

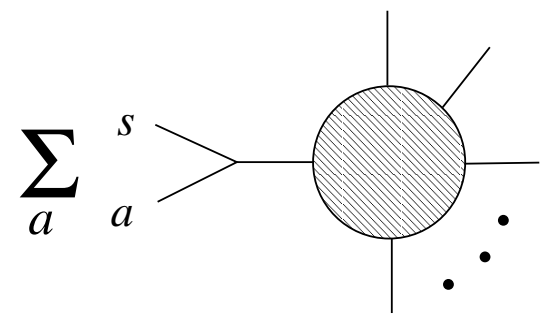

Figure 5: The class of tree diagrams in a gravity theory that contribute in the soft limit, where leg $s$ is soft. The soft functions are found by summing over all three-vertices containing a soft leg.

One may also prove the universality of the tree-level splitting amplitudes using the $n$-point version of the KLT relations given in appendix A. Alternatively, one may obtain the tree-level soft and collinear splitting functions from string theory by extending the gauge theory discussion given

\footnotetext{
${ }^{3}$ In the helicity formalism [29], a reference momentum entering the polarization vector or tensor could produce a pole in $s_{a b}$ in other diagrams, but this is easily avoided by choosing the reference momenta to be neither $k_{a}$ nor $k_{b}$.
} 
by Mangano and Parke [31] to the case of gravity, using a closed string instead of an open string. The factorization of the closed string integrands into products of open string integrands ensures that the gravity splitting functions are given in terms of products of the corresponding gauge theory splitting functions, as given in eq. (5.3).

\subsection{Absence of Loop Corrections}

We now show that the soft and collinear splitting amplitudes for gravity — in contrast to those for gauge theory - do not have any higher loop corrections. In general, in covariant gauges the splitting and soft functions may be classified into two categories: factorizing and non-factorizing contributions [41]. Diagrams for the factorizing one-loop corrections to the splitting and soft functions are shown in fig. 6. Non-factorizing contributions can arise whenever infrared divergences do not behave smoothly in the soft or collinear limits, as discussed in ref. [41].

First consider the factorizing contributions. Since each Feynman diagram in fig. 6 has a power of $\kappa^{2}$ as compared to the tree-level functions, dimensional analysis requires that diagram carries an extra power of $\left|s_{a b}\right|$ in the collinear case or an extra power of $\left|s_{a s}\right|$ in the soft limit. This suppresses potential one-loop corrections to either the collinear $\left(s_{a b} \rightarrow 0\right)$ or soft $\left(s_{a s} \rightarrow 0\right)$ limits.

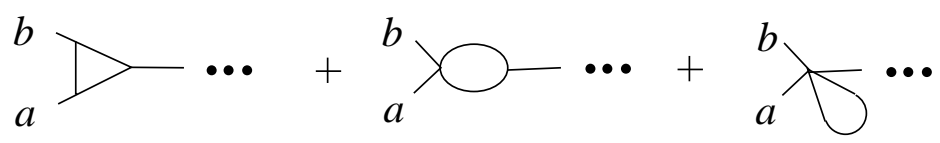

(a)

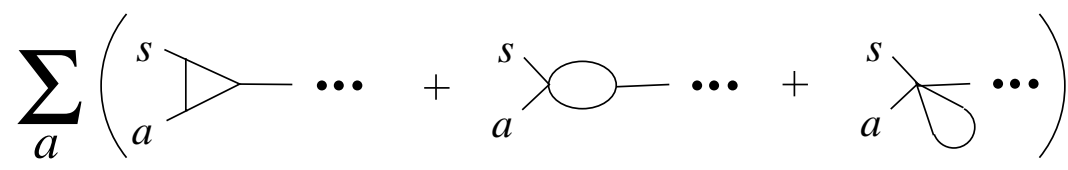

(b)

Figure 6: One-loop factorizing corrections to (a) the collinear splitting amplitudes and (b) the soft functions.

Now consider the non-factorizing contributions. In one-loop gauge theory amplitudes the infrared divergences, for e.g. a pure gluon amplitude, are of the form

$$
-A_{n}^{\text {tree }} \sum_{i \neq j}^{n}\left[\frac{1}{\epsilon^{2}}-\frac{\ln \left(-s_{i j}\right)}{\epsilon}\right] .
$$

The mismatch between the infrared divergence of the $n$ - and $(n-1)$-point one-loop amplitudes on the left- and right-hand-sides of the collinear limit (3.3) implies that there must be a non-trivial contribution to the one-loop gauge splitting amplitude. This may be contrasted with the case of gravity: a pure graviton amplitude has infrared divergences of the form [59]

$$
M_{n}^{\text {tree }} \sum_{i \neq j}^{n}\left[s_{i j} \frac{\ln \left(-s_{i j}\right)}{\epsilon}\right] .
$$

In this case the infrared divergences exhibit smooth behavior in soft or collinear limits, because of the extra power of $s_{i j}$ in each term; as any kinematic variable vanishes, the infrared divergent term 
containing that variable goes smoothly to zero. Thus there are no one-loop contributions to soft or collinear splitting amplitudes arising from non-factorizing contributions. Again this difference in behavior between the gauge and the gravity case is due to the dimensionful coupling in gravity theories.

More generally, the appearance of a dimensionful coupling in gravity implies that the contributions of the form that appear in gauge theory splitting amplitudes and soft functions will be suppressed by additional powers of vanishing $s_{i j}$ at all loop orders. For the factorizing contributions the argument is the same as for the one-loop case. For non-factorizing contributions, which involve infrared divergences, e.g. $\left(-s_{i j}\right)^{-\epsilon} / \epsilon^{2}$, a closer inspection is required.

In particular, in the gauge theory case it is possible for the loop integration to generate a pole in $s_{i j}$, leading to a non-factorizing contribution to soft or splitting amplitudes. A one-loop example of a diagram where this can happen is shown in fig. 7a. In the soft limit where $k_{1} \rightarrow 0$, the region of loop integration that can produce a kinematic pole in $k_{1}$ is where an extra propagator diverges.

As an example, before taking $k_{1} \rightarrow 0$, in the region $L_{1} \approx 0$ only the three propagators with momenta $L_{n}, L_{1}$ and $L_{2}$ in fig. 7 diverge. As $k_{1} \rightarrow 0$, the propagator with momentum $L_{3}=$ $L_{1}-k_{1}-k_{2}$ also diverges since $L_{3}^{2} \approx 2 k_{1} \cdot k_{2}-2 L_{1} \cdot\left(k_{1}+k_{2}\right)$. Since we are interested only in the leading behavior as $k_{1} \rightarrow 0$ we may set $L_{1}=0$ in the remaining part of the diagram effectively leaving only a box diagram to be analyzed.

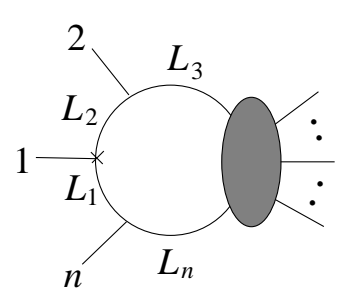

(a)

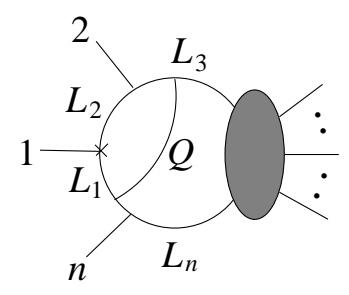

(b)

Figure 7: One- and two-loop examples of diagrams that can produce a pole in $k_{1}$ from the loop integration in the gauge theory case, but not in the gravity case.

For the case of gravity, the graviton vertex attached to leg 1 (and marked by a cross in the figure) contains one extra power of $k_{1}$ or $L_{1}$, as compared to the gauge theory vertex, and the contribution is therefore suppressed compared to gauge theory. Since one can obtain at most a single power of $1 / k_{1}$ in the gauge theory case, the gravity case cannot have a pole in $k_{1}$ and is therefore suppressed compared to the tree gravity soft $\mathcal{S}$ function which does contain a single pole in $k_{1}$. The cases where three propagators diverge can also be analyzed by observing that these cases effectively reduce to triangle integrals.

These arguments extend to the multi-loop case. Consider, for example, the two-loop diagram in fig. $7 \mathrm{~b}$. In the gauge theory case, in order to obtain a contribution analogous to the one-loop one discussed above we must also take $Q \approx 0$. Again the extra powers of $k_{1}, L_{1}$ or $Q$ in the vertices suppress any potential gravity contribution.

Similarly, for other potential non-factorizing soft contributions, and also in the case of collinear limits, one may show that the extra powers of momenta in the vertices suppress all potential loop 
contributions. Thus the appearance of a dimensionful coupling in gravity theories implies that the tree-level soft and collinear functions are exact to all orders of perturbation theory, so that eqs. (5.1) and (5.2) hold at any loop order.

\section{Ansatze for an Arbitrary Number of External Legs}

In this section we make use of the soft and collinear limits to construct ansatze for both the all-plus and $N=8 \mathrm{MHV}$ amplitudes for an arbitrary number of external legs. In a previous letter we presented the ansatz for the all-plus (self-dual) case [14]; here we provide some of the details of the derivation as well as an alternate representation of the amplitude that has manifest symmetry under relabelings of external legs. We also present a new ansatz for the $N=8$ supergravity amplitudes. The constraints that the amplitudes have the correct poles in all channels are rather restrictive and very likely determine the unique form of the amplitude, although we do not have a proof that this is so. Analogous constructions in the gauge theory case have been proven to lead to the correct results $[11,12,13]$.

\subsection{Functions with Simple Soft Properties}

The first step in constructing ansatze for the amplitudes is to find a set of functions which have simple behavior in the soft limits. A good starting point is the three $h$ functions defined in equations (4.16), (4.24) and (4.29), which appear in the coefficients of the box integrals in the explicit expressions for the four-, five- and six-point all-plus and $N=8 \mathrm{MHV}$ amplitudes. We collect them again here,

$$
\begin{aligned}
h(a,\{1\}, b) & =\frac{1}{\langle a 1\rangle^{2}\langle 1 b\rangle^{2}}, \\
h(a,\{1,2\}, b) & =\frac{[12]}{\langle 12\rangle\langle a 1\rangle\langle 1 b\rangle\langle a 2\rangle\langle 2 b\rangle}, \\
h(a,\{1,2,3\}, b) & =\frac{[12][23]}{\langle 12\rangle\langle 23\rangle\langle a 1\rangle\langle 1 b\rangle\langle a 3\rangle\langle 3 b\rangle}+\frac{[23][31]}{\langle 23\rangle\langle 31\rangle\langle a 2\rangle\langle 2 b\rangle\langle a 1\rangle\langle 1 b\rangle} \\
& \quad+\frac{[31][12]}{\langle 31\rangle\langle 12\rangle\langle a 3\rangle\langle 3 b\rangle\langle a 2\rangle\langle 2 b\rangle} .
\end{aligned}
$$

It is easy to verify that these functions satisfy the following soft limits,

$$
h(a, M, b) \stackrel{k_{m} \rightarrow 0}{\longrightarrow}-\mathcal{S}_{m}(a, M, b) \times h(a, M-m, b), \quad \text { for } m \in M .
$$

Here the 'half-soft' factor,

$$
\mathcal{S}_{m}(a, M, b) \equiv \frac{-1}{\langle a m\rangle\langle m b\rangle} \sum_{j \in M}\langle a j\rangle\langle j b\rangle \frac{[j m]}{\langle j m\rangle},
$$

is closely related to the gravity soft function $\mathcal{S}_{n} \equiv \mathcal{S}^{\text {gravity }}\left(n^{+}\right)$defined in eq. (2.21), except that the sum in eq. (6.2) is over only a subset of the legs in the amplitude. Thus $h$ obeys soft limits very similar to the tree-level gravity amplitudes, except that there is no momentum conservation 
constraint on $h$ or $\mathcal{S}_{m}$, so the $h$ functions may be thought of as off-shell extensions of the tree amplitudes.

Here we wish to find explicit forms for 'half-soft' functions $h(a, M, b)$, which satisfy eq. (6.2) for an arbitrary number of external legs. This is accomplished by using eq. (6.1) to motivate a guess for the general form of $h$, and then using the soft properties to fix its components. From the form of $\mathcal{S}_{m}(a, M, b)$, we see that $h(a, M, b)$ should be symmetric in $a \leftrightarrow b$, and in the exchange of any members of $M$. Also, eq. (6.1) suggests that it can be written as sums of products of spinor phase factors $\left[j_{1} j_{2}\right] /\left\langle j_{1} j_{2}\right\rangle$, where $j_{1}, j_{2} \in M$, multiplied by appropriate powers of $\left\langle a j_{l}\right\rangle\left\langle j_{l} b\right\rangle$. We write $h(a, M, b)$ as

$$
h(a,\{1,2, \ldots, m\}, b)=\sum_{i_{1}, i_{2}, \ldots, i_{m}=0}^{m-2} \phi\left(i_{1}, i_{2}, \ldots, i_{m}\right) \prod_{j=1}^{m}(\langle a j\rangle\langle j b\rangle)^{i_{j}-1},
$$

where $\phi\left(i_{1}, i_{2}, \ldots, i_{m}\right)$ is defined to be a symmetric function of its arguments, and nonzero only for $\sum_{j=1}^{m} i_{j}=m-2$. Thus at least one of the arguments $i_{j}$ must equal zero, and using the symmetry we can choose this to be the last argument. Then, to incorporate the soft limits (6.2), we define $\phi\left(i_{1}, i_{2}, \ldots, i_{m}\right)$ recursively by

$$
\begin{aligned}
\phi(0,0) & =\frac{[12]}{\langle 12\rangle}, \\
\phi\left(i_{1}, i_{2}, \ldots, i_{m-1}, 0\right) & =\sum_{j=1}^{m-1} \phi\left(i_{1}, i_{2}, \ldots, i_{j}-1, \ldots, i_{m-1}\right) \times \frac{[j m]}{\langle j m\rangle},
\end{aligned}
$$

where $\phi$ is also defined to be zero if any of its arguments is negative.

We give a few examples of the factors $\phi$ :

$$
\begin{aligned}
\phi(0,0) & =\frac{[12]}{\langle 12\rangle}, \\
\phi(1,0,0) & =\frac{[12][13]}{\langle 12\rangle\langle 13\rangle}, \\
\phi(2,0,0,0) & =\frac{[12][13][14]}{\langle 12\rangle\langle 13\rangle\langle 14\rangle}, \\
\phi(1,1,0,0) & =\frac{[12][23][14]}{\langle 12\rangle\langle 23\rangle\langle 14\rangle}+\frac{[12][13][24]}{\langle 12\rangle\langle 13\rangle\langle 24\rangle}, \\
\phi(2,1,0,0,0) & =\frac{[12][23][14][15]}{\langle 12\rangle\langle 23\rangle\langle 14\rangle\langle 15\rangle}+\frac{[12][13][24][15]}{\langle 12\rangle\langle 13\rangle\langle 24\rangle\langle 15\rangle}+\frac{[12][13][14][25]}{\langle 12\rangle\langle 13\rangle\langle 14\rangle\langle 25\rangle} .
\end{aligned}
$$

An interesting property of the $\phi$ functions is that they can be generated from group theory Young tableaux, as an alternative to eq. (6.5). We can restrict our attention to the $\phi\left(i_{1}, i_{2}, \ldots, i_{m}\right)$ with $i_{1} \geq i_{2} \geq \cdots \geq i_{m}=0$, since all other $\phi$ 's can be obtained by simple relabelings. The formula for $\phi$ can be schematically represented as

$$
\phi\left(i_{1}, i_{2}, \ldots, i_{m}\right)=\sum_{\text {NSYT }} \underbrace{\left(\frac{[]}{\langle\rangle}\right) \cdots\left(\frac{[]}{\langle\rangle}\right)}_{m-1 \text { terms }},
$$


where each term in the sum corresponds to a non-standard Young tableaux (NSYT). The NSYT are defined as all possible labelings from 1 to $m-2$ of the tableaux (with $i_{1}$ boxes in the first row, $i_{2}$ boxes in the second row, etc...), with the restriction of ascending order along rows. The standard requirement of descending order along columns is relaxed. The rule for constructing each phase factor in eq. (6.7) from the corresponding NSYT is best illustrated by an example. For $\phi(2,1,0,0,0)$, the three terms in eq. (6.6) correspond to these NSYT:

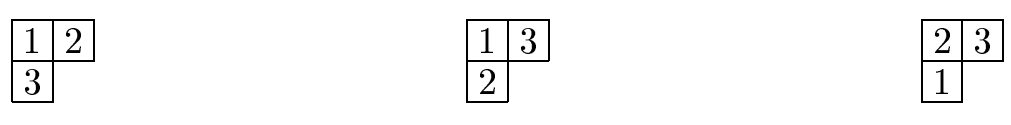

To obtain the phase factors, one first extends the YT vertically with 'empty' boxes until it has $m$ rows, in order to represent all $m$ arguments in $\phi$. Then one removes both the last empty box (in row $j$, say) and the full box containing the highest number (in row $i$ ), writing a factor of $[i j] /\langle i j\rangle$ for this step. Repeating the step until all boxes are gone yields the phase factor. For example,

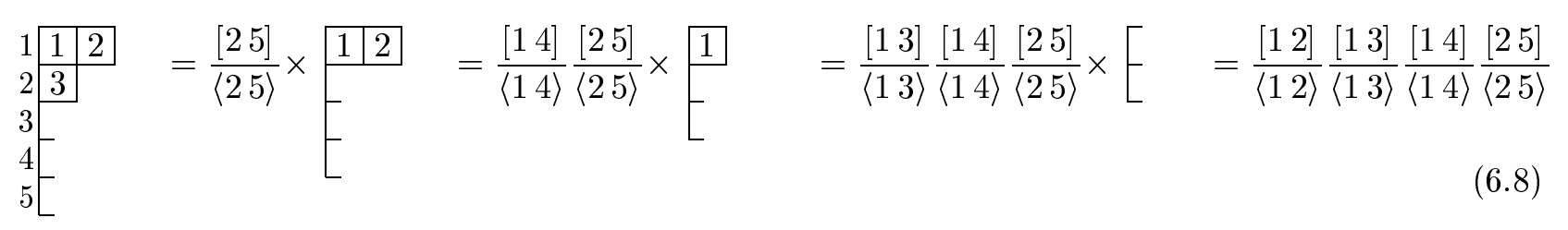

gives the last term in $\phi(2,1,0,0,0)$ in eq. (6.6).

The NSYT approach gives a simple formula for the number of terms in each $\phi$,

$$
\left[\# \text { of terms in } \phi\left(i_{1}, \ldots, i_{m}\right)\right]=\frac{(m-2) !}{\prod_{j=1}^{m} i_{j} !},
$$

which is the analog of the 'hook' formula for standard Young tableaux. The $\phi$ functions can also be generated graphically from certain 'elk diagrams', described in appendix C.2, which are in one-to-one correspondence with the NSYT.

Another approach to constructing the $h$ functions is the recursive currents method $[37,38,13]$ discussed in appendix B. Using this method, we have found an explicit non-recursive form for the functions,

$$
\begin{aligned}
h(a,\{1,2, \ldots, n\}, b) \equiv \frac{[12]}{\langle 12\rangle} \frac{\left\langle a^{-}\left|K_{1,2}\right| 3^{-}\right\rangle\left\langle a^{-}\left|K_{1,3}\right| 4^{-}\right\rangle \cdots\left\langle a^{-}\left|\not K_{1, n-1}\right| n^{-}\right\rangle}{\langle 23\rangle\langle 34\rangle \cdots\langle n-1, n\rangle\langle a 1\rangle\langle a 2\rangle\langle a 3\rangle \cdots\langle a n\rangle\langle 1 b\rangle\langle n b\rangle} \\
+\mathcal{P}(2,3, \ldots, n),
\end{aligned}
$$

where $K_{i, j}=k_{i}+k_{i+1}+\cdots+k_{j}$. In the form (6.10) the symmetry properties of $h$ under the interchange of $a \leftrightarrow b$ and $1 \leftrightarrow j \in\{2, \ldots, n\}$ are not manifest. Nevertheless, in appendix C.1 we show that the forms in eqs. (6.4) and (6.10) are in fact equal.

As mentioned above, the $h$ functions can be thought of as off-shell extensions of gravity tree amplitudes. Using the non-recursive form (6.10), it is not hard to show that they are related to the 
BGK expressions for the MHV tree amplitudes (2.14) via,

$$
\left.\frac{h(n,\{n-1, n-2, \ldots, 2\}, 1)}{\langle n 1\rangle^{2}}\right|_{k_{1}+k_{2}+\cdots+k_{n}=0}=(-1)^{n} \frac{M_{n}^{\text {tree }}\left(1^{-}, 2^{-}, 3^{+}, \ldots, n^{+}\right)}{i\langle 12\rangle^{8}} .
$$

In this form, momentum conservation only has to be used in one factor in $M_{n}^{\text {tree }}$, in order to convert it into $h$.

In light of eq. (6.11), it is perhaps not too surprising that the $h$ functions satisfy a squaring relation to the $g$ functions (3.14) appearing in the gauge theory amplitudes, analogous to the KLT relations for tree amplitudes. For example,

$$
\begin{aligned}
h(a,\{1\}, b) & =[g(a,\{1\}, b)]^{2}, \\
h(a,\{1,2\}, b) & =s_{12} g(a,\{1,2\}, b) g(a,\{2,1\}, b), \\
h(a,\{1,2,3\}, b) & =s_{12} s_{23} g(a,\{1,2,3\}, b) g(a,\{3,2,1\}, b)+\text { perms, }
\end{aligned}
$$

and so forth. These relations are analogous to the KLT relations, in eq. (2.2), except that they hold for functions that appear at one loop (and the $s_{i j}$ factors and permutations appearing are not precisely the same).

\subsection{Ansatz for All-Plus Amplitudes}

The forms of the four-, five- and six-point amplitudes in eqs. (4.15), (4.23) and (4.28), and the soft properties (6.2) of the $h$ functions have led us to the following ansatz (see also ref. [14]) for the one-loop all-plus (self-dual) amplitudes in $D=4$,

$$
M_{n}\left(1^{+}, 2^{+}, \ldots, n^{+}\right)=-\frac{i(-1)^{n}}{(4 \pi)^{2} \cdot 960} \sum_{\substack{1 \leq a<b \leq n \\ M, N}} h(a, M, b) h(b, N, a) \operatorname{tr}^{3}[a M b N]+\mathcal{O}(\epsilon),
$$

where $a$ and $b$ are massless legs, and $M$ and $N$ are two sets forming a 'distinct non-trivial partition' of the remaining $n-2$ legs; i.e., $M$ and $N$ should both be non-empty, and the partition $(M, N)$ is not considered distinct from $(N, M)$. This configuration of external legs is depicted in fig. 8. We do not have an ansatz that works to all orders in $\epsilon$. The $D=4$ amplitudes (6.13) are also generated by a self-dual gravity action $[60,22,23]$.

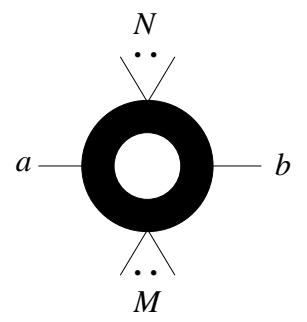

Figure 8: The configurations of external legs that are summed over in eq. (6.13).

The fact that the amplitudes (6.13) have the correct soft limits is a consequence of the soft properties of the $h$ functions, eq. (6.2). As $k_{n} \rightarrow 0$, the term labeled by $(a, M, b, N)$ in $M_{n-1}$ gets contributions from two terms in $M_{n}$, those labeled by $(a, M+n, b, N)$ and $(a, M, b, N+n)$. Each 
of the factors $h(a, M+n, b)$ and $h(b, N+n, a)$ in eq. (6.13) supplies 'half' of the soft factor in this limit, since

$$
\mathcal{S}_{n}=\mathcal{S}_{n}(a, M, b)+\mathcal{S}_{n}(b, N, a) .
$$

The trace factors behave smoothly in the soft limit, serving only to prevent the unwanted terms where $a$ or $b$ becomes soft.

The collinear properties are slightly more difficult to establish. They rely on the two non-trivial collinear limits of the half-soft function $h$ (after taking into account its symmetries),

$$
\begin{aligned}
h(a,\{1,2,3, \ldots, n\}, b) & \stackrel{1 \| 2}{\longrightarrow} \frac{1}{z(1-z)} \frac{[12]}{\langle 12\rangle} \times h(a,\{P, 3, \ldots, n\}, b), \\
\quad h(1,\{2,3, \ldots, n\}, b) & \stackrel{1 \| 2}{\longrightarrow} \frac{1}{\langle 12\rangle} \frac{\langle 1 b\rangle\left\langle b^{-}\left|K_{3, n}\right| 2^{-}\right\rangle}{\langle 2 b\rangle^{2}} \times h(1,\{3, \ldots, n\}, b),
\end{aligned}
$$

where we have used the Schouten identity, eq. (C.5), to derive the second limit, dropping terms without phase singularities, in accordance with the discussion in section 5.1.

Consider the collinear limit $1 \| 2$ of $M_{n}\left(1^{+}, 2^{+}, \ldots, n^{+}\right)$. For a term labeled by $(a, M, b, N)$ in the ansatz (6.13), there are three independent non-trivial possibilities:

(1) 1 and 2 both belong to the same set, say $M$,

(2) $a=1$ and $b \neq 2$, so that 2 belongs to a massive set,

(3) $a=1$ and $b=2$.

Case (3) is trivial; eq. (6.10) has no $1 /\langle a b\rangle$ factor, and hence there is no contribution to the collinear (phase) singularity. Case (1) is also simple. The first eq. (6.15) shows that these terms precisely account for all the terms in the expression (6.13) for the target amplitude $M_{n-1}\left(P^{+}, \ldots, n^{+}\right)$ in which $P \in M$.

The only remaining task is to show that the case (2) terms correctly give rise to the terms in the expression for the target amplitude in which $P$ does not belong to $M$ or $N$. The second eq. (6.15) shows that an individual $h$ function has a 'too singular' $1 /\langle 12\rangle$ behavior in case (2). However, the combination of two different terms, labeled by $(1, M+2, b, N)$ and $(1, M, b, N+2)$, cancels the singularity down to the desired level of $[12] /\langle 12\rangle$. More concretely, by using momentum conservation, $K_{M}+K_{N}+k_{1}+k_{2}+k_{b}=0$, where $K_{M}$ is the sum of the massless momenta in the set $M$, we have $\left\langle 1^{+}\left|K_{M}\right| b^{+}\right\rangle=-\left\langle 1^{+}\left|K_{N}\right| b^{+}\right\rangle-[12]\langle 2 b\rangle$. Also expanding the traces to first order in [1 2], we obtain

$$
\begin{aligned}
& h(1, M+2, b) h(b, N, 1) \operatorname{tr}^{3}[1(M+2) b N]+h(1, M, b) h(b, N+2,1) \operatorname{tr}^{3}[1 M b(N+2)] \\
& \stackrel{1 \| 2}{\longrightarrow} \frac{z^{3}+3 z^{2}(1-z)}{z(1-z)} \frac{[12]}{\langle 12\rangle} h(P, M, b) h(b, N, P) \operatorname{tr}^{3}[P M b N] .
\end{aligned}
$$

Adding the analogous equation with the roles of 1 and 2 (and $z$ and $1-z$ ) exchanged gives

$$
\begin{aligned}
& h(1, M+2, b) h(b, N, 1) \operatorname{tr}^{3}[1(M+2) b N]+h(1, M, b) h(b, N+2,1) \operatorname{tr}^{3}[1 M b(N+2)]+(1 \leftrightarrow 2) \\
& \stackrel{1 \| 2}{\longrightarrow} \frac{1}{z(1-z)} \frac{[12]}{\langle 12\rangle} h(P, M, b) h(b, N, P) \operatorname{tr}^{3}[P M b N],
\end{aligned}
$$

which accounts properly for the terms in the target expression $M_{n-1}$ in which $P=a$ (or similarly, $P=b)$. Thus $M_{n}\left(1^{+}, 2^{+}, \ldots, n^{+}\right)$does obey the required collinear limits. 


\subsection{Ansatz for $N=8 \mathrm{MHV}$ Amplitudes}

In section 4.4 we used the dimension-shifting relation (3.16) to obtain the four-, five- and six-point one-loop MHV amplitudes in $N=8$ supergravity, given in eq. (4.30), from the corresponding allplus amplitudes. These results suggest the following ansatz for the $n$-point one-loop MHV $N=8$ amplitudes:

$$
M_{n}^{N=8}\left(1^{-}, 2^{-}, 3^{+}, \ldots, n^{+}\right)=\frac{(-1)^{n}}{8}\langle 12\rangle^{8} \sum_{\substack{1 \leq a<b \leq n \\ M, N}} h(a, M, b) h(b, N, a) \operatorname{tr}^{2}[a M b N] \mathcal{I}_{4}^{a M b N}+\mathcal{O}(\epsilon),
$$

where the notation is identical to that of eq. (6.13). The scalar box integral functions, $\mathcal{I}_{4}^{a M b N}$, are given through $\mathcal{O}\left(\epsilon^{0}\right)$ by eq. (D.4). The sum in eq. (6.18) includes all inequivalent two-mass scalar box integrals with diagonally-opposite massive legs, as shown in fig. 1, as well as all one-mass scalar box integrals arising from the terms in eq. (6.18) where either $M$ or $N$ consists of a single massless leg. (In the four-point case the sum is over 6 boxes with all massless legs, of which only 3 are inequivalent because of an extra degeneracy, leading to the extra factor of 2 in the first line of eq. (4.30).) The $N=8$ SWI, eq. (2.10), requires that $M_{n}^{N=8}\left(1^{-}, 2^{-}, 3^{+}, \ldots, n^{+}\right) /\langle 12\rangle^{8}$ is totally symmetric with respect to permutations of its arguments; this symmetry is manifest in eq. (6.18).

Just as in the case of the all-plus amplitudes, the soft behavior of eq. (6.18) follows from the soft properties of the $h$ functions. The only real difference is that one of the powers of the trace $\operatorname{tr}[a M b N]$ is replaced by $\mathcal{I}_{4}^{a M b N}$. But these integrals also transform smoothly in the limit $k_{n} \rightarrow 0$,

$$
\mathcal{I}_{4}^{a M b(N+n)} \quad \text { and } \quad \mathcal{I}_{4}^{a(M+n) b N} \quad \stackrel{k_{n} \rightarrow 0}{\longrightarrow} \quad \mathcal{I}_{4}^{a M b N},
$$

and two powers of the traces suffice to kill the unwanted terms where either $a$ or $b$ becomes soft $\left(\mathcal{I}_{4}^{a M b N}\right.$ does not develop a singularity as $\left.k_{a} \rightarrow 0\right)$.

The ansatz (6.18) also must have universal collinear behavior. In the limit $1 \| 2$, the analysis is again quite similar to that presented in section 6.2 for the all-plus ansatz. The same three cases are encountered; the only subtle case is case (2). In this case, using the same labeling as in the all-plus discussion, the coefficients of the scalar box integrals $\mathcal{I}_{4}^{1(M+2) b N}, \mathcal{I}_{4}^{1 M b(N+2)}, \mathcal{I}_{4}^{2(M+1) b N}$ and $\mathcal{I}_{4}^{2 M b(N+1)}$ each have a $1 /\langle 12\rangle$ singularity. This singularity should cancel, since we only expect the phase singularity $[12] /\langle 12\rangle$. The cancellation can be demonstrated with the use of integral relations of the type,

$$
z \mathcal{I}_{4}^{1(M+2) b N}+(1-z) \mathcal{I}_{4}^{2 M b(N+1)} \stackrel{1 \| 2}{\longrightarrow} \mathcal{I}_{4}^{P M b N}+\mathcal{O}\left(\sqrt{s_{12}}\right)
$$

where $k_{1}=z k_{P}$ and $k_{2}=(1-z) k_{P}$ in the collinear limit. However, to verify that phase singularity of eq. (6.18) matches that of eq. (5.4) requires collinear analysis of the integrals to one higher order in $\sqrt{s_{12}}$. The required integral relation appears to be more subtle and involves a larger combination of integrals. We have, however, verified numerically that the ansatz (6.18) has the correct collinear limits for $n \leq 7$. (We have also checked numerically through $n=8$ that the infrared singularities of $M_{n}^{N=8}$ are correctly given by eq. (5.8).)

Comparing the all-plus and $N=8 \mathrm{MHV}$ ansatze, eqs. (6.13) and (6.18), it might appear that the former is obtained from the latter (up to an overall factor) simply by substituting the higherdimensional values (D.9) for the box integrals. However, the dimension-shifting relation does not 
work that simply, for $n=5$ or 6 . The quadratic polynomial for $\mathcal{I}_{4}^{a M b N}\left[\mu^{8}\right]$ in eq. (D.9) is not the same as the extra trace factor $\operatorname{tr}[a M b N]$ in the all-plus expression, and the pentagon and hexagon contributions to eq. (4.30) give a non-vanishing contribution, which somehow compensates for this discrepancy. Presumably the required rearrangements become yet more complicated for $n>6$.

It is instructive to compare the one-loop MHV $N=8$ supergravity amplitudes, eq. (6.18), with the corresponding amplitudes in $N=4$ super-Yang-Mills theory, eq. (3.11). Note that both amplitudes are expressed just in terms of scalar box integral functions. This result, though expected for $N=4$ super-Yang-Mills theory based on power-counting grounds, is somewhat surprising for $N=8$ supergravity, since as remarked in section 4.5 , a naive power-count for $n \geq 5$ does not exclude the appearance of triangle or bubble integrals.

\section{Discussion}

In this paper we have exploited relations between gauge theory and gravity to calculate the first three members of two infinite sequences of one-loop gravity amplitudes: the all-plus helicity amplitudes of non-supersymmetric gravity, and the maximally helicity-violating amplitudes of $N=8$ supergravity. From these results, and the analytic properties of $n$-graviton amplitudes, we obtained ansatze for the remaining members of both sequences.

In approaching any amplitude calculation in gravity we have found it useful to first consider the corresponding gauge theory calculation. Kawai, Lewellen and Tye (KLT) have given precise expressions for closed string tree amplitudes as (roughly speaking) the squares of open string tree amplitudes. In the field theory limit, this implies that properties of gauge theory tree amplitudes should be reflected in gravity tree amplitudes. As an example of this, we derived the properties of the gravity amplitudes as two momenta become collinear from the corresponding properties of gauge theory amplitudes.

The methods used to construct gravity amplitudes in this paper, relying on the analytic properties of the amplitudes, i.e. their (unitarity) cuts and poles, are essentially the same as those developed previously for evaluating multi-leg one-loop amplitudes in QCD [16, 51]. The analytic properties for dimensions away from $D=4$ are useful since they remove ambiguities normally associated with reconstruction of amplitudes from their cuts. When the KLT relations are combined with this analytic approach, gravity loop amplitudes can be obtained without evaluating even a single gravity Feynman diagram; the entire process relies only on gauge theory tree amplitudes.

Unitarity implies that relationships between tree amplitudes should somehow be reflected as relationships between loop amplitudes. Here we demonstrated that 'dimension-shifting' relations between one-loop all-plus and maximally helicity-violating $N=4$ supersymmetric gauge theory amplitudes can be transformed into relations between one-loop all-plus and maximally helicityviolating $N=8$ supersymmetric gravity amplitudes. The gravity relation is of practical value since it allows us to obtain the exact forms for the four-, five- and six-point $N=8$ amplitude without performing an explicit calculation; instead we applied a simple 'dimension shift' to the integral functions appearing in the all-plus amplitudes. We have also seen that there are 'squaring relations', 
eq. (6.12), reminiscent of the tree-level KLT relations, between the coefficients of the box integrals for $N=8$ supergravity and $N=4$ super-Yang-Mills, which have survived the integral reduction procedure.

The one-loop $N=8$ amplitudes first develop ultraviolet divergences in $D=8$. This is immediately apparent in the four-point amplitude first evaluated by Green, Schwarz and Brink [7]. In dimensional regularization, there are no divergences in odd dimensions, because odd-dimensional Lorentz-invariant quantities do not exist. So the next higher dimension one can inspect for one-loop ultraviolet divergences in $N=8$ supergravity is $D=10$. In the four-point amplitude, the $D=10$ divergence cancels [6], but from the point of view of eq. (4.12) the cancellation is again for a relatively trivial reason; the $D=10$ divergences of the sum of the three integrals has to be proportional to the only totally symmetric dimension-two four-point invariant, $s+t+u=0$. We have investigated the $D=10$ divergences for the five- and six-point amplitudes as well, using eq. (4.30), and find that they also cancel. (Because $D$ is not close to 4 , we need to use an expression good to all orders in $\epsilon$.) This cancellation may be connected with the fact that $D=10$ is the critical dimension for the superstring; however, it should be pointed out that the two-loop divergence does not cancel, even at the four-point level [6].

Currently the squaring relations that have been found between gravity and gauge theory are expressed entirely in terms of on-shell $S$-matrix elements. It would be nice to find an off-shell fieldtheoretic formulation of gravity that also exhibits a squaring relation with gauge theory, and to investigate the implications of such a formalism for the field equations.

\section{Acknowledgments}

We thank D.C. Dunbar, A.K. Grant and D.A. Kosower for helpful discussions.

\section{A KLT Relations for an Arbitrary Number of External Legs}

The KLT relations [1] between gravity and gauge amplitudes are, in the field theory limit and for an arbitrary number $n$ of external particles,

$$
\begin{aligned}
M_{n}^{\text {tree }}(1,2, \ldots, n)=i(-1)^{n+1} & {\left[A_{n}^{\text {tree }}(1,2, \ldots, n) \sum_{\text {perms }} f\left(i_{1}, \ldots, i_{j}\right) \bar{f}\left(l_{1}, \ldots, l_{j^{\prime}}\right)\right.} \\
& \left.\times A_{n}^{\text {tree }}\left(i_{1}, \ldots, i_{j}, 1, n-1, l_{1}, \ldots, l_{j^{\prime}}, n\right)\right] \\
& +\mathcal{P}(2, \ldots, n-2),
\end{aligned}
$$

where 'perms' are $\left(i_{1}, \ldots, i_{j}\right) \in \mathcal{P}(2, \ldots, n / 2),\left(l_{1}, \ldots, l_{j^{\prime}}\right) \in \mathcal{P}(n / 2+1, \ldots, n-2), j=n / 2-1$, $j^{\prime}=n / 2-2$, giving a total of $(n / 2-1) ! \times(n / 2-2)$ ! terms in the square brackets. We have assumed that $n$ is even here; the case of odd $n$ is completely analogous. The functions $f$ and $\bar{f}$ are given by

$$
\begin{aligned}
& f\left(i_{1}, \ldots, i_{j}\right)=s\left(1, i_{j}\right) \prod_{m=1}^{j-1}\left(s\left(1, i_{m}\right)+\sum_{k=m+1}^{j} g\left(i_{m}, i_{k}\right)\right), \\
& \bar{f}\left(l_{1}, \ldots, l_{j^{\prime}}\right)=s\left(l_{1}, n-1\right) \prod_{m=2}^{j^{\prime}}\left(s\left(l_{m}, n-1\right)+\sum_{k=1}^{m-1} g\left(l_{k}, l_{m}\right)\right),
\end{aligned}
$$


with

$$
g(i, j)= \begin{cases}s(i, j) \equiv s_{i j}, & i>j \\ 0, & \text { otherwise. }\end{cases}
$$

These definitions are used to compute the first term in the 'big' permutation sum; the rest of the terms $((n-3)$ ! all together $)$ are obtained by simply permuting the arguments of the $s(i, j)$ 's and

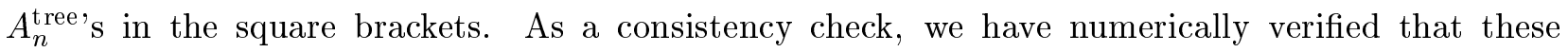
relations correctly give the MHV amplitudes of BGK, eq. (2.14), up to $n=8$.

\section{B Recursive Currents Approach}

The soft behavior of the half-soft functions $h(a, M, b)$ defined in section 6.1 is similar to that for a tree amplitude, except that the momenta belonging to $h(a, M, b)$ do not have to satisfy any momentumconservation relation. Thus we might suspect that the $h(a, M, b)$ could appear in off-shell quantities related to tree amplitudes.

\section{B.1 Off-Shell Currents $J$}

In gauge theory, Berends and Giele defined 'currents', sums of color-ordered Feynman diagrams where one leg is off-shell and the rest are on-shell [37]. These currents appear in recursive algorithms for generating multi-leg tree amplitudes. Mahlon used similar currents, but with two legs off-shell, to derive the one-loop gauge amplitudes $A_{n ; 1}\left(1^{ \pm}, 2^{+}, 3^{+}, \ldots, n^{+}\right)$[13]. In this appendix we discuss analogous currents in gravity, and show that the half-soft functions also appear there. The general factorization properties of such currents - which poles are present, with what residues, etc. — are very similar to the gauge case. This is because both tree-level gravity and gauge theory obey the supersymmetry Ward identities (2.4). However, the lack of color-ordering in the gravitational case leads to the appearance of more complicated structures. As in the gauge-theory case, the off-shell currents will depend on the choice of reference momenta for the on-shell gravitons.

We denote the generic gravitational current by $J^{\mu \alpha}(C)$, where the polarization tensor for the off-shell graviton leg is described by the index pair $\mu \alpha$, and $C \equiv\{1,2, \ldots, n\}$ stands for the set of on-shell gravitons, with momenta $k_{i}, i=1,2, \ldots, n$. By inspecting the tree-level Feynman diagrams contributing to $J^{\mu \alpha}(C)$, it is easy to derive the recursion relation,

$$
\begin{aligned}
J^{\mu \alpha}(C)=\frac{1}{K_{C}^{2}}\left\{\sum_{\substack{A \subset C \\
B \equiv C-A}} V_{3, \text { grav }}^{\mu \alpha, \nu \beta, \rho \gamma}\left(K_{A}, K_{B}\right) J^{\nu \beta}(A) J^{\rho \gamma}(B)\right. \\
\quad+\text { terms from 4- and higher-point graviton vertices }\},
\end{aligned}
$$

where $K_{A}^{\mu} \equiv \sum_{i \in A} k_{i}^{\mu}$. The sum here is over distinct partitions of the set $C$ into sets $A$ and $B$ (i.e. $B_{0}+A_{0}$ should not be counted in addition to $\left.A_{0}+B_{0}\right)$.

The three-graviton vertex $V_{3 \text {,grav }}$ is gauge-dependent in general. Although not essential, it is possible to choose a gauge [61] in which $V_{3 \text {,grav }}$ can be written as the square of the color-ordered 
gauge 3-vertex in Gervais-Neveu gauge [62], plus terms with the cyclic ordering reversed,

$$
\begin{aligned}
V_{3, \text { grav }}^{\mu \alpha, \nu \beta,}\left(K_{A}, K_{B}\right)= & -\frac{1}{2}\left[\left(\eta^{\rho \mu} K_{B}^{\nu}-\eta^{\mu \nu}\left(K_{A}+K_{B}\right)^{\rho}+\eta^{\nu \rho} K_{A}^{\mu}\right)\left(\eta^{\gamma \alpha} K_{B}^{\beta}-\eta^{\alpha \beta}\left(K_{A}+K_{B}\right)^{\gamma}+\eta^{\beta \gamma} K_{A}^{\alpha}\right)\right. \\
& \left.+\left(-\eta^{\rho \mu}\left(K_{A}+K_{B}\right)^{\nu}+\eta^{\mu \nu} K_{A}^{\rho}+\eta^{\nu \rho} K_{B}^{\mu}\right)\left(-\eta^{\gamma \alpha}\left(K_{A}+K_{B}\right)^{\beta}+\eta^{\alpha \beta} K_{A}^{\gamma}+\eta^{\beta \gamma} K_{B}^{\alpha}\right)\right]
\end{aligned}
$$

The currents we consider in the following will all satisfy the conservation law,

$$
K_{C}^{\mu} J^{\mu \alpha}(C)=K_{C}^{\alpha} J^{\mu \alpha}(C)=0 .
$$

In addition, they will vanish under the contractions,

$$
J^{\nu \beta}(A) J^{\nu \gamma}(B)=J^{\nu \beta}(A) J^{\gamma \nu}(B)=J^{\beta \nu}(A) J^{\gamma \nu}(B)=J^{\mu \mu}(A)=0 .
$$

For currents satisfying eq. (B.4), by using the fact that $m$-point vertices in gravity come from terms with two derivatives, i.e., $V_{m} \sim \partial^{2}(h . .)^{m}$, it is easy to see that only the 3 -vertex can contribute to eq. (B.1). Furthermore, the 3 -vertex can be simplified to

$$
V_{3, \text { grav,eff }}^{\mu \alpha, \nu \beta, \rho}\left(K_{A}, K_{B}\right)=-\left(\eta^{\rho \mu} K_{B}^{\nu}-\eta^{\mu \nu} K_{A}^{\rho}\right)\left(\eta^{\gamma \alpha} K_{B}^{\beta}-\eta^{\alpha \beta} K_{A}^{\gamma}\right)
$$

Define $J_{+q}^{\mu \alpha}(C)$ to be the all-plus current $J^{\mu \alpha}\left(1^{+}, 2^{+}, \ldots, n^{+}\right)$, where a common reference momentum $q$ is chosen for all the gravitons; that is, their polarization tensors in the spinor helicity formalism [29] are chosen to be

$$
\varepsilon_{+}^{\mu \alpha}(i) \equiv \frac{1}{2} \frac{\left\langle q^{-}\left|\gamma^{\mu}\right| i^{-}\right\rangle\left\langle q^{-}\left|\gamma^{\alpha}\right| i^{-}\right\rangle}{\langle i q\rangle^{2}}
$$

This reference momentum choice implies the vanishing of $\varepsilon^{\mu \alpha}(i) \varepsilon^{\mu \beta}(j)$ for all pairs $i, j$, which accounts for why eqs. (B.4) can be applied, and why 4- and higher-point vertices do not contribute in eq. (B.1).

Now, $J_{+q}^{\mu \alpha}$ contains no multi-particle poles, because of the SWI (2.4). Explicit computation gives for the first few $J_{+q}^{\mu \alpha}$,

$$
\begin{aligned}
J_{+q}^{\mu \alpha}\left(1^{+}\right) \equiv 2 \varepsilon_{+}^{\mu \alpha}(1)=\frac{\left\langle q^{-}\left|\gamma^{\mu}\right| 1^{-}\right\rangle\left\langle q^{-}\left|\gamma^{\alpha}\right| 1^{-}\right\rangle}{\langle 1 q\rangle^{2}}=\frac{\left\langle q^{-}\left|\gamma^{\mu} 1\right| q^{+}\right\rangle\left\langle q^{-}\left|\gamma^{\alpha} 1\right| q^{+}\right\rangle}{\langle 1 q\rangle^{4}}, \\
J_{+q}^{\mu \alpha}\left(1^{+}, 2^{+}\right)=\left\langle q^{-}\left|\gamma^{\mu}(1+2)\right| q^{+}\right\rangle\left\langle q^{-}\left|\gamma^{\alpha}(1+2)\right| q^{+}\right\rangle \times \frac{[12]}{\langle 1 q\rangle^{2}\langle 2 q\rangle^{2}\langle 12\rangle}, \\
J_{+q}^{\mu \alpha}\left(1^{+}, 2^{+}, 3^{+}\right)=\left\langle q^{-}\left|\gamma^{\mu}(1+2+3)\right| q^{+}\right\rangle\left\langle q^{-}\left|\gamma^{\alpha}(1+2+3)\right| q^{+}\right\rangle \\
\times\left(\frac{[12][13]}{\langle 2 q\rangle^{2}\langle 3 q\rangle^{2}\langle 12\rangle\langle 13\rangle}+\frac{[12][23]}{\langle 1 q\rangle^{2}\langle 3 q\rangle^{2}\langle 12\rangle\langle 23\rangle}+\frac{[13][23]}{\langle 1 q\rangle^{2}\langle 2 q\rangle^{2}\langle 13\rangle\langle 23\rangle}\right) .
\end{aligned}
$$

The result for $J_{+q}^{\mu \alpha}\left(1^{+}, 2^{+}, 3^{+}\right)$has been processed into this form using the knowledge that the $1 /\left(k_{1}+k_{2}+k_{3}\right)^{2}$ pole must cancel.

Comparing eq. (B.7) with the first few half-soft functions in eq. (6.1), it is clear that a specialized version of the half-soft coefficients appears in $J_{+q}^{\mu \alpha}(C)$, those where $a$ and $b$ are both equal. We are led to the ansatz

$$
J_{+q}^{\mu \alpha}(C)=\left\langle q^{-}\left|\gamma^{\mu} \not K_{C}\right| q^{+}\right\rangle\left\langle q^{-}\left|\gamma^{\alpha} \not_{C}\right| q^{+}\right\rangle \times h(q, C, q)
$$


Given this ansatz, eqs. (B.3) and (B.4) hold, and the recursion relation (B.1) reduces to a relation for $h$,

$$
\sum_{\substack{A \subset C \\ B \equiv C-A}} h(q, A, q) h(q, B, q)\left\langle q^{-}\left|K_{A} \not K_{B}\right| q^{+}\right\rangle^{2}=-K_{C}^{2} \times h(q, C, q) .
$$

In appendix C.2 we prove that the more general half-soft functions that actually appear in the one-loop amplitudes, namely $h(q, M, r)$ with $q \neq r$, obey an obvious extension of eq. (B.9),

$$
\sum_{\substack{A \subset C \\ B \equiv C-A}} h(q, A, r) h(q, B, r)\left\langle q^{-}\left|K_{A} \not K_{B}\right| q^{+}\right\rangle\left\langle r^{-}\left|K_{A} \not K_{B}\right| r^{+}\right\rangle=-K_{C}^{2} \times h(q, C, r) .
$$

This recursion relation is what one might expect for an all-plus off-shell current,

$$
J_{+q, r}^{\mu \alpha}(C) \equiv\left\langle q^{-}\left|\gamma^{\mu} K_{C}\right| q^{+}\right\rangle\left\langle r^{-}\left|\gamma^{\alpha} K_{C}\right| r^{+}\right\rangle \times h(q, C, r),
$$

corresponding to choosing graviton polarization tensors of the form

$$
\varepsilon_{+}^{\mu \alpha}(i) \equiv \frac{1}{2} \frac{\left\langle q^{-}\left|\gamma^{\mu}\right| i^{-}\right\rangle\left\langle r^{-}\left|\gamma^{\alpha}\right| i^{-}\right\rangle}{\langle i q\rangle\langle i r\rangle} .
$$

However, in contrast to the special case $q=r$, we have been unable to show for $q \neq r$ that eq. (B.10) actually follows from the vertices in the Einstein action. The difficulty is that for $q \neq r$ the 4 - and higher-point graviton vertices also contribute, at least in a general gauge, because eqs. (B.4) are no longer obeyed by the ansatz.

\section{B.2 Double Off-Shell Scalar Currents $S$}

We consider one additional tree-level off-shell structure, the doubly-off-shell scalar current $S_{q}(\ell ; C)$, which contains $n$ on-shell positive helicity gravitons for $C=\{1,2, \ldots, n\}$, plus a massless scalar line with outgoing momenta $\ell$ and $-\ell-K_{C}$ at the two ends of the line. By sewing the two off-shell ends of the scalar line to each other (in conjunction with an additional off-shell current), we can construct the one-loop all-plus gravity amplitudes $M_{n}(+,+, \ldots,+)$, in direct analogy to Mahlon's construction of the one-loop all-plus gauge amplitudes $A_{n ; 1}(+,+, \ldots,+)[13]$.

In practice, the one-loop expressions generated by this procedure are rather complicated (because of the high superficial degrees of divergence encountered), so in the end we have used them only as a numerical check. However, as a byproduct of calculating $S$ we have obtained a compact, non-recursive form for $h(q, M, r)$.

The recursion relation obeyed by $S_{q}$ is

$$
\begin{aligned}
S_{q}(\ell ; C) & =\frac{-1}{\left(\ell+K_{C}\right)^{2}} \sum_{\substack{A \subset C \\
B \equiv C-A}} S_{q}(\ell ; A)\left(\ell+K_{A}\right)^{\mu}\left(\ell+K_{A}\right)^{\alpha} J_{+q}^{\mu \alpha}(B) \\
& =\frac{-1}{\left(\ell+K_{C}\right)^{2}} \sum_{\substack{A \subset C \\
B \equiv C-A}} S_{q}(\ell ; A) h(q, B, q)\left\langle q^{-}\left|\left(\ell+K_{A}\right) \not K_{B}\right| q^{+}\right\rangle^{2},
\end{aligned}
$$

where the contribution of higher-point scalar+graviton vertices can be neglected using eq. (B.4). In eq. (B.13) the partition $B_{0}+A_{0}$ is distinct from $A_{0}+B_{0}$. Also, the set $A$ (but not $B$ ) is allowed to be the empty set \{\} (no graviton legs). The recursion is initiated by $S_{q}(\ell ;\{\})=1$. 
As with the current $J$, it is useful to generalize $S_{q}(\ell ; C)$ slightly to $S_{q, r}(L ; C)$, which is defined to obey

$$
S_{q, r}(L ; C)=\frac{-1}{\left(L+K_{C}\right)^{2}} \sum_{\substack{A \subset C \\ B \equiv C-A}} S_{q, r}(L ; A) h(q, B, r)\left\langle q^{-}\left|\left(\ell+\not K_{A}\right) \not K_{B}\right| q^{+}\right\rangle\left\langle r^{-}\left|\left(\ell+\not K_{A}\right) \not K_{B}\right| r^{+}\right\rangle,
$$

and $S_{q, r}(L ;\{\})=1$. Here $L$ is a $(4-2 \epsilon)$-dimensional momentum, with $L=\ell+\mu$ its decomposition into 4-dimensional and $(-2 \epsilon)$-dimensional pieces. We have found the following solution to the recursion relation,

$$
\begin{aligned}
S_{q, r}(L ; C)=L^{2} & \left\{\frac{1}{\langle 1 r\rangle\langle n r\rangle \prod_{j=1}^{n-1}\langle j, j+1\rangle} \prod_{j=1}^{n}\left(\frac{\left\langle q^{-}\left|\left(\ell+\not K_{1, j}\right)\right| j^{-}\right\rangle}{\langle q j\rangle}\right)\right. \\
& \times \sum_{m=1}^{n} \frac{1}{\left(L+K_{1, m-1}\right)^{2}\left(L+K_{1, m}\right)^{2}}\left[\left\langle r^{-}\left|\left(\ell+\not K_{1, m}\right) \not k_{m}\right| r^{+}\right\rangle-\mu^{2} \frac{\left\langle r^{-}\left|\not k_{m+1, n} \not k_{m}\right| r^{+}\right\rangle}{\left(L+K_{1, n}\right)^{2}}\right] \\
& +\mathcal{P}(1,2, \ldots, n)\} .
\end{aligned}
$$

Eq. (B.15) is not actually the full solution for arbitrary $\mu$, but rather the solution where the $\mu$ dependence is retained only in the last step of the recursion, and is dropped in previous steps. This approximation in $\mu$ turns out to be good enough to allow one to sew up $S_{q} \equiv S_{q, q}$ into the one-loop all-plus gravity amplitudes, $M_{n}(+,+, \ldots,+)$ (see below). The solution (B.15) was found by explicit calculation of the first few cases, making use of the factorization properties. (For example, for $\mu=0$, at most two different multi-particle poles can co-exist in a given term, and they should be of the form $1 /\left(L+K_{A}\right)^{2}$ and $1 /\left(L+K_{A}+k_{m}\right)^{2}$, where $A$ is some subset of $C$.) Eq. (B.15) has been checked directly up to $n=3$, and numerically for $\mu=0$ up through $n=8$; it also satisfies other consistency checks.

From the similarity between the recursion relations (B.10) and (B.14), it is easy to show that $h$ may be constructed from $S_{q, r}(L ; C)$ by taking the four-dimensional limit $(\mu \rightarrow 0, L \rightarrow \ell)$, and then the on-shell limit $\ell^{2} \rightarrow 0$,

$$
h(q,\{\ell, C\}, r)=\frac{1}{\langle\ell q\rangle^{2}\langle\ell r\rangle^{2}} \lim _{\substack{\ell^{2} \rightarrow 0 \\ \mu \rightarrow 0}} S_{q, r}(L ; C) .
$$

This limit gives a compact, non-recursive, form for $h$, to be contrasted with the recursive construction in section 6.1 . We get

$$
\begin{aligned}
h(q,\{1,2, \ldots, n\}, r) \equiv & \frac{[12]}{\langle 12\rangle} \frac{\left\langle q^{-}\left|K_{1,2}\right| 3^{-}\right\rangle\left\langle q^{-}\left|K_{1,3}\right| 4^{-}\right\rangle \cdots\left\langle q^{-}\left|K_{1, n-1}\right| n^{-}\right\rangle}{\langle 23\rangle\langle 34\rangle \cdots\langle n-1, n\rangle\langle q 1\rangle\langle q 2\rangle\langle q 3\rangle \cdots\langle q n\rangle\langle 1 r\rangle\langle n r\rangle} \\
& +\mathcal{P}(2,3, \ldots, n) .
\end{aligned}
$$

Although this expression is not manifestly symmetric in $q \leftrightarrow r$ or in $1 \leftrightarrow j \in\{2,3, \ldots, n\}$, in appendix C.1 we show that eq. (B.17) is equivalent to the recursive form (6.4) in which these symmetries are manifest. 


\section{B.3 Sewing of $S$ and $J$ to Obtain a Loop Amplitude}

Because the current $S_{q}$ contains two off-shell ends of a scalar line, one should be able to equate the momenta of the two ends, and integrate over $L$, in order to obtain the scalar loop contribution to $M_{n}(+,+, \ldots,+)$. Using the SWI $(2.4)$, the scalar contribution is the same, up to trivial overall factors, as the contribution of a fermion or a graviton in the loop. There are two subtleties:

(1) To avoid double-counting problems, one has to insert an additional current $J$ into the sewing operation. That is, one focuses on a particular leg, say $n$, and defines the tree by which it is attached to the loop to be $J^{\mu \alpha}(B)$ for some subset $B$, as depicted in fig. 9. The graviton legs not belonging to $B$ then belong to the complementary set $A \equiv C-B$, where $C=\{1,2, \ldots, n\}$. They can all be assigned to the double-off-shell scalar current $S_{q}(L ; A)$. Thus we obtain, after substituting in eq. (B.8) for $J_{+q}^{\mu \alpha}$,

$$
M_{n}\left(1^{+}, 2^{+}, \ldots, n^{+}\right)=\int \frac{d^{D} L}{(2 \pi)^{D}} \sum_{\substack{B \subset C ; n \in B \\ A \equiv C-B}} \frac{S_{q}(L ; A)}{L^{2}}\left\langle q^{-}\left|\ell K_{B}\right| q^{+}\right\rangle^{2} h(q, B, q) .
$$

(2) As explained by Mahlon [13] for the analogous gauge-theory case, one cannot set $D=4$ (i.e., $\mu=$ $0)$ immediately in eq. (B.18). Even though the loop amplitudes $M_{n}\left(1^{+}, 2^{+}, \ldots, n^{+}\right)$are ultraviolet finite, they are not finite term-by-term in the form given by eq. (B.18). Indeed, the $\mu$-independent terms in $S_{q}(L ; C)$ (eq. (B.15)) give a vanishing contribution: They each have only two propagators, separated by a single external leg. For these terms, the loop integral in eq. (B.18) becomes a two-point (bubble) integral with a massless external momentum, which is zero in dimensional regularization.

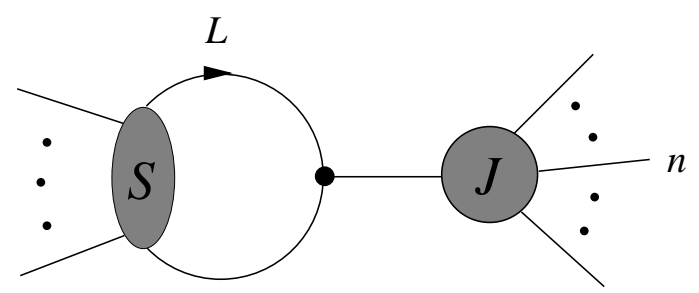

Figure 9: Sewing of a double-off-shell current $S$ and a single-off-shell current $J$ to obtain the all-plus loop amplitude $M_{n}\left(1^{+}, 2^{+}, \ldots, n^{+}\right)$. The particle circulating in the loop is a scalar, while the remaining lines are gravitons. The $n$th leg must belong to $J$.

Thus we have to retain the $\mu$-dependence in the last step of the recursion relation for $S_{q}$. The $\mu^{2}$ terms in eq. (B.15) have an additional propagator, so they correspond to a triangle integral in $D=6-2 \epsilon$, multiplied by a factor of $-\epsilon$. Because such a triangle integral has an ultraviolet $1 / \epsilon$ pole, we get a finite contribution as $\epsilon \rightarrow 0$. The reason one does not have to retain $\mu$-dependence for more than the last step of the $S_{q}$ recursion is related to the finiteness of the triangle integral in $D=4[13]$.

It is important to note in the preceding arguments that every loop momentum appears in a spinor string $\left\langle q^{-} \mid \ell \ldots\right\rangle$. Normally, contraction terms between different loop momenta in the numerator ( $\ell^{\mu} \ell^{\nu} \rightarrow \ell^{2} \delta^{\mu \nu}$, etc.) give the leading ultraviolet behavior. Here, however, a Fierz identity implies that all such contraction terms vanish:

$$
\left\langle q^{-} \mid \gamma^{\mu} \ldots\right\rangle\left\langle q^{-} \mid \gamma_{\mu} \ldots\right\rangle=2\langle q q\rangle\langle\ldots\rangle=0 .
$$


The surviving terms in the triangle integrals are those obtained by shifting the loop momenta by the usual amount depending on the Feynman parameters.

We have evaluated the relevant Feynman-parametrized triangle integrals as $\epsilon \rightarrow 0$ for both $M_{4}$ and $M_{5}$. The analytic expressions obtained are rather complicated; in particular the answers are not manifestly independent of the reference momentum $q$. However, numerical evaluation shows that both answers are indeed independent of $q$, and that they agree with the $D=4$ limits of $M_{4}$ and $M_{5}$ as obtained via cuts, eqs. (4.15) and (4.23).

We have carried out a similar numerical check for $n=4$, in the case that $q \neq r$, using $J_{+q, r}^{\mu \alpha}$ and $S_{q, r}$. This check is more complicated because: (a) there are a number of contraction terms now, proportional to $\langle q r\rangle$; (b) because of this, the $\mu^{2}$ approximation given for $S_{q, r}$ in eq. (B.15) is inadequate; and (c) also because of this, box integrals as well as triangle integrals have to be evaluated. Nevertheless, after making all three modifications the check works, which provides some evidence that the $q \neq r$ recursion relations we have used for $h(q, M, r)$ and $S_{q, r}$, eqs. (B.10) and (B.14), do actually follow from the Einstein action (in some gauge).

\section{Properties of the Half-Soft Functions}

\section{C.1 Equivalence of the Two Forms of $h(q, M, r)$}

In this appendix, we shall show that the non-recursive formula (B.17) (or eq. (6.10)) for the halfsoft functions $h(q, M, r)$, where $M=\{1,2, \ldots, n\}$, is equivalent to the recursive definition (6.4) in section 6.1. In the recursive solution, $h(q, M, r)$ is represented as a sum of terms of the form,

$$
\frac{[X]}{\langle X\rangle} \prod_{j=1}^{n}(\langle q j\rangle\langle j r\rangle)^{i_{j}-1}
$$

where $[X] /\langle X\rangle$ is one of the terms in the sum for the factor $\phi\left(i_{1}, i_{2}, \ldots, i_{n}\right)$. That is, $[X]$ is a product (obeying certain rules) of $n-1$ square brackets $[j k]$, with $j, k \in M ;\langle X\rangle$ is the product of the corresponding angle brackets; and $i_{j}=$ (number of appearances of $j$ in $\left.[X]\right)-1$. Through the recursive definition (6.5) of the $\phi$ 's, each term (C.1) can be built recursively, starting from

$$
\phi(0,0) \frac{1}{\langle q 1\rangle\langle 1 r\rangle} \frac{1}{\langle q 2\rangle\langle 2 r\rangle}=\frac{[12]}{\langle 12\rangle} \frac{1}{\langle q 1\rangle\langle 1 r\rangle} \frac{1}{\langle q 2\rangle\langle 2 r\rangle},
$$

and multiplying by

$$
\frac{[m l]}{\langle m l\rangle} \frac{\langle q m\rangle}{\langle q l\rangle} \frac{\langle m r\rangle}{\langle l r\rangle}
$$

at each step of the recursion, where $m$ is one of the 'legs' added in a previous step, and $l$ is the new 'leg' we add at this step.

Now, let us look at eq. (B.17). In each of the $(n-1)$ ! terms in the permutation sum we can expand each factor,

$$
\left\langle q^{-}\left|K_{1, l-1}\right| l^{-}\right\rangle=\sum_{j=1}^{l-1}\langle q j\rangle[j l]=\langle q 1\rangle[1 l]+\langle q 2\rangle[2 l]+\cdots+\langle q, l-1\rangle[l-1, l],
$$


and collect all terms having a given product of $(n-1)$ square brackets, which we shall also call $[X]$. We wish to show that

(a) the types of square bracket products $[X]$ that appear here are the same as in the recursive construction, and

(b) the products of angle bracket factors multiplying them are also the same.

To prove part (b) we will have to apply the Schouten identity on angle brackets,

$$
\langle i j\rangle\langle k l\rangle=\langle i k\rangle\langle j l\rangle+\langle i l\rangle\langle k j\rangle \text {. }
$$

We will not need the corresponding identity for square brackets; i.e. the square brackets are already in the correct form. Our strategy for part (b) will be a recursive one, based on demonstrating that the multiplicative factor (C.3) arises in going from $n-1$ to $n$.

First we establish part (a). Note from eq. (B.17) that each $j \in M$ (for $j \neq 1$ ) appears in $[X]$ once 'from the back' (i.e. from $\left\langle q^{-} \mid K_{\ldots} . j^{-}\right\rangle$), and the remaining $i_{j} \geq 0$ times 'from the front' (i.e. from $\left.\left\langle q^{-}\left|\ldots+\not k_{j}+\ldots\right| l^{-}\right\rangle\right)$. Because there are $n-1$ square brackets in $[X]$, each containing two arguments, there are a total of $2(n-1)=\sum_{j=1}^{n}\left(i_{j}+1\right)$ arguments in $[X]$, and we have $\sum_{j=1}^{n} i_{j}=n-2$, just as in the recursive formula. Since all the $i_{j}$ are non-negative, at least two of the $i_{j}$ must vanish. Thus we can always find some $l$, with $l \neq 1$, such that $i_{l}=0$; i.e. $l$ appears exactly once in $[X]$, from the back. It appears in the factor $[m l]$, for some $m \neq l$. Consider the product $[X]$ with $[m l]$ removed from it, which no longer contains $l$. That is, define $[\tilde{X}] \equiv[X] /[m l]$, and for $j \neq l$ let $\tilde{i}_{j}=$ (number of appearances of $j$ in $[\tilde{X}]$ ) -1 . We see that $[\tilde{X}]$ obeys the same 'counting rules' as $[X]$, except with $n-1$ arguments, because $l$ is no longer present: $\sum_{j \neq l} \tilde{i}_{j}=n-3$, with all $\tilde{i}_{j} \geq 0$, and each $j \neq l(j \neq 1)$ still appears at least once from the back. Hence we can again find some $l^{\prime}$, with $l^{\prime} \neq 1$, such that $\tilde{i}_{l^{\prime}}=0$; i.e. $l^{\prime}$ appears exactly once in $[\tilde{X}]$, from the back. Clearly, this procedure can be repeated until we have only two arguments left, at which stage we obtain the square bracket structure $[1 j]$ corresponding to $\phi(0,0)$. We have thus established part (a) by working backwards through the recursive construction of the $\phi$ factors, eq. (6.5).

To prove part (b), first note that the $\langle q j\rangle$ factors in the non-recursive formula already agree precisely with the recursive form, in every contributing permutation. As noted above, in the numerator of each term in eq. (B.17) each $j$ appears once from the back and $i_{j}$ times from the front. (For $j=1$, we count its appearance in [12] as 'from the back'.) In the second case, it is always accompanied by $\langle q j\rangle$, so we get $\langle q j\rangle^{i_{j}}$. But we also have $\langle q j\rangle$ in the denominator for each $j$, so overall we get $\langle q j\rangle^{i_{j}-1}$, as required by the recursive eq. (6.4). Thus we only have to rearrange the spinor products of the form $\langle j k\rangle$ and $\langle k r\rangle$, where $j, k \in M$.

In general, an individual term in the permutation sum for $h(q, M, r)$ in eq. (B.17) either contains the square bracket structure $[X]$ once, or does not contain it at all. (The fact that $j<l$ in eq. (C.4) means that, for a given permutation, one can uniquely determine which of the arguments in $[X]$ come from the front, and which from the back.) However, $[X]$ can appear in many different terms in the sum over $(n-1)$ ! permutations. The coefficient of

$$
[X] \prod_{j=1}^{n}\langle q j\rangle^{i_{j}-1}
$$


is

$$
\frac{1}{\langle 1 r\rangle} \sum_{\sigma \in \mathcal{P}_{[X]}} \frac{1}{\left\langle 1, \sigma_{2} \ldots \sigma_{n}, r\right\rangle}
$$

where the sum is over permutations $\sigma$ containing $[X],\left\{\sigma_{2} \ldots \sigma_{n}\right\}$ is a permutation of $\{2 \ldots n\}$, and

$$
\langle a, b c \ldots d, e\rangle \equiv\langle a b\rangle\langle b c\rangle \cdots\langle d e\rangle
$$

As in the proof of $(\mathrm{a})$, let $l \neq 1$ be the leg appearing exactly once in $[X]$, in the combination $[m l]$. Since $l$ appears from the back, only the permutations where $l$ appears after $m$ will contribute. In the permutation sum in eq. (C.7), let us hold fixed the positions of all legs except $l$, and just sum over the insertions of $l$ after $m$. From the Schouten identity (C.5) it is easy to derive the 'eikonal' identity

$$
\sum_{i=m}^{r-1} \frac{\langle i, i+1\rangle}{\langle i l\rangle\langle l, i+1\rangle}=\frac{\langle m r\rangle}{\langle m l\rangle\langle l r\rangle} .
$$

This identity allows us to simplify the sum over $l$ insertions,

$$
\sum_{\substack{l \text { after } m \\ \sigma_{2} \ldots \hat{l} \ldots \sigma_{n} \text { fixed }}} \frac{1}{\left\langle 1, \sigma_{2} \ldots l \ldots \sigma_{n}, r\right\rangle}=\frac{\langle m r\rangle}{\langle m l\rangle\langle l r\rangle} \frac{1}{\left\langle 1, \sigma_{2} \ldots \hat{l} \ldots \sigma_{n}, r\right\rangle},
$$

where a hat over $l$ signifies that it is no longer present.

We find that the terms in eq. (B.17) containing $[X]$ can be rewritten in terms of $[\tilde{X}] \equiv[X] /[m l]$ and $\tilde{i}_{j}$ as,

$$
\frac{[m l]}{\langle m l\rangle} \frac{\langle q m\rangle}{\langle q l\rangle} \frac{\langle m r\rangle}{\langle l r\rangle} \times[\tilde{X}] \prod_{\substack{j=1 \\ j \neq l}}^{n}\langle q j\rangle^{\tilde{i}_{j}-1} \times \frac{1}{\langle 1 r\rangle} \sum_{\sigma \in \mathcal{P}_{[\tilde{X}]}} \frac{1}{\left\langle 1, \sigma_{2} \ldots \hat{l} \ldots \sigma_{n}, r\right\rangle} .
$$

Note that the first factor is exactly the desired factor (C.3) appearing in the recursion relation when $l$ is added, and that the third factor is precisely the same as eq. (C.7) with $l$ removed. As in the proof of part (a), we can repeat this argument for $[\tilde{X}] /\left[m^{\prime} l^{\prime}\right]$, etc., until we arrive at the case $n=2$, which works by inspection. Thus we have proven part (b), by showing that the coefficient of $[X]$ is correct, inductively in $n$.

\section{C.2 Proof of $h(q, M, r)$ Recursion Relation}

In this appendix, we prove that the recursion relation (B.10) is satisfied by the $h(q, M, r)$. The key observation is (as in appendix C.1) that we do not need to use any Schouten identities on the square brackets (after we expand both sides so that they only contain square and angle brackets). So, our strategy will be to look at the coefficients of a given square bracket structure on both sides of eq. (B.10) and show that they are equal.

First we need to identify which square bracket structures can appear. The easiest way to do this is by representing such structures graphically. For each structure, we draw an 'elk diagram', according to following rules:

(a) Each element of $M$ corresponds to a node on the diagram.

(b) If the structure contains $[i j]$, draw a line connecting nodes $i$ and $j$. 
Each elk diagram corresponds to one of the non-standard Young tableaux discussed in section 6.1. The recursive construction of the square bracket structures in $\phi$, eq. (6.5), always adds a new node at each step. Therefore every elk diagram corresponding to a square bracket structure for $h(q, M, r)$ is a tree graph, with at least one line attached to each node.

Now look at the right-hand side of eq. (B.10). Any square bracket structure appearing there is obtained by multiplying some structure from $h(q, C, r)$ by one extra square bracket from $-K_{C}^{2}=$ $\sum_{i<j \in C}\langle i j\rangle[i j]$. The corresponding elk diagram is obtained by adding one line to the tree-like elk diagram for $h$. The new diagram must have one, and only one, closed loop. Thus the new square bracket structure will have exactly one closed cycle. There are two possible cases:

(i) The closed cycle consists of just two square brackets, $[i j]^{2}$. In this case, it is clear that one of the $[i j]$ 's comes from $K_{C}^{2}$, while the other one comes from $h$. The coefficient of a structure of this type consists of a single term, which is:

$$
\frac{[X]}{\langle X\rangle} \frac{[i j]}{\langle i j\rangle} \times\langle i j\rangle^{2} \times \prod_{k=1}^{n}(\langle q k\rangle\langle k r\rangle)^{i_{k}-1}
$$

where $[X]$ comes from $h$ (and contains $[i j]$ ), and $i_{k}=$ (number of appearances of $k$ in $X$ ) -1 .

(ii) The closed cycle contains several square brackets, e.g. $[[1 \ldots k]] \equiv[12][23] \ldots[k-1, k][k 1]$. In this case, it is not possible to identify uniquely which of the square brackets in the cycle came from $K_{C}^{2}$. The coefficient of such a structure will contain $k$ terms. It is equal to:

$$
\frac{[[1 \ldots k]]}{\langle\langle 1 \ldots k\rangle\rangle} \times\left(\sum_{[i j] \in \text { cycle }} \frac{\langle i j\rangle^{2}}{\langle q i\rangle\langle i r\rangle\langle q j\rangle\langle j r\rangle}\right) \times \frac{[Y]}{\langle Y\rangle} \prod_{m}(\langle q m\rangle\langle m r\rangle)^{i_{m}-1} \times \prod_{m \in \text { cycle }}(\langle q m\rangle\langle m r\rangle)^{2},
$$

where $[Y]$ are the square brackets in $[X]$ that $d o$ not enter the closed cycle, and $i_{m}=$ (number of appearances of $m$ in $Y$ ) -1 .

Next look at the left-hand side of eq. (B.10). First, consider square bracket structures of type (i). Note that only the terms with $i \in A, j \in B$ contribute, and both [ij]'s must come from $\left\langle q^{-}\left|K_{A} K_{B}\right| q^{+}\right\rangle\left\langle r^{-}\left|K_{A} \not K_{B}\right| r^{+}\right\rangle$, while the $h$ 's give rise to $[\hat{X}] \equiv[X] /[i j]$. There is only one such term. This is again easily seen using the elk diagram: cutting the 'double link' $(i j)$ separates the original diagram into two unique tree structures. Picking out the terms in $h(q, A, r)$ and $h(q, B, r)$ that contain these structures, we get:

$$
\frac{[\hat{X}]}{\langle\hat{X}\rangle} \times \frac{[i j]^{2}}{\langle i j\rangle^{2}} \times\langle i j\rangle^{2} \times\langle q i\rangle\langle i r\rangle\langle q j\rangle\langle j r\rangle \times \prod_{l}(\langle q l\rangle\langle l r\rangle)^{i_{l}-1},
$$

where $i_{l}=($ number of appearances of $l$ in $\hat{X})-1$. This is precisely eq. (C.12).

Finally, consider square bracket structures of type (ii). Here, the square brackets coming from $\left\langle q^{-}\left|K_{A} K_{B}\right| q^{+}\right\rangle$and $\left\langle r^{-}\left|K_{A} \not K_{B}\right| r^{+}\right\rangle$must belong to the cycle. Suppose these square brackets are $[i j]$ and $[k l]$. If we cut the links $(i j)$ and $(k l)$ in the original elk diagram, we separate it into two tree-like diagrams; we can then pick up the unique term in $h(q, A, r) h(q, B, r)$ which gives rise to these structures. To get the full coefficient of the original square bracket structure, we need to sum over various combinations of $(i j)$ and $(k l) \neq(i j)$ :

$$
\frac{[[1 \ldots k]]}{\langle\langle 1 \ldots k\rangle\rangle} \times\left(\sum_{(i j) \neq(k l)} \frac{\langle i j\rangle\langle k l\rangle}{\langle r i\rangle\langle r j\rangle\langle q k\rangle\langle q l\rangle}\right) \times \frac{[Y]}{\langle Y\rangle} \prod_{m}(\langle q m\rangle\langle m r\rangle)^{i_{m}-1} \times \prod_{m \in \text { cycle }}(\langle q m\rangle\langle m r\rangle)^{2} .
$$


Using the eikonal identity,

$$
\sum_{(k l) \neq(i j)} \frac{\langle k l\rangle}{\langle q k\rangle\langle q l\rangle}=\frac{\langle i j\rangle}{\langle q i\rangle\langle q j\rangle}
$$

we see that eqs. (C.13) and (C.15) are indeed the same.

\section{Integrals}

In this appendix we define our notation, and collect explicit expressions (through $\mathcal{O}\left(\epsilon^{0}\right)$ ) for the loop momentum integrals used in the paper. The one-loop scalar $m$-point integral in $D$ dimensions is defined by

$$
\begin{aligned}
\mathcal{I}_{m}^{K_{1} K_{2} \cdots K_{m}} & \equiv \int \frac{d^{D} L}{(2 \pi)^{D}} \frac{1}{L^{2}\left(L-K_{1}\right)^{2}\left(L-K_{1}-K_{2}\right)^{2} \ldots\left(L-\sum_{i=1}^{m-1} K_{i}\right)^{2}} \\
& \equiv \int \frac{d^{4} \ell}{(2 \pi)^{4}} \frac{d^{-2 \epsilon} \mu}{(2 \pi)^{-2 \epsilon}} \frac{1}{\left(\ell^{2}-\mu^{2}\right)\left(\left(\ell-K_{1}\right)^{2}-\mu^{2}\right) \ldots\left(\left(\ell-\sum_{i=1}^{m-1} K_{i}\right)^{2}-\mu^{2}\right)},
\end{aligned}
$$

where $K_{1}, K_{2}, \ldots, K_{m}$ are the (four-dimensional) external momenta for the integral, and $L$ is the

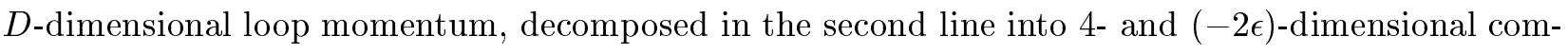
ponents, $L=\ell+\mu$. In general, the $K_{i}$ may be either individual massless external momenta $k_{j}$ for the amplitude under consideration, or else sums of such external momenta. To simplify the notation, in the former case we will replace $K_{i}$ in the argument of $\mathcal{I}_{m}$ simply by the appropriate integer index $j$; in the latter case we will often replace it by the set of integers entering the momentum sum, enclosed in parentheses. For example, one of the box integrals encountered in six-point amplitudes contains two diagonally-opposite massive external legs, with masses $s_{23}$ and $s_{56}$, and is given by

$$
\mathcal{I}_{4}^{1(23) 4(56)} \equiv \int \frac{d^{D} L}{(2 \pi)^{D}} \frac{1}{L^{2}\left(L-k_{1}\right)^{2}\left(L-k_{1}-k_{2}-k_{3}\right)^{2}\left(L+k_{5}+k_{6}\right)^{2}\left(L+k_{6}\right)^{2}} .
$$

Integrals where an additional factor of $\left(\mu^{2}\right)^{r} \equiv \mu^{2 r}$ has been inserted into the loop integrand are denoted by

$$
\mathcal{I}_{m}^{K_{1} K_{2} \cdots K_{m}}\left[\mu^{2 r}\right] \equiv \int \frac{d^{4} \ell}{(2 \pi)^{4}} \frac{d^{-2 \epsilon} \mu}{(2 \pi)^{-2 \epsilon}} \frac{\mu^{2 r}}{\left(\ell^{2}-\mu^{2}\right)\left(\left(\ell-K_{1}\right)^{2}-\mu^{2}\right) \cdots\left(\left(\ell-\sum_{i=1}^{m-1} K_{i}\right)^{2}-\mu^{2}\right)},
$$

These integrals can be expressed, via eq. (3.9), in terms of the integrals $\mathcal{I}_{m}^{D=4+2 r-2 \epsilon}$, that is, $\mathcal{I}_{m}$ in eq. (D.1) with $D$ replaced by $D+2 r$.

The $N=4$ super-Yang-Mills and $N=8$ supergravity amplitudes contain scalar box integrals with one and two external masses. The two-mass box integral with two diagonally-opposite massive legs (see fig. 1), evaluated in $D=4-2 \epsilon$, is [40]

$$
\begin{aligned}
\mathcal{I}_{4}^{a K_{1} b K_{2}} & =i \frac{c_{\Gamma}}{S_{1 a} S_{1 b}-K_{1}^{2} K_{2}^{2}}\left\{\frac{2}{\epsilon^{2}}\left[\left(-S_{1 a}\right)^{-\epsilon}+\left(-S_{1 b}\right)^{-\epsilon}-\left(-K_{1}^{2}\right)^{-\epsilon}-\left(-K_{2}^{2}\right)^{-\epsilon}\right]\right. \\
& -2 \mathrm{Li}_{2}\left(1-\frac{K_{1}^{2}}{S_{1 a}}\right)-2 \mathrm{Li}_{2}\left(1-\frac{K_{1}^{2}}{S_{1 b}}\right)-2 \mathrm{Li}_{2}\left(1-\frac{K_{2}^{2}}{S_{1 a}}\right)-2 \mathrm{Li}_{2}\left(1-\frac{K_{2}^{2}}{S_{1 b}}\right) \\
& \left.+2 \mathrm{Li}_{2}\left(1-\frac{K_{1}^{2} K_{2}^{2}}{S_{1 a} S_{1 b}}\right)-\ln ^{2}\left(\frac{S_{1 a}}{S_{1 b}}\right)\right\}+\mathcal{O}(\epsilon),
\end{aligned}
$$


where $S_{1 a}=\left(K_{1}+k_{a}\right)^{2}, S_{1 b}=\left(K_{1}+k_{b}\right)^{2}$, and

$$
c_{\Gamma}=\frac{1}{(4 \pi)^{2-\epsilon}} \frac{\Gamma(1+\epsilon) \Gamma^{2}(1-\epsilon)}{\Gamma(1-2 \epsilon)} .
$$

One-mass box integrals also appear in the amplitudes, corresponding to the case where $K_{1}$ reduces to a single external momentum. They are also given by eq. (D.4) - one may set $K_{1}^{2}=0$ after dropping the $\left(-K_{1}^{2}\right)^{-\epsilon}$ term. (One may similarly set $K_{1}^{2}=K_{2}^{2}=0$ to obtain the box integral with no external masses encountered in the four-point amplitudes.)

For the all-plus gauge amplitudes (3.7) and gravity amplitudes (4.12), (4.20) and (4.25), only the ultraviolet-singular parts $\left(1 / \epsilon\right.$ poles) of the higher-dimensional integrals $\mathcal{I}_{m}^{D=4+2 r-2 \epsilon}$ contribute in the four-dimensional limit $\epsilon \rightarrow 0$, due to the overall prefactor of $\epsilon$ in eq. (3.9). Such terms are given by elementary integrals of polynomials in the Feynman parameters.

In the gauge theory case, the only divergent integrals that appear are $D=8-2 \epsilon$ box integrals and $D=10-2 \epsilon$ pentagon integrals. The box integral is

$$
\begin{aligned}
\mathcal{I}_{4}\left[\mu^{4}\right] & =-\epsilon(1-\epsilon)(4 \pi)^{2} \mathcal{I}_{4}^{D=8-2 \epsilon} \\
& =-i \epsilon(1-\epsilon) \frac{\Gamma(\epsilon)}{(4 \pi)^{2-\epsilon}} \int d^{4} a_{i} \delta\left(1-\sum_{i} a_{i}\right)\left(-s_{12} a_{1} a_{3}-s_{23} a_{2} a_{4}+\cdots\right)^{-\epsilon} \\
& =-\frac{i}{(4 \pi)^{2}} \frac{1}{6}+\mathcal{O}(\epsilon) .
\end{aligned}
$$

Since $\mathcal{I}_{4}^{D=8-2 \epsilon}$ is dimensionless, the pole in $\epsilon$ does not depend on the particular kinematic configuration, so we have suppressed the labels describing the kinematics. (External masses would contribute to the ' $+\cdots$ ' terms in eq. (D.6).) Similarly, the pentagon integral that appears is

$$
\begin{aligned}
\mathcal{I}_{5}\left[\mu^{6}\right] & =-\epsilon(1-\epsilon)(2-\epsilon)(4 \pi)^{3} \mathcal{I}_{5}^{D=10-2 \epsilon} \\
& =i \epsilon(1-\epsilon)(2-\epsilon) \frac{\Gamma(\epsilon)}{(4 \pi)^{2-\epsilon}} \int d^{5} a_{i} \delta\left(1-\sum_{i} a_{i}\right)\left(-s_{45} a_{4} a_{1}+\text { cyclic }+\cdots\right)^{-\epsilon} \\
& =\frac{i}{(4 \pi)^{2}} \frac{1}{12}+\mathcal{O}(\epsilon),
\end{aligned}
$$

where the leading term again does not depend on the kinematic configuration. The hexagon integral in eq. (3.7) is

$$
\mathcal{I}_{6}\left[\mu^{6}\right]=-\epsilon(1-\epsilon)(2-\epsilon)(4 \pi)^{3} \mathcal{I}_{6}^{D=10-2 \epsilon}=\mathcal{O}(\epsilon),
$$

since $\mathcal{I}_{6}^{D=10-2 \epsilon}$ is finite.

In the all-plus gravity amplitudes, the required higher-dimensional integrals are dimensionful, and therefore depend on the kinematics. We give here the box and pentagon integrals with the maximum required number of external masses; those with fewer masses can be obtained by setting 
masses to zero. The two-mass box integral that appears in the amplitudes is

$$
\begin{aligned}
& \mathcal{I}_{4}^{a K_{1} b K_{2}}\left[\mu^{8}\right]=-\epsilon(1-\epsilon)(2-\epsilon)(3-\epsilon)(4 \pi)^{4} \mathcal{I}_{4}^{a K_{1} b K_{2}, D=12-2 \epsilon} \\
&=-i \epsilon(1-\epsilon)(2-\epsilon)(3-\epsilon) \frac{\Gamma(-2+\epsilon)}{(4 \pi)^{2-\epsilon}} \\
& \quad \times \int d^{4} a_{i} \delta\left(1-\sum_{i} a_{i}\right)\left(-S_{1 a} a_{1} a_{3}-S_{1 b} a_{2} a_{4}-K_{1}^{2} a_{2} a_{3}-K_{2}^{2} a_{4} a_{1}\right)^{2-\epsilon} \\
&=-\frac{i}{(4 \pi)^{2}} \frac{1}{840}\left[2 S_{1 a}^{2}+2 S_{1 b}^{2}+2\left(K_{1}^{2}\right)^{2}+2\left(K_{2}^{2}\right)^{2}+S_{1 a} S_{1 b}+K_{1}^{2} K_{2}^{2}\right. \\
&\left.\quad+2\left(S_{1 a}+S_{1 b}\right)\left(K_{1}^{2}+K_{2}^{2}\right)\right]+\mathcal{O}(\epsilon),
\end{aligned}
$$

where the kinematic configuration is depicted in fig. 1.

The one-mass pentagon integral that appears in the six-point all-plus gravity amplitude is

$$
\begin{aligned}
& \mathcal{I}_{5}^{1234(56)}\left[\mu^{10}\right]=-\epsilon(1-\epsilon) \cdots(4-\epsilon)(4 \pi)^{5} \mathcal{I}_{5}^{1234(56), D=14-2 \epsilon} \\
&= i \epsilon(1-\epsilon) \cdots(4-\epsilon) \frac{\Gamma(-2+\epsilon)}{(4 \pi)^{2-\epsilon}} \int d^{5} a_{i} \delta\left(1-\sum_{i} a_{i}\right) \\
& \quad \times\left(-s_{12} a_{1} a_{3}-s_{23} a_{2} a_{4}-s_{34} a_{3} a_{5}-t_{456} a_{4} a_{1}-t_{561} a_{5} a_{2}-s_{56} a_{5} a_{1}\right)^{2-\epsilon} \\
&= \frac{i}{(4 \pi)^{2}} \frac{1}{1680}\left[2 s_{12}^{2}+2 s_{23}^{2}+2 s_{34}^{2}+2 s_{56}^{2}+2 t_{456}^{2}+2 t_{561}^{2}+2 s_{12} s_{34}+2 s_{12} s_{56}+2 s_{34} s_{56}\right. \\
& \quad+2 s_{12} t_{456}+2 s_{34} t_{561}+2\left(s_{23}+s_{56}\right)\left(t_{456}+t_{561}\right) \\
&\left.\quad+s_{12} s_{23}+s_{23} s_{34}+s_{23} s_{56}+s_{12} t_{561}+s_{34} t_{456}+t_{456} t_{561}\right]+\mathcal{O}(\epsilon) .
\end{aligned}
$$

To obtain the massless pentagon integral appearing in the five-point amplitude from eq. (D.10), simply replace $k_{5}+k_{6}$ by $k_{5}$, i.e., $s_{56} \rightarrow 0, t_{456} \rightarrow s_{45}$, and $t_{561} \rightarrow s_{51}$.

\section{References}

[1] H. Kawai, D.C. Lewellen and S.-H.H. Tye, Nucl. Phys. B269:1 (1986).

[2] M.T. Grisaru, H.N. Pendleton and P. van Nieuwenhuizen, Phys. Rev. D15:996 (1977);

M.T. Grisaru and H.N. Pendleton, Nucl. Phys. B124:81 (1977);

S.J. Parke and T. Taylor, Phys. Lett. 157B:81 (1985).

[3] S.J. Parke and T.R. Taylor, Phys. Rev. Lett. 56:2459 (1986).

[4] F.A. Berends, W.T. Giele and H. Kuijf, Phys. Lett. 211B:91 (1988).

[5] S. Weinberg, Phys. Lett. 9:357 (1964); Phys. Rev. 140:B516 (1965).

[6] Z. Bern, L. Dixon, D.C. Dunbar, M. Perelstein and J.S. Rozowsky, Nucl. Phys. B530:401 (1998) [hep-th/9802162].

[7] M.B. Green, J.H. Schwarz and L. Brink, Nucl. Phys. B198:474 (1982). 
[8] M.B. Green, J.H. Schwarz and E. Witten, Superstring Theory (Cambridge University Press, 1987).

[9] P.S. Howe, K.S. Stelle and P.K. Townsend, Nucl. Phys. B236:125 (1984);

P.S. Howe and K.S. Stelle, Phys. Lett. 137B:175 (1984), Int. J. Mod. Phys. A4:1871 (1989).

[10] R.E. Kallosh, Phys. Lett. 99B:122 (1981);

P.S. Howe, K.S. Stelle amd P.K. Townsend, Nucl. Phys. B191:445 (1981);

N. Marcus and A. Sagnotti, Nucl. Phys. B256:77 (1985).

[11] Z. Bern, L. Dixon, D.C. Dunbar and D.A. Kosower, Nucl. Phys. B425:217 (1994) [hep$\mathrm{ph} / 9403226]$.

[12] Z. Bern, L. Dixon and D.A. Kosower, in Proceedings of Strings 1993, eds. M.B. Halpern, A. Sevrin and G. Rivlis (World Scientific, Singapore, 1994) [hep-th/9311026];

Z. Bern, G. Chalmers, L. Dixon and D.A. Kosower, Phys. Rev. Lett. 72:2134 (1994) [hep$\mathrm{ph} / 9312333]$.

[13] G.D. Mahlon, Phys. Rev. D49:2197 (1994) [hep-ph/9311213]; Phys. Rev. D49:4438 (1994) [hep$\mathrm{ph} / 9312276]$.

[14] Z. Bern, L. Dixon, M. Perelstein and J.S. Rozowsky, preprint hep-th/9809160, to appear in Phys. Lett. B.

[15] Z. Bern, L. Dixon, D.C. Dunbar and D.A. Kosower, Phys. Lett. 394B:105 (1997) [hepth/9611127].

[16] Z. Bern, L. Dixon and D.A. Kosower, Ann. Rev. Nucl. Part. Sci. 46:109 (1996) [hep-ph/9602280].

[17] J.F. Plebański, J. Math. Phys. 16:2395 (1975);

M.J. Duff, in Proceedings of 1979 Supergravity Workshop, eds. P. Van Nieuwenhuizen and D.Z. Freedman (North Holland, 1979);

J.F. Plebański and M. Przanowski, Phys. Lett. A212:22 (1996) [hep-th/9605233];

A. Jevicki, M. Mihailescu and J.P. Nunes, e-print hep-th/9804206.

[18] C.N. Yang, Phys. Rev. Lett. 38:1377 (1977);

S. Donaldson, Proc. Lond. Math. Soc. 50:1 (1985);

A.N. Leznov, Theor. Math. Phys. 73:1233 (1988);

A.N. Leznov and M.A. Mukhtarov, J. Math. Phys. 28:2574 (1987);

V.P. Nair and J. Schiff, Phys. Lett. B246:423 (1990); Nucl. Phys. B371:329 (1992);

A. Parkes, Phys. Lett. B286:265 (1992) [hep-th/9203074];

W. Siegel, Phys. Rev. D46:R3235 (1992).

[19] M.J. Duff and C.J. Isham, Nucl. Phys. B162:271 (1980);

W.A. Bardeen, Prog. Theor. Phys. Suppl. 123:1 (1996);

K.G. Selivanov, preprint hep-ph/9604206;

A.A. Rosly and K.G. Selivanov, Phys. Lett. B399:135 (1997) [hep-th/9611101]. 
[20] D. Cangemi, Nucl. Phys. B484:521 (1997) [hep-th/9605208]; Int. J. Mod. Phys. A12:1215 (1997) [hep-th/9610021].

[21] A.A. Rosly and K.G. Selivanov, preprint hep-th/9710196.

[22] G. Chalmers and W. Siegel, Phys. Rev. D54:7628 (1996) [hep-th/9606061].

[23] G. Chalmers and W. Siegel, unpublished.

[24] S. Gubser and I. Klebanov, Phys. Lett. B413:41 (1997) [hep-th/9708005];

J. Maldacena, Adv. Theor. Math. Phys. 2:231 (1998) [hep-th/9711200];

S. Gubser, I. Klebanov and A. Polyakov, Phys. Lett. B428:105 (1998) [hep-th/9802109];

G. Horowitz and H. Ooguri, Phys. Rev. Lett. 80:4116 (1998) [hep-th/9802116];

E. Witten, Adv. Theor. Math. Phys. 2:253 (1998) [hep-th/9802150].

[25] L. Dixon, J. Harvey, C. Vafa and E. Witten, Nucl. Phys. B261:678 (1985); Nucl. Phys. B274:285 (1986).

[26] S. Kachru and E. Silverstein, Phys. Rev. Lett. 80:4855 (1998) [hep-th/9802183];

A. Lawrence, N. Nekrasov and C. Vafa, hep-th/9803015;

M. Bershadsky and A. Johansen, hep-th/9803249;

M. Bershadsky, Z. Kakushadze and C. Vafa, Nucl. Phys. B523:59 (1998) [hep-th/9803076].

[27] J.E. Paton and H.-M. Chan, Nucl. Phys. B10:516 (1969);

F.A. Berends and W.T. Giele, Nucl. Phys. B294:700 (1987);

D.A. Kosower, B.-H. Lee and V.P. Nair, Phys. Lett. 201B:85 (1988).

[28] M. Mangano, S. Parke, and Z. Xu, Nucl. Phys. B298:653 (1988).

[29] P. De Causmaecker, R. Gastmans, W. Troost and T.T. Wu, Phys. Lett. 105B:215 (1981), Nucl. Phys. B206:53 (1982);

R. Kleiss and W.J. Stirling, Nucl. Phys. B262:235 (1985);

J.F. Gunion and Z. Kunszt, Phys. Lett. 161B:333 (1985);

Z. Xu, D.-H. Zhang and L. Chang, Nucl. Phys. B291:392 (1987).

[30] F.A. Berends and W.T. Giele, Nucl. Phys. B313:595 (1989).

[31] M. Mangano and S.J. Parke, Phys. Rep. 200:301 (1991).

[32] L. Dixon, in Proceedings of Theoretical Advanced Study Institute in Elementary Particle Physics (TASI 95), ed. D.E. Soper [hep-ph/9601359].

[33] Z. Bern, L. Dixon and D.A. Kosower, Nucl. Phys. B437:259 (1995) [hep-ph/9409393].

[34] Z. Bern, L. Dixon, D.C. Dunbar and D.A. Kosower, Nucl. Phys. B435:59 (1995) [hepph/9409265]. 
[35] L.D. Landau, Nucl. Phys. 13:181 (1959);

S. Mandelstam, Phys. Rev. 112:1344 (1958), 115:1741 (1959);

R.E. Cutkosky, J. Math. Phys. 1:429 (1960).

[36] Z. Bern and A.G. Morgan, Nucl. Phys. B467:479 (1996) [hep-ph/9511336];

J.S. Rozowsky, preprint hep-ph/9709423.

[37] F.A. Berends and W.T. Giele, Nucl. Phys. B306:759 (1988).

[38] D.A. Kosower, Nucl. Phys. B335:23 (1990).

[39] Z. Bern and D.A. Kosower, Nucl. Phys. B362:389 (1991).

[40] Z. Bern, L. Dixon and D.A. Kosower, Nucl. Phys. B412:751 (1994) [hep-ph/9306240]; Phys. Lett. B302:299 (1993), erratum B318:649 (1993) [hep-ph/9212308].

[41] Z. Bern and G. Chalmers, Nucl. Phys. B447:465 (1995) [hep-ph/9503236].

[42] Z. Bern and D.A. Kosower, Nucl. Phys. B379:451 (1992). Z. Kunszt, A. Signer and Z. Trócsányi, Nucl. Phys. B411:397 (1994) [hep-ph/9305239].

[43] Z. Bern, L. Dixon and D.A. Kosower, Phys. Rev. Lett. 70:2677 (1993) [hep-ph/9302280];

Z. Kunszt, A. Signer and Z. Trócsányi, Phys. Lett. B336:529 (1994) [hep-ph/9405386].

[44] G. 't Hooft and M. Veltman, Nucl. Phys. B44:189 (1972).

[45] S.J. Gates, M.T. Grisaru, M. Roček and W. Siegel, Superspace (Benjamin/Cummings, 1983), pages $390-391$.

[46] Z. Bern, in Proceedings of Theoretical Advanced Study Institute in High Energy Physics (TASI 92), eds. J. Harvey and J. Polchinski (World Scientific, 1993) [hep-ph/9304249].

[47] Z. Bern and A. Morgan, Phys. Rev. D49:6155 (1994) [hep-ph/9312218].

[48] L.M. Brown and R.P. Feynman, Phys. Rev. 85:231 (1952);

G. Passarino and M. Veltman, Nucl. Phys. B160:151 (1979);

G. 't Hooft and M. Veltman, Nucl. Phys. B153:365 (1979).

[49] M.T. Grisaru and J. Zak, Phys. Lett. 90B:237 (1980).

[50] D.C. Dunbar and P.S. Norridge, Nucl. Phys. B433:181 (1995) [hep-th/9408014].

[51] Z. Bern, L. Dixon, D.A. Kosower and S. Weinzierl, Nucl. Phys. B489:3 (1997) [hep-ph/9610370];

Z. Bern, L. Dixon and D.A. Kosower, Nucl. Phys. B513:3 (1998) [hep-ph/9708239].

[52] Z. Bern, J.S. Rozowsky and B. Yan, Phys. Lett. B401:273 (1997) [hep-ph/9702424].

[53] W. Siegel, Phys. Lett. 84B:193 (1979);

D.M. Capper, D.R.T. Jones and P. van Nieuwenhuizen, Nucl. Phys. B167:479 (1980). 
[54] W.L. van Neerven, Nucl. Phys. B268:453 (1986).

[55] R. Kleiss and H. Kuijf, Nucl. Phys. B312:616 (1989);

F.A. Berends, W.T. Giele and H. Kuijf, Nucl. Phys. B333:120 (1990).

[56] Z. Bern, D.C. Dunbar and T. Shimada, Phys. Lett. B312:277 (1993) [hep-th/9307001].

[57] D.C. Dunbar, private communication.

[58] B.S. DeWitt, Phys. Rev. 162:1239 (1967);

M. Veltman, in Les Houches 1975, Proceedings, Methods In Field Theory (Amsterdam 1976);

S. Sannan, Phys. Rev. D34:1749 (1986).

[59] D.C. Dunbar and P.S. Norridge, Class. Quant. Grav. 14:351 (1997) [hep-th/9512084].

[60] W. Siegel, Phys. Rev. D47:2504 (1993) [hep-th/9207043].

[61] Z. Bern and A.K. Grant, in preparation.

[62] J.-L. Gervais and A. Neveu, Nucl. Phys. B46:381 (1972). 